University of Louisville

ThinkIR: The University of Louisville's Institutional Repository

$12-2010$

Daily emotional experiences, stress, and PTSD symptoms among

midlife women exposed to intimate partner violence : an

experience sampling study.

Rebecca Ann Weigel
University of Louisville

Follow this and additional works at: https://ir.library.louisville.edu/etd

Recommended Citation

Weigel, Rebecca Ann, "Daily emotional experiences, stress, and PTSD symptoms among midlife women exposed to intimate partner violence : an experience sampling study." (2010). Electronic Theses and Dissertations. Paper 1544.

https://doi.org/10.18297/etd/1544

This Doctoral Dissertation is brought to you for free and open access by ThinkIR: The University of Louisville's Institutional Repository. It has been accepted for inclusion in Electronic Theses and Dissertations by an authorized administrator of ThinkIR: The University of Louisville's Institutional Repository. This title appears here courtesy of the author, who has retained all other copyrights. For more information, please contact thinkir@louisville.edu. 


\title{
DAILY EMOTIONAL EXPERIENCES, STRESS, AND PTSD SYMPTOMS AMONG MIDLIFE WOMEN EXPOSED TO INTIMATE PARTNER VIOLENCE: \\ AN EXPERIENCE SAMPLING STUDY
}

\author{
By \\ Rebecca Ann Weigel \\ B.S., Centre College, 2002 \\ M.A., University of Louisville, 2005

\begin{abstract}
A Dissertation
Submitted to the Faculty of the

Graduate School of the University of Louisville

In Partial Fulfillment of the Requirements

For the Degree of
\end{abstract}

Doctor of Philosophy

Department of

Psychological and Brain Sciences

University of Louisville

Louisville, Kentucky

December 2010 
Copyright 2010 by Rebecca A. Weigel

All rights reserved 
DAILY EMOTIONAL EXPERIENCES, STRESS, AND PTSD SYMPTOMS AMONG MIDLIFE WOMEN EXPOSED TO INTIMATE PARTNER VIOLENCE: AN EXPERIENCE SAMPLING STUDY

\author{
By \\ Rebecca A. Weigel \\ B.S., Centre College, 2002 \\ M.A., University of Louisville, 2005 \\ A Dissertation Approved on
}

September 28,2010

by the following Dissertation Committee:

Dissertation Director 


\section{DEDICATION}

This work is dedicated to the courageous women who came forth to share their experiences and, by their willingness to do so, made this research possible; to my parents and my family, who have loved, supported, and provided me with countless opportunities; and to my spouse, Kyle, and daughter Alice, who believed in me, listened to me, and kept me going through the most trying of times. 


\section{ABSTRACT \\ DAILY EMOTIONAL EXPERIENCES, STRESS, AND PTSD SYMPTOMS AMONG MIDLIFE WOMEN EXPOSED TO INTIMATE PARTNER VIOLENCE: AN EXPERIENCE SAMPLING STUDY}

\section{Rebecca A. Weigel}

December 16, 2010

Described as important long-term consequences of trauma exposure, disruptions in emotional processes are regarded as central features of posttraumatic stress disorder (PTSD); however, the nature of these posttraumatic emotional disruptions remains poorly understood. The present study attempted to further understanding of these emotional disruptions by using experience sampling methodologies and electronic diaries to assess experiences of emotion, stress, and PTSD symptoms as they occurred in the day-to-day lives of midlife women exposed to intimate partner violence. Three sets of hypotheses were advanced and were tested using multilevel modeling approaches, with results demonstrating partial support for hypotheses overall. First, although past-month PTSD symptom severity was positively associated with daily negative emotion as expected, past-month PTSD symptom severity was, unexpectedly, not associated with positive emotion. Second, as expected, past-month PTSD symptom severity moderated withinpersons associations between daily stress and daily negative emotional experiences, such that women with greater PTSD symptom severity exhibited greater negative emotion on stressful days; however, contrary to expectations, no effect of past-month PTSD symptom severity was found for associations between stress and daily positive emotion. 
Third, while expected within-day positive associations between daily reexperiencing symptoms and daily negative emotion were not found, temporal associations emerged between emotional numbing symptoms, daily reexperiencing symptoms, and negative emotion such that emotional numbing symptoms on a given day were predicted by greater reexperiencing symptoms and lower negative emotion on the preceding day. In addition to expanding the existing literature on PTSD and emotion in general, the present study also offers a guide for future studies seeking to advance understanding of emotional processes in PTSD through the assessment of naturally occurring emotion and provides insights as to how psychological treatments aimed at improving emotional functioning among trauma-exposed individuals may be enhanced. 


\section{TABLE OF CONTENTS}

PAGE

DEDICATION .......................................................................... ii

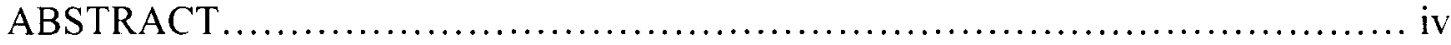

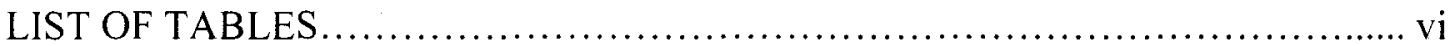

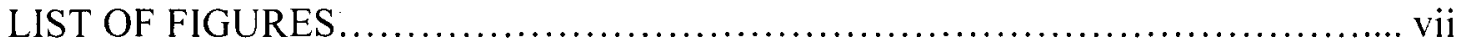

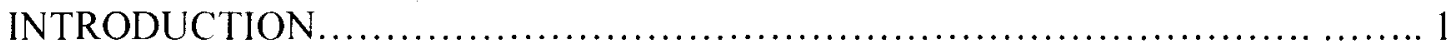

Defining Emotion .......................................................... 1

Emotional Processes in Posttraumatic Stress Disorder.............................. 2

Theoretical Explanations of Disrupted Emotional Processes in PTSD ................ 6

Empirical Investigations of Emotion and PTSD ................................ 9

Advancing Understanding of Emotional Processes in PTSD ..................... 16

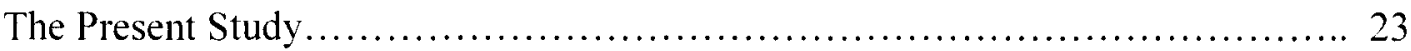

Statistical Power and Sample Size Considerations................................ 33

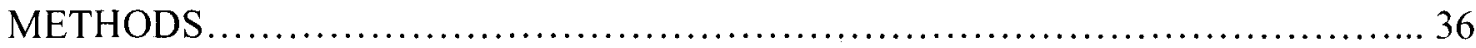

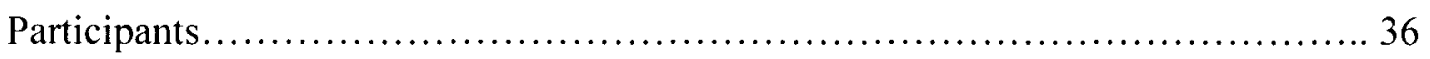

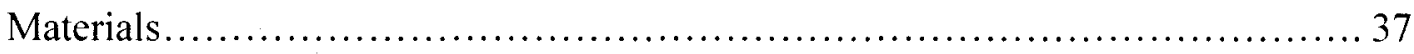

Measures of Between-Persons Factors........................................... 38

Measures of Within-Persons Factors: Daily Experiences...................... 41

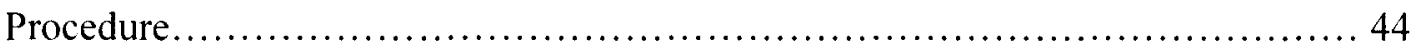

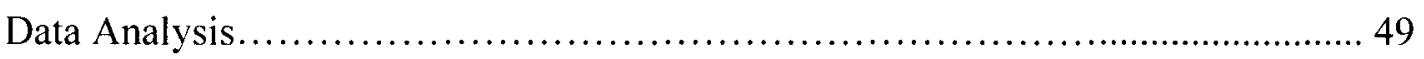

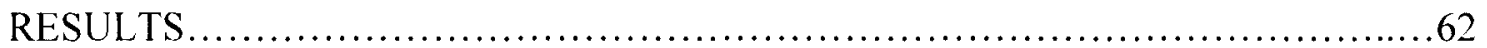


Compliance with Experience Sampling Procedures..............................62

Participant Characteristics................................................67

Aim 1: Characteristics of Daily Emotional Experiences.........................69

Aim 1: Characteristics of Daily PTSD Symptoms and Experiences of Stress.......... 73

Statistical Tests of Hypotheses............................................ 76

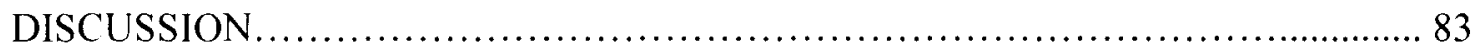

Hypotheses Set 1: Is PTSD Related to Women's Daily Experiences of

Emotion? 84

Hypotheses Set 2: Does PTSD Moderate Associations Between Stress and Daily Emotion? 91

Hypotheses Set 3: Are Daily PTSD Symptoms associated with Daily Emotion Levels? 95

Limitations, Special Considerations, and Future Directions. 101

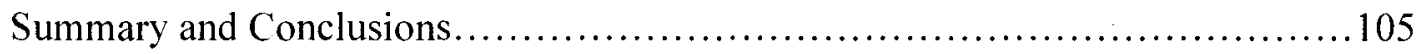

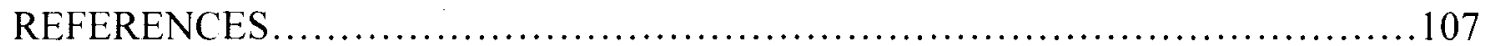

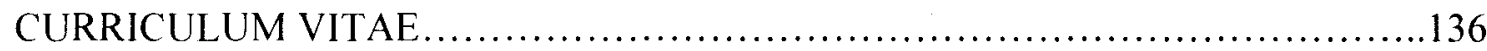




\section{LIST OF TABLES}

TABLE

PAGE

1. Sample Characteristics...................................................... 127

2. Means (SD) of Daily Experiences Averaged Across Women and Sampling

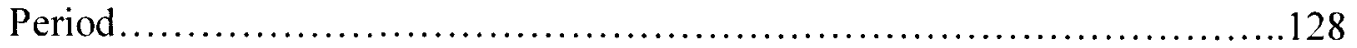

3. Social Context of Daily Emotional Experiences............................... 129

4. Daily Emotion Modeled as a Function of Neuroticism and Past-month PTSD

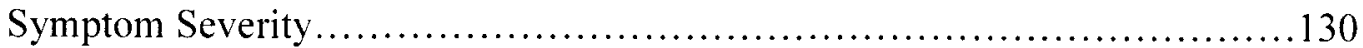

5. Daily Emotion Modeled as a Function of Neuroticism, Past-month PTSD Symptom Severity, and Daily Stress............................................ 131

6. Daily Emotional Numbing Symptoms Modeled as a Function of Lagged Daily Reexperiencing Symptoms and Negative Emotion. 


\section{INTRODUCTION}

\section{Defining Emotion}

Emotion is a fundamental and necessary part of human life. Central to human functioning and well-being, emotions have broad implications for quality of life (e.g., Booth \& Pennebaker, 2000; Diener \& Lucas, 2000). Emotions serve as primary sources of motivation for behavior (e.g., Izard, 1977), and play important roles in interpersonal processes and functioning (e.g., Baumeister, Stillwell, \& Heatherton, 1994), stress and coping processes (e.g., Lazarus, 1991), and physical health (e.g., Leventhal \& PatrickMiller, 2000). While by most definitions, an emotion is said to be short in duration, it has been suggested that one is never without the experience of emotion as, at any given time, one is always experiencing some emotion to some degree (e.g., Izard, 1977).

Although the nomenclature used for defining and describing emotions and their related constructs continues as a point of debate within the literature in general, a unifying theme among most contemporary emotion theories is the emphasis on the function of emotions (Gross, 1999). Functional approaches to emotion emphasize their adaptive nature and view emotions as phenomena evolved to coordinate a diverse array of responses needed for survival (Cosmides \& Tooby, 2000). Implicit in this approach is the idea that emotions are differentiated on the basis of the adaptive functions they serve and manifest as discrete entities characterized by their specific patterns of physiological, behavioral/expressive, and experiential components (Izard \& Ackerman, 2000). 
Although contemporary theorists differ in their outline of "discrete" emotions, most

identify some subset or combination of the following in their classifications: interest; joy; surprise; sadness; anger; disgust; fear; shame; and guilt (e.g., Izard, 1977; Plutchik, 1980; Ekman, 1992; Oatley \& Johnson-Laird, 1987; Fridja, 1986).

A functional approach to emotion necessitates regulatory mechanisms in place to ensure that emotions promote appropriate or adaptive responses in the individual but prevent emotions from occurring inappropriately or at levels that hinder adaptive responses (Cichetti, Ackerman, \& Izard, 1995). For example, anger may be adaptive for survival in that it motivates defense and attack behaviors when under threat, but anger may also serve to thwart other adaptive responses, such as creating or maintaining social ties, when no actual threat is present (e.g., Chemtob, Novaco, Hamada, Gross, \& Smith, 1997). These self-regulatory strategies, collectively termed "emotion regulation", reflect a constellation of "processes by which individuals influence which emotions they have, when they have them. and how they experience and express these emotions" (Gross, 1998, p. 275). Thus, in the most basic sense, regulating emotional processes are viewed as either conscious or nonconscious efforts aimed at decreasing, increasing, or initiating emotions as changing circumstances render specific emotional responses more or less adaptive (Gross, 1999).

\section{Emotional Processes in Posttraumatic Stress Disorder}

The notion that regulating one's emotional processes is necessary for optimal functioning is not a concept unique to contemporary theory; indeed, ideas about how much control individuals should exert over their emotions are evident in writings throughout history (Gross, 1998). For example, Aristotle described the importance of 
moderating one's emotional responses but also noted that the lack of certain emotions under appropriate circumstances represented a "vice" rather than a "virtue" (Solomon, 2000). In a similar vein, the centrality of disrupted emotional processes to psychopathology has also long been recognized (Cichetti et al., 1995). For example, in his writings on schizophrenia, Bleuler (1924) noted that "affectivity ... assumes a prominent role in psychopathology generally, even in slight deviations, not only on account of its own morbid manifestations, but even more because in disturbances in any sphere, it is the affective mechanisms that first create the manifest symptom" (p. 117). More recently, it has been noted that the vast majority of psychological disorders in the Diagnostic and Statistical Manual of Mental Disorders - Fourth edition (DSM-IV; APA, 1994) contain at least one symptom reflecting some disruption in emotional processes or emotion regulation (Kring \& Werner, 2004).

The Nature of Posttraumatic Stress Disorder

Although formally classified as a disorder of anxiety, posttraumatic stress disorder (PTSD) differs from other anxiety disorders in that symptoms are tied to and follow the experience of particularly stressful, or traumatic, events (APA, 1994). Current diagnostic criteria, according to the $D S M-I V$ (APA, 1994), define a traumatic event as one that involves actual or threatened death, serious injury, or threat to one's physical integrity and require that an individual either witness or directly experience such an event. A diagnosis of PTSD is qualified by the presence of specific posttraumatic responses, or symptoms, for at least one month following exposure to a traumatic event. These symptoms are classified into three broad clusters characterized by persistent reexperiencing of the traumatic event (e.g., intrusive and distressing recollections of the 
event, intense psychological distress at exposure to internal or external cues that symbolize or resemble the traumatic event); avoidance of stimuli associated with the trauma (e.g., efforts to avoid thoughts, feelings, or conversations associated with the traumatic event); and hyperarousal (e.g., hypervigilance, difficulty falling or staying asleep) (APA, 1994).

The importance of intense emotion in the development of posttraumatic symptomatology is evident in the $D S M-I V$ as a subjective response of intense fear, helplessness, or horror to a potentially traumatic event is also required for a diagnosis of PTSD (APA, 1994). In addition, disruptions in emotional processes or emotion regulation are also consistently recognized as long-term consequences of trauma exposure (Litz, Orsillo, Kaloupek, \& Weathers, 2000). However, although described as central defining features of PTSD, it has been noted that the nature of these emotional disruptions in PTSD continues to be poorly understood (Litz et al., 2000; Litz \& Gray, 2002).

The Nature of Disrupted Emotional Processes in PTSD

As outlined in $D S M-I V$ diagnostic criteria, the emotional disruptions associated with PTSD take on seemingly paradoxical forms marked by "hyperemotionality", or the experience of intense negative emotions, on the one hand, and "hypoemotionality", or emotional numbing, on the other (Litz, 1992, p. 418-419). With respect to the experience of intense negative emotions, in addition to experiences of nonspecific intense "distress" upon being reminded of the trauma, the $D S M-I V$ also makes explicit reference to increased irritability and feelings of anger as a diagnostic criterion of PTSD and includes "painful guilt feelings" as a common feature of the disorder. Furthermore, the DSM-IV 
specifies an "associated constellation of symptoms... commonly seen with an interpersonal stressor (i.e., childhood sexual or physical abuse, domestic battering)" and includes among these associated symptoms "impaired affect modulation", "feelings of ineffectiveness", and "shame" (APA, 1994, p. 425).

In contrast, emotional numbing. referred to as "psychic numbing" or "emotional anesthesia" in the $D S M-I V$, is represented in the following three PTSD diagnostic criteria within the avoidance symptom cluster: "markedly diminished interest or participation in previously enjoyed activities"; "feeling detached or estranged from others"; and a "markedly reduced ability to feel emotions" or "a restricted range of affect" (APA, 1994, p. 425). Described as the lesser understood of the emotional disruptions associated with PTSD (e.g., Litz \& Gray, 2002), the concept of emotional numbing is not without debate in the literature. That is, many have noted the lack of definitional clarity inherent in $D S M-I V$ descriptions of numbing and have suggested that the very term emotional numbing may be construed as a disservice to individuals with PTSD because it does not accurately convey the "dimensionality of their emotions" (Litz \& Gray, 2002, p. 199). Furthermore, while factor analytic studies have supported numbing symptoms as representing a distinct phenomenon from avoidance (Foa, Riggs, \& Gershuny, 1995), others have suggested that the emotional numbing observed in PTSD may be more closely aligned with anhedonia associated with depression (Kashdan, Elhai, \& Frueh, 2006; Monson, Price, Rodriguez, Ripley, \& Warner, 2004). Indeed, a recent confirmatory factor analytic study of PTSD symptoms among women exposed to intimate partner violence revealed a four-factor model. Reexperiencing, avoidance, and hyperarousal symptoms comprised three factors. The fourth factor, which the authors 
labeled "dysphoria", consisted of DSM-IV emotional numbing symptoms (i.e., loss of interest, feelings of detachment, and restricted range of affect) as well as symptoms classified in DSM-IV under the hyperarousal cluster (i.e., sleep disturbance, irritability, and impaired concentration) (Krause, Kaltman, Goodman, \& Dutton, 2007), all symptoms overlapping with $D S M-I V$ symptoms of major depression.

\section{Theoretical Explanations of Disrupted Emotional Processes in PTSD}

Although many models derived from a variety of theoretical approaches have been advanced to explain posttraumatic sequelae, those proposed by Foa and colleagues (e.g., Foa \& Sketee, \& Rothbaum, 1989; Foa \& Rothbaum, 1998), Horowitz (1986), and Litz and colleagues (e.g., Litz, 1992; Litz \& Gray, 2002) are particularly informative with respect to explaining posttraumatic emotional processes more specifically. An underlying premise of these theories is that all emotional experiences, whether occurring in a traumatic context or not, are often "relived" after the actual emotional experience has occurred and that reliving the emotional experience entails actually reexperiencing the original emotion itself, termed by some as "emotional reexperiencing" (e.g., Foa \& Rothbaum, 1998, p. 72). Such theories propose that exposure to a traumatic event results in the creation of a trauma memory, or a representation of the event in memory which contains associated information about stimulus characteristics of the event as well as associated emotional and physiological responses. An important underlying assumption of these theories is that traumatic events are represented differently with respect to the density, complexity, strength, and meaning of such associations in the memories of trauma-exposed individuals who go on to develop PTSD compared to those who do not (Foa \& Rothbaum, 1998). 
With respect to the experience of negative emotion associated with PTSD specifically, it is proposed that, upon confrontation with a trauma-related stimulus, activation of associations within the trauma memory network gives rise to reexperiencing symptoms and thus, associated intense negative emotional responses (Foa et al., 1989; Litz \& Gray, 2002). Over time, as associations within the trauma network become more generalized, a wide variety of internal (i.e., psychological or physiological) and external cues become associated with the trauma memory network such that even weakly-related trauma stimuli lead to activation of the trauma network, and thus, reexeperiencing symptoms and associated negative emotional responses (Foa et al., 1989; Litz \& Gray, 2002). In line with this thinking, it has been noted that individuals with PTSD often experience trauma-related physiological sensations or intense emotions without direct awareness of being confronted with a trauma cue, termed by some as "affect without recollection“ (e.g., Ehlers \& Clark, 2000, p. 324).

With respect to emotional numbing, Horowitz (1986) has proposed that continual traumatic reexperiencing results in heightened arousal due to the associated emotional reexperiencing while reliving the trauma. Emotional numbing then follows reexperiencing and subsequent emotional arousal in a phasic fashion, such that individuals with PTSD experience periods of intense reexperiencing and subsequent emotional arousal, followed by periods of emotional numbing. In this way, numbing acts as a defense against the painful emotion associated with reexperiencing the traumatic event. According to Horowitz (1986), these cyclic processes of reexperiencing (i.e., intrusion) and numbing (i.e., denial) actually represent recovery processes, or attempts to resolve the trauma memory and incorporate it into existing cognitive schemas. Although 
Horowitz (1986) does not advance explanation for the development of PTSD specifically, some have described this shift between reexperiencing and numbing as a "vicious cycle" that actually prevents emotional processing of the event and that promotes the continued reexperiencing and experience of intense negative emotions that are at the core of PTSD (Foa et al.,1995, p. 119).

Expanding on these ideas, Litz and colleagues (Litz, 1992; Litz \& Gray, 2002) posit that the emotional disruptions observed in PTSD (i.e., both increases in negative emotions and emotional numbing) largely result from both increased accessibility to negative emotions and decreased accessibility to positive emotions as a result of frequent reexperiencing states, or activation of the trauma memory network and conditioned negative emotional responses. They propose that, in addition to the influence of reexperiencing states on accessibility to positive emotion, decreased positive emotion observed among individuals with PTSD also results from these individuals" "preferential allocation of attention to threat, and the arousal that this process promotes, [which] generally raises the threshold required to respond emotionally to pleasant stimuli in PTSD“ (p. 202). Importantly, however, they argue that the capacity to experience the full range of emotion available to individuals prior to trauma remains intact following trauma exposure, including among individuals who go on to develop PTSD (Litz \& Gray, 2002). In sum, although these theories differ somewhat in their explanations of posttraumatic sequelae, they share some important features. First, each of these theories highlights emotional experiences as central components of trauma memories. Second, each portrays the emotional disruptions observed in PTSD as processes driven primarily by reexperiencing symptoms, or the activation of trauma memory networks. 
Questionnaire-based Studies of Emotion in PTSD

Cross-sectional questionnaire studies have established consistent linkages between PTSD and negative emotions in general. Using both trait and state measures of emotion, these cross-sectional studies have demonstrated significant and positive associations between PTSD and the tendency to experience negative emotion in general as well as tendencies to experience specific negative emotions. For example, crosssectional associations between positive PTSD diagnostic status and the tendency to experience frequent negative emotion, referred to as neuroticism (Costa \& McCrae, 1992) or trait negative affectivity (Watson \& Clark, 1984), have been demonstrated among a variety of trauma-exposed individuals (Cox, MacPherson, Enns, \& McWilliams, 2004). Likewise, significant, positive cross-sectional associations between PTSD symptom severity and more specific trait emotion measures, such as trait anger (e.g., Orth \& Wieland, 2006), guilt (Leskela, Dieperink, \& Thuras, 2002) and shame (e.g., Street \& Arias, 2001; Leskela et al., 2002), have been consistently demonstrated among a variety of trauma-exposed individuals.

The increase in intense, specific negative emotional experiences associated with PTSD (i.e., "hyperemotionality") is also well-documented among studies using either state-based emotion measures or measures of trauma-related emotion. For example, among combat veterans, these studies have demonstrated associations between positive PTSD diagnostic status and high levels of self-reported subjective state anger (e.g., Chemtob et al., 1997; Johnson et al., 1997), positive associations between PTSD symptom severity and high levels guilt (e.g., Henning \& Frueh, 1997; Kubany et al., 
1995; Kubany et al., 1996) and associations between positive PTSD diagnostic status and high levels of self-reported state shame (e.g., Johnson et al., 1997). Similarly, these studies have demonstrated significant and positive cross-sectional associations between either PTSD diagnostic status or PTSD symptom severity and high levels of self-reported subjective anger (e.g., Riggs, Dancu, Gershuny, Greenburg, \& Foa, 1992; Zoellner, Goodwin, \& Foa, 2000), guilt (e.g., Resick, Nishith, Weaver, Astin, \& Feuer, 2002), and shame (e.g., Feiring, Taska, \& Chen, 2002) among female victims of sexual assault or abuse, as well as between PTSD symptom severity and self-reported subjective guilt among women exposed to domestic violence (e.g., Kubany et al., 1995; Kubany et al., 1996; Street, Gibson, \& Holohan, 2005).

In contrast, studies of emotional numbing are comparatively fewer, and many of these studies have been concerned with relationships of emotional numbing symptoms to other symptoms of PTSD rather than assessing specific emotional experiences. For example, cross-sectional studies have demonstrated significant associations between emotional numbing symptoms and hyperarousal symptoms, leading some to conclude that emotional numbing in PTSD may reflect an "emotional depletion" as a result of chronic hyperarousal (Litz et al., 1997; Flack, Litz, Hsieh, Kaloupek, \& Keane, 2000). Likewise, using prospective, longitudinal designs in which PTSD symptoms were measured within weeks of trauma exposure and at three and 12 months post-trauma, two studies found that emotional numbing symptoms at each time point were best predicted by antecedent hyperarousal symptoms at the previous assessment (Schell, Marshall, \& Jaycox, 2004; Marshall, Schell, Glynn, \& Shetty, 2006). Other studies of emotional numbing in PTSD have examined relationships of numbing symptoms to other broad 
aspects of emotional functioning. For example, among combat veterans, one study found no significant association between emotional numbing symptoms and alexythymia (i.e., defined as difficulty identifying or describing feelings) among veterans with PTSD (Monson et al., 2004), while another study found that self-reported fear of losing affective control was associated with other PTSD symptom clusters but not avoidance/emotional numbing (Price, Monson, Callahan, \& Rodriguez, 2006). In a similar vein, Roemer, Litz, Orsillo, and Wagner (2001) demonstrated that combat veterans with PTSD endorsed higher levels of intentionally "withholding" both positive and negative emotional responses compared to veterans without PTSD, suggesting that individuals may actually be exerting some voluntary control over the restricted emotion that is posited to occur with emotional numbing.

While collectively these studies are important in that they help to construct an overall characterization of the emotional disruptions associated with PTSD, and while the studies of emotional numbing in particular help to shed some light on potential relationships of emotional disruptions to specific symptoms, such studies prevent a finegrained examination of disrupted emotional processes in PTSD due to their reliance on more global measures of emotional experiences. Although of considerably fewer numbers, laboratory studies of emotional responses among individuals with PTSD offer improvements in this regard in that they allow the examination of momentary emotional responses to well-defined stimuli. These laboratory studies have examined emotional responses to trauma-related cues as well as non-trauma related neutral, negatively- and positively-valenced emotional stimuli among individuals with and without PTSD. Laboratory Studies of Emotional Responses in PTSD 


\section{Emotional responses to truuma cues. Although several psychophysiological}

studies have assessed autonomic nervous system arousal to trauma cues, one study also assessed subjective emotion responses to trauma-related stimuli among Vietnam combat veterans with PTSD and a control group of veterans without PTSD (Pitman, Orr, Forgue, de Jong, \& Claiborn, 1987). Veterans first wrote scripts of what they identified as their two most stressful combat experiences and scripts of a pre-combat traumatic experience which were then read by the experimenter and audiotaped. After listening to each of the scripts they had written, veterans rated their subjective discrete emotional responses (i.e., happiness, sadness, fear, surprise, anger, and disgust). Results indicated that in response to the combat script, veterans with PTSD reported significantly greater sadness, fear, anger, and disgust, and significantly less happiness compared to veterans without PTSD. In response to the pre-combat trauma script, veterans with PTSD reported significantly more sadness compared to veterans without PTSD.

Emotional responses to non trauma-specific emotional stimuli. Two studies examined subjective emotional responses to emotionally evocative images projected on slides or films. Amdur, Larsen, \& Liberzon (2000) examined emotional responses among male Vietnam veterans with combat-related PTSD, a group of control male combat veterans with no PTSD, and a group of control men with no combat experience. Men rated their subjective emotional responses (i.e., disgusted, calm, surprised, angry, afraid, ashamed, sad, and pleased) while viewing slides of emotionally evocative images categorized as "exciting" (e.g., sports scenes, nude women), "distressing" (e.g., snake, gun, mutilated bodies). and "unpleasant" (e.g., dead animals, starving child). Emotion ratings among groups were comparable across image type (i.e., exciting, distressing, 
unpleasant), but men with PTSD reported more intense anger, disgust, shame, sadness, and less intense fear, than did either control group with respect to a specific subset of particularly "evocative" images. However, as the authors did not specify in what ways these slides were more emotionally evocative, it seems possible that the higher negative emotion in response to some slides and not others could have been attributable to the greater trauma relevance of certain images (i.e., gun, mutilated bodies, starving children), such that the higher emotional responses were actually reflective of emotional reexperiencing to trauma cues.

Using a similar paradigm, Orsillo, Batten, Plumb, Luterek, and Roessner (2004) compared emotional responses among female sexual assault victims endorsing PTSD symptomatology and non-traumatized control women who described themselves as generally happy and well-adjusted. Women rated their subjective emotional responses on the dimensions of arousal and valence to films chosen to evoke emotional responses of amusement, sadness, fear, anger, and to a film clip of waves on a beach designed to elicit feelings of contentment. In addition, women provided written accounts of their emotional reactions to the films. Results indicated that, while women with PTSD reported more negative emotion in response to all films compared to control women, women with PTSD also reported more positive emotion in response to the films, but only those designed to elicit fear and anger, than did control women. With respect to the written emotional responses accounts, there were no significant differences in the number of positive emotion words used among women; however, women with PTSD used more negative emotion words in response to all film clips with the exception of the contentment film. 
Emotional responses to non-traumatic emotional stimuli following priming. In an attempt to examine whether exposure to trauma reminders influences subsequent emotional responses to non-traumatic emotional stimuli, two studies compared emotional responses to non trauma-related emotionally evocative images following exposure to neutral and traumatic priming conditions. Employing a sample of male Vietnam combat veterans with or without PTSD, Litz, Orsillo, Kaloupek, and Weathers (2000) examined subjective emotional responses to emotionally evocative photographs designed to elicit positive, negative, or neutral emotional responses. Veterans viewed these images following exposure to two experimental conditions, a neutral priming condition in which veterans viewed still photos of furniture and listened to piano music, and a trauma priming condition in which veterans viewed still images of Vietnam combat situations and listened to war zone sounds. No significant differences in emotional responses to positive, negative, or neutral photos between veterans with or without PTSD were found for either priming condition.

Likewise, employing a sample of female victims of sexual assault endorsing PTSD symptoms and a control group of nontraumatized women without PTSD, Wagner, Roemer, Orsillo, and Litz (2003) assessed subjective emotional responses rated on dimensions of arousal and valence to images on slides designed to evoke positive, neutral, and negative emotional responses following two priming conditions. In the trauma priming condition, traumatized women were instructed to write about their traumatic experience in detail, including sensory and emotional experiences, and listened to an audiotape of their account, while control women listened to an account of a traumatized woman matched for age and ethnicity. In the neutral priming condition, all 
women were instructed to write about neutral experiences in detail, such as how to brush one's teeth, and then listened to their account. Results indicated that, compared to control women, women with PTSD reported overall greater arousal, but not valence, to both positive and negative slides regardless of priming condition.

In summary, in contrast to the questionnaire-based studies of general self-reported emotion and PTSD cited above, the laboratory studies of emotional responses provide a somewhat different and more complicated picture of emotional processes among individuals with PTSD. That is, while Amdur et al. (2000) and Litz et al. (2000) found no differences in subjective emotional responses to non-traumatic emotional stimuli overall with respect to PTSD status among male combat veterans, Orsillo et al. (2004) found that women with PTSD reported more negative emotion to all emotional stimuli, and interestingly, more positive emotion to anger and fear stimuli, and Wagner et al. (2003) reported that women with PTSD reported more emotional arousal to all stimuli compared to women without PTSD. Moreover, none of the studies provided evidence for emotional numbing, or diminished emotional responses, to emotional stimuli either when comparing traumatized individuals with PTSD and nontraumatized controls (e.g., Orsillo et al., 2004) or when comparing traumatized individuals with PTSD and traumatized controls without PTSD (e.g., Amdur et al., 2000; Litz et al., 2000). Additionally, although Pitman et al. (1987) observed greater negative emotional responses to traumarelated stimuli among veterans with PTSD, exposure to trauma cues (i.e., trauma priming) in other studies did not appear to increase or decrease emotional responses to subsequent non-traumatic stimuli among individuals with PTSD. Thus, these studies call into question theoretical formulations positing negative emotional experiences in PTSD 
as processes driven by traumatic reexperiencing. Although the small number of studies prevent firm conclusions, when considered with the questionnaire-based studies cited above, these findings suggest more complex emotional processes among trauma-exposed individuals than perhaps previously considered. Thus, as many have noted (e.g., Orsillo et al., 2004; Litz \& Gray, 2002; Litz et al., 2000; Litz, 1992), the field is clearly in need of a better and more complete understanding of emotional processes in PTSD.

\section{Advancing Understanding of Emotional Processes in PTSD}

Individuals seeking treatment for PTSD report that the emotional disruptions they experience cause them significant impairment in their interpersonal relationships and in their day-to-day functioning (Cloitre, Koenen, Cohen, \& Han, 2002). Thus, while gaining a better understanding of emotional processes in PTSD is important in its own right, such an understanding takes on added significance when considering its implications for informing PTSD treatments. As just one illustration, is it appropriate to assume that treating the symptoms of PTSD, and particularly reducing reexperiencing symptoms, will result in improved emotional regulation among individuals with PTSD, as some have maintained (e.g., Litz, 1992; Litz et al., 2000; Litz \& Gray 2002)? Or, as others have suggested, should dysregulated emotional processes be addressed separately from the treatment of specific PTSD symptoms (e.g., Cloitre et al., 2002)? Based on the theoretical and empirical literature reviewed above, answering these questions requires, first, more accurate characterizations of emotional experiences among trauma-exposed individuals, and second, a better understanding of the relationship of emotional processes to specific symptoms. The existing questionnaire-based studies provide important information on the nature of emotional disruptions in PTSD, but they reveal less about 
the actual emotional experiences of individuals with PTSD. Moreover, because any process by nature involves an unfolding of events or experiences over time (e.g., Tennen, Affleck, Armeli, \& Carney, 2000), the exact nature of emotional processes in PTSD also cannot be revealed in either cross-sectional questionnaire studies of general or more global emotional experiences or in cross-sectional laboratory studies of specific emotional responses. Thus, an understanding the disrupted emotional processes in PTSD may be advanced more readily through the use of more ecologically valid methods and real-time assessment of emotional experiences and symptoms.

Naturalistic Study of Daily Experiences: Experience Sampling Methods

Experience sampling methods (ESM) were designed for gathering information on experiences within the context of everyday life and make use of daily diaries or diary reports of momentary experiences using signaling devices (Bolger, Davis, \& Rafaeli, 2003). The advantages conferred by ESM over traditional methodologies have particular relevance for the study of emotional processes in PTSD. First, assessments of daily experiences, such as emotional experiences, are carried out in individuals' natural environments (Stone \& Lichter-Kelly, 2006). Thus, the ecological validity afforded by these methods is particularly important given that gaining an understanding of emotional processes in PTSD may lead to improved PTSD treatments, and treatments are concerned primarily with improving individuals ${ }^{*}$ functioning in their daily lives. Second, because with ESM, experiences are measured in close proximity to their occurrence, biases associated with retrospective recall are reduced or eliminated (Stone \& Lichter-Kelly, 2006). Reducing recall bias in the study of emotional experiences and PTSD is especially important given evidence that severity of PTSD symptoms may produce bias 
in individuals ${ }^{*}$ retrospective reconstruction of events and experiences from memory (Southwick, Morgan, Nicolau, \& Charney, 1997). Third, because ESM provide repeated assessments of momentary experiences, patterns in different facets of emotional experiences may be examined. For example, it has been noted that the frequency and intensity of repeatedly measured emotions can provide very different information about emotional experiences (Diener, Larsen, Levine, \& Emmons, 1985; Schimmack \& Diener, 1997). That is, it may be the case that one individual experiences anger less frequently but very intensely and another individual experiences anger more frequently but to a milder degree. Thus, maintaining this distinction seems particularly important given the need for more accurate characterizations of actual emotional experiences in PTSD and the implications for treatment. Finally, because multiple reports are collected from each individual over time, ESM allow for identification of meaningful patterns in daily experiences and examination of temporal relationships among these daily experiences within individuals (Stone \& Lichter-Kelly, 2006). Thus, with respect to studying emotional disruptions in PTSD, utilizing such sampling methods allows for not only a richer and more accurate description of individuals' emotional experiences but also allows for testing theoretical propositions of disrupted emotional processes in PTSD via examination of temporal relationships between emotional experiences and specific PTSD symptoms. However, although experience sampling methods have been widely used to examine mood or emotional states among psychologically healthy individuals (Feldman Barrett \& Barrett, 2001) and individuals with other psychological disorders, such as affective and psychotic disorders (e.g., Peeters, Nicolson, Berkhof, Delespaul, \& de Vries, 2003; Myin-Germeys, Krabbendam, Delespaul, \& van Os, 2004), chronic pain 
disorders (e.g., Tennen, Affleck, \& Zatura, 2006; Nicassio, Moxham, Schuman, \& Geveritz, 2002), and substance use disorders (e.g., Simpson, Kivlahan, Bush, \& McFall, 2005), to date, very few studies have applied these methodologies to the study of PTSD and emotional experiences among trauma-exposed individuals.

Experience Sampling Studies of Emotional Experiences in Trauma-Exposed Individuals

Although their primary aim was to examine associations between PTSD and ambulatory blood pressure among male Vietnam combat veterans with and without PTSD, two studies also assessed veterans' emotional experiences via paper-and-pencil diaries (Muraoka, Carlson, \& Chemtob, 1998; Beckham et al., 2000). In the first study, veterans reported on each of three current negative mood states (i.e., stress, anxiety, and anger) approximately three times an hour during the course of one day (Muraoka et al., 1998), while in the second study, veterans completed ratings of positive (i.e., satisfied, calm) and negative (i.e., irritated, depressed) emotions approximately every 30 minutes over the waking hours of one day (Beckham et al., 2000). Results of both studies indicated that, compared to veterans without PTSD, veterans with PTSD exhibited significantly higher mean levels of all negative emotions measured (Muraoka et al., 1998; Beckham, 2000) and significantly lower mean levels of positive emotions (Beckham et al., 2000). Moreover, these associations held even after controlling for the effects of psychotropic medication use and history of major depression (Beckham et al., 2000).

While two other studies also employed samples of male Vietnam combat veterans to examine daily levels of positive and negative emotion, these studies used a somewhat different approach to evaluate the relationship of PTSD to emotional experiences. That is, one study investigated the effects of PTSD on daily "affect balance", a measure of 
emotional well-being operationalized by the authors as the arithmetical difference between positive and negative emotions experienced over a given day (Kashdan, Julian, Merritt, \& Uswatte, 2006, p. 566). Veterans reported on positive (i.e., happy, proud) and negative (i.e., anxious, frustrated) emotions once daily for 14 days via diaries completed at the end of each day. In this study, veterans with PTSD reported significantly greater daily negative emotion, significantly lower daily positive emotion, and evidenced significantly less daily affect balance compared to veterans without PTSD. The other study examined fluctuations in, or instability of, daily emotion (Kashdan, Uswatte, Steger, \& Julian, 2006). Veterans provided end-of-the-day dairy ratings for positive and negative emotions over the course of 14 days, and positive and negative emotion instability was derived by computing within-subject daily deviation scores from each veteran`s mean positive and negative emotion scores, respectively. Analyses revealed that, compared to veterans without PTSD, veterans with PTSD exhibited significantly greater instability of negative emotion (i.e., higher mean daily deviation scores), while a nonsignificant trend for greater instability of positive emotions among veterans with PTSD was observed.

While not assessing PTSD specifically, two studies have examined emotional experiences among individuals exposed to potentially traumatic events. In the first study, male and female college students endorsing various traumas (i.e., sexual or physical assault, motor vehicle accidents, fires, or natural disasters) and students endorsing no trauma reported on their mood twice daily for four weeks (Bunce, Larsen, \& Peterson, 1995). While trauma-exposed individuals reported significantly less positive emotion in their daily ratings, their mean negative emotion ratings were not significantly different 
from those of individuals endorsing no trauma. In addition, among trauma-exposed individuals, significant associations were observed between age at the time of trauma exposure and daily positive emotion, such that the earlier in life an individual endorsed experiencing trauma, the less daily positive emotion they reported.

The second study assessed daily emotion and subjective responses to daily stressful events in order to test within-persons associations between daily stress and negative mood among trauma-exposed and nontraumatized men and women (Glaser, van Os, Portegijs, \& Myin-Germeys, 2006). Ten times daily for six days, men and women rated negative mood (i.e., irritated, lonely, anxious, down) and rated how bothered they were by any stressful events that had occurred in the time since their last mood report. Multilevel regression analyses revealed, after accounting for the effects of current depressive symptoms, significant and positive associations between daily stress and daily negative emotion, with stronger associations observed between daily stress and daily negative emotion among those endorsing childhood trauma. Among men and women endorsing childhood trauma, age at childhood trauma also moderated the relationship between stress and negative emotion such that this association was greatest among individuals who reported trauma earlier in childhood (i.e., before age ten).

When these experience sampling studies are considered with the questionnairebased and laboratory studies discussed above, somewhat more consistent patterns begin to emerge. That is, consistent with the questionnaire studies of general emotional experiences and the laboratory findings of Orsillo et al. (2004) and Wagner et al. (2003), these experience sampling studies demonstrate higher mean levels of subjective negative emotion (Muraoka et al., 1998; Beckham et al., 2000; Kashdan, Julian et al., 2006) as 
well as lower mean levels of subjective positive emotion (Beckham et al., 2000; Kashdan, Julian et al., 2006) in the course of everyday life among individuals with PTSD. These findings are also consistent with theories of disrupted emotional processes in PTSD postulating that frequent activation of trauma memory networks among individuals with PTSD results in increased accessibility to negative emotions and decreased accessibility to positive emotion (Litz, 1992; Litz \& Gray, 2002). In addition, taken together, the findings of Glaser et al. (2006) demonstrating greater negative emotional reactions to daily stressful events among traumatized men and women, and those of Kashdan, Uswatte et al. (2006) demonstrating greater emotion instability among male veterans with PTSD, suggest that emotional experiences among individuals with PTSD likely also vary as a result of other factors. Daily diary studies have established that minor stressful daily events are associated with changes in mood among psychologically healthy individuals (Stone, Neale, \& Shiffman, 1993). Likewise, studies of stress reactivity have demonstrated positive associations between daily experiences of stress and daily negative emotional experiences (Marco \& Suls, 1993; van Eck, Nicolson, \& Berkhof, 1998; Mroczek \& Almeida, 2004) and negative associations between daily stress and daily positive emotional experiences (van Eck et al., 1998) among individuals high in trait negative affectivity. Thus, it seems reasonable to conclude that more stable, or "trait" factors such as negative affectivity or neuroticism, and those factors that also vary on a daily basis, such as experiences of stress, also may influence the day-to-day emotional experiences of individuals with PTSD.

In summary, although consistent in several regards with cross-sectional studies of emotional experiences and PTSD, these studies also highlight the importance of 
considering the influence of other daily experiences in examinations of emotional experiences among individuals with PTSD, such as daily stress (e.g., Glaser et al., 2006). In addition, among the four daily emotion studies specifically assessing PTSD, as opposed to trauma exposure, none included women. Thus, given discrepant laboratory findings of relationships between PTSD and emotional responses among men and women, these studies also highlight the need for examining the relationship between PTSD and daily experiences as they occur in the lives of women.

\section{The Present Study}

\section{Overview}

The present study was concerned with associations between PTSD symptom severity and daily emotion among women who have experienced potentially traumatic events. More specifically, it employed experience sampling methodologies to examine discrete emotional experiences, stress, and symptoms of PTSD and used electronic diaries for assessing these daily experiences as they unfolded over the course of one week. It was reviewed and approved by the University of Louisville IRB.

The study sample was focused on women with potentially traumatic experiences of prior violence in intimate relationships (i.e., intimate partner violence; IPV). In general, IPV is defined as physical, sexual, or psychological harm by a current or former spouse or partner (Tjaden \& Thoennes, 2000a). Current definitions of IPV incorporate many physically, sexually, or psychologically aggressive, violent, or otherwise intimidating or threatening behaviors, as well as stalking (e.g., Basile, Arias, Desai, \& Thompson, 2004) and include such acts perpetrated by female partners against male partners (i.e., female-to-male IPV) as well by male partners against females partners (i.e., 
male-to-female IPV) (Tjaden \& Thoennes, 2000b). Because the present study was concerned with emotional experiences among women exposed to potentially traumatic events, IPV in the present study was limited to male-to-female IPV, and IPV exposure was defined as endorsement of physical violence (e.g., being slapped, pushed, punched, kicked), or sexual violence or coercion (e.g., use of force or threats to have sex), or receipt of injury from any such acts by a partner in a prior intimate relationship.

The rationale for selecting women with previous experiences of IPV for the present study sample was as follows. To date, the daily emotional experiences of IPVexposed women have not been studied, despite such women's heightened risk for PTSD development (Golding, 1999) as well as chronic symptoms of PTSD. For example, Woods (2000) demonstrated that nearly $66 \%$ of a sample of community women endorsing prior, but no current, IPV reported that they continued to experience PTSD symptoms at least to a mild degree, despite having been out of their abusive relationships for an average of 9 years. Moreover, $44 \%$ of these women endorsed experiencing moderate to severe reexperiencing symptoms over the week prior to the study, with "reminders bringing back feelings about the abuse" and "experiencing waves of strong feelings“ being endorsed by nearly $50 \%$ of the sample (Woods, 2000, p. 318). Thus, given high rates of chronic PTSD symptoms among women exposed to prior IPV, specifically reexperiencing symptoms, and given theoretical formulations of posttraumatic emotional processes highlighting the importance of reexeperiencing symptoms (e.g., Foa et al., 1989; Horowitz, 1986; Litz \& Gray, 2002), these women seem particularly at risk for long-term disruptions in emotional functioning. 
Finally, for the purposes of eliminating potential confounding effects of age on emotional experiences, the present study sample was limited to IPV-exposed women in midlife. That is, studies of emotion development across the adult lifespan have provided evidence for increasingly complex experiences of emotion as individuals age (e.g., Cartensen, Pasupathi, Mayr, \& Nesselroade, 2000; Ong \& Bergemann, 2004; Charles, 2005), and midlife has been highlighted as a particularly fertile time for these emotional changes to occur (Cartensen \& Charles, 1998).

Aims

In examining daily experiences of emotion, stress, and PTSD symptoms among IPV-exposed women, the present study had four aims. First, given the spirit of "descriptive inquiry" called for in daily process research (Affleck, Zatura, Tennen, \& Armeli, 1999, p. 747), the relative dearth of research examining associations between PTSD and daily emotional experiences in general, and the absence of studies examining daily emotional processes among IPV-exposed women more specifically, the first aim of the present study was descriptive in nature and was motivated by the following question: how are emotional experiences in everyday life among IPV-exposed women characterized? For example, while some have demonstrated that IPV-exposed women report high levels of guilty feelings about their IPV experiences using more global measures of guilt (i.e.. Kubany et al., 1995; Kubany et al.. 1996). are these women's daily emotional experiences also characterized by high levels of guilt, or do feelings of guilt vary from day-to-day? Thus, the first aim of the present study was to provide an ecologically valid characterization of emotional experiences and the contexts in which these emotional experiences occur across the present sample of IPV-exposed women 
through descriptive data on overall levels and variability of specific, discrete emotions experienced within and across days.

Second, as noted, existing laboratory and naturalistic studies have not yet revealed a clear picture of the nature of emotional experiences among individuals with respect to PTSD, and particularly among women. Given this, and given a lack of naturalistic studies examining associations between PTSD and daily emotional experiences among trauma-exposed women, the second aim of the present study was to test associations between PTSD symptom severity and daily emotional experiences among women with prior experiences of IPV.

Third, although consistent positive associations between daily stress and daily experiences of negative emotion have been demonstrated among individuals without trauma exposure, to date, only one study has examined associations between daily stress and daily emotional experiences among trauma-exposed individuals. However, this study did not specifically examine associations between daily stress and emotion with respect to PTSD (i.e., Glaser et al., 2006). Because it seems possible that the "hyperemotionality" observed among individuals with PTSD could be, at least in part, due to greater emotional responses to daily stress, the third aim of the study was to examine the effects of past-month PTSD symptom severity on associations between daily stress and daily emotional experiences among IPV-exposed women.

Finally, although a few self-monitoring treatment studies have assessed PTSD symptoms on a daily basis (Reynolds \& Tarrier, 1996; Tarrier, Sommerfield, Reynolds, \& Pilgrim, 1999; Johnson, Westermeyer, Kattar, \& Thuras, 2002; Simpson et al., 2005), there are no studies to date that have examined relationships between daily emotional 
experiences and the experience of daily PTSD symptoms. Thus, given theoretical explanations closely linking disrupted emotional processes observed in PTSD to the experience of specific PTSD symptoms, the fourth aim of the present study was to investigate temporal relationships between daily emotional experiences and daily PTSD symptoms among IPV-exposed women.

Hypotheses

As stated, given an incomplete understanding of emotional processes and PTSD in general, and given the lack of studies examining emotional processes among women exposed to IPV more specifically, one aim of the present study was to provide descriptive data on specific, or discrete, emotional experiences in everyday life among these women. In addition, in line with the second, third, and fourth aims of the present study, and based on the theoretical and empirical literature reviewed above, the present study also advanced three sets of hypotheses regarding daily emotional experiences, stress, and PTSD symptoms among IPV-exposed women.

A number of qualifications are necessary before outlining specific hypotheses. First, for the purposes of describing emotional experiences as outlined in the first aim of the study, emotions were preserved as "discrete", based on the premise that discrete emotions serve adaptive functions. For example, some have described the experience of sadness as an internal alert to problematic or unpleasant circumstances, while the expression of sadness (e.g., a distressed cry or tears) is believed to function as a social signal that help or comfort is needed (Izard \& Ackerman, 2000). Thus, while sadness experienced frequently and at intense levels may reflect a problem with emotion regulation, experiencing sadness less frequently and at lower levels of intensity might be 
considered adaptive as it may motivate an individual to rectify unpleasant circumstances. In contrast, for the purposes of specifying hypotheses, emotional experiences were classified as either "positive" or "negative". Specifically, following convention in the literature on emotion in general, fear, anger, guilt, shame, sadness, disgust, and contempt were classified in the present study as "negative" emotions, while joy and interest were classified as "positive" emotions. Because surprise has been described as a "neutral" emotion with respect to valence (e.g., Izard, 1977), it was included as neither a negative nor positive emotion, and therefore, was excluded from these classifications. This decision to classify emotions as "positive" and "negative" was based on the fact that there are few available data on discrete emotional experiences and PTSD (i.e., from either laboratory or naturalistic studies) to inform hypotheses with respect to discrete emotions in the present study.

Second, the present study examined the following three different aspects, or facets, of emotional experiences in tests of hypotheses: overall level of emotional experiences, frequency of emotional experiences, and intensity of emotional experiences. Although also described in later sections, for the purposes of outlining hypotheses below, these three facets are also operationalized here. In the present study, overall level refers to a participant"s rating of how much a given emotion was "felt" at the time of assessment, ratings which could range from a score of 1 (not at all) to 5 (very much so) (see Differential Emotions Scale; Materials section). In this way, the overall level of an emotion reflects integrated information about the occurrence of that emotion as well as how intensely it was experienced (e.g., Schimmack \& Diener, 1997). Frequency refers to how often a given emotion was experienced, or how often an emotion occurred. In this 
case, an emotion was said to have occurred if it was rated greater than 1 (e.g., Schimmack \& Diener, 1997), and frequency reflects a count of how many times a given emotion was reported as occurring. Intensity refers to how intensely an emotion was experienced when the emotion actually occurred. In other words, emotion non-occurrence was controlled for by excluding emotion ratings equal to 1 (Schimmack \& Diener, 1997; Zelenski \& Larsen, 2000).

Third, while existing studies of emotional processes and PTSD have classified individuals on the basis of PTSD diagnostic status, the present study retained PTSD as a continuous measure reflecting past-month symptom severity. This decision is based on the general observation that, at any given time in the longitudinal course of chronic PTSD, individuals may not meet full diagnostic criteria but may be experiencing "subclinical" symptoms that are just as distressing or impairing (Blank, 1993). Furthermore, given the findings of Woods (2000) demonstrating the presence of PTSD symptoms several years following the termination of women's abusive relationships, this may be particularly true for the IPV-exposed women in the present study. Hypotheses: Set 1

The first set of hypotheses concern between-persons associations between pastmonth PTSD symptom severity and overall levels, as well as frequency and intensity, of daily positive and negative emotional experiences.

Rationale. As discussed above, the theoretical literature on emotional processes in PTSD posits that the "hyperemotionality" and "hypoemotionality" observed among individuals with PTSD result from multiple negative emotional responses becoming associated with many generalized stimuli in trauma memory networks, rendering 
negative emotional experiences more likely and positive emotional experiences less likely (e.g., Litz, 1992; Litz et al., 2000; Litz \& Gray, 2002). Thus, as more complex and generalized associated negative emotional responses may be present in the trauma memory networks of individuals with more severe PTSD symptoms, it is reasoned that IPV-exposed women with more severe past-month PTSD symptoms should evidence greater frequency and greater intensity of daily negative emotions and should evidence less frequent and less intense daily positive emotion. In addition, other individual difference factors may also be expected to affect relationships between daily emotional experiences and PTSD. That is, given individual differences in the tendency to experience more frequent negative emotion in general (i.e., trait affectivity), it follows that this likely influences daily emotional experiences and should also be controlled for when examining associations between PTSD and frequency and intensity of daily emotions. Thus, the following was hypothesized:

Hypothesis la. After accounting for the effects of trait negative affectivity, women with greater past-month PTSD symptom severity will evidence higher overall levels, higher frequency, and higher intensity of daily negative emotions.

Hypothesis $1 \mathrm{~h}$. After accounting for trait negative affectivity, women with greater past-month PTSD symptom severity will evidence lower overall levels, lower frequency, and lower intensity of daily positive emotions.

Hypotheses: Set 2

These hypotheses concern between-persons differences with respect to pastmonth PTSD symptom severity on within-persons associations between daily stress and daily emotions. 
Rationale. As previously described, studies of emotional reactivity to stress have revealed positive associations between daily experiences of stress or stressful events and the experience of negative emotion (e.g., Marco \& Suls,1993; van Eck et al., 1998; Mroczek and Almeida, 2004) and negative associations between daily stress and positive emotion (e.g., van Eck et al., 1998) among men and women, including those with prior trauma exposure (e.g., Glaser et al., 2006). Likewise, in the two laboratory studies of subjective emotional responses to non-traumatic emotional stimuli conducted among women specifically, women with PTSD responded with increased negative emotional responses to both negative and positive stimuli (Orsillo et al., 2004) and increased selfreported emotional arousal to both negative and positive stimuli regardless of whether trauma priming had occurred (Wagner et al., 2003).

Taken together, these findings suggest that, while the increased negative emotion observed in PTSD may directly result from more frequent activation of trauma memory networks as some have theorized (Foa et al., 1989; Litz, 1992), the increased negative emotion in PTSD may also reflect increased negative emotion to daily experiences overall, and more specifically, daily encounters with stress (i.e., Glaser et al., 2006). As such, it is reasoned that women with greater severity of PTSD symptoms will evidence greater negative emotion and less positive emotion on days that they perceive as stressful. However, given evidence suggesting that individuals high in trait negative affectivity (e.g., Marco \& Suls, 1993; Mroczek \& Almeida, 2004) react with greater negative in emotion in response to daily stressful events in general, trait negative affectivity likely also influences within-person relationships between daily emotional experiences and 
stress among individuals with PTSD and should be included as a between-persons covariate in such examinations. Thus, the following was hypothesized:

Hypothesis 2a. Although a positive within-person association between daily stress and daily negative emotion is expected for women overall, it is expected that, when accounting for the effects of trait negative affectivity, the within-person association between daily stress and daily negative emotion will be greater among women with more severe past-month PTSD symptoms.

Hypothesis $2 b$. Likewise, although a negative within-person association between daily stress and daily positive emotion is expected for women overall, it is expected that, when accounting for the effects trait negative affectivity, the within-person association between daily stress and positive emotion will be greater among women with more severe past-month PTSD symptoms.

\section{Hypotheses: Set 3}

These hypotheses test theoretical propositions of disrupted emotional processes in PTSD via examination of within-day associations between emotional experiences and specific symptoms clusters as well as temporal relationships between daily emotional experiences and specific PTSD symptom clusters.

Rationale. As previously mentioned, no published studies to date have examined temporal relationships between emotional experiences and PTSD symptoms. Theoretical formulations of emotional disruptions in PTSD link the intense experiences of negative emotion observed among individuals with PTSD to reexperiencing the trauma (Foa et al., 1998; Horowitz, 1986; Litz, 1992) and describe emotional numbing as a defense against the painful and intense emotion arising from continually reexperiencing the traumatic 
event (Horowitz, 1986). Thus, it is reasoned that individuals who have higher levels of past-month reexperiencing symptoms likely also reexperience traumatic memories more frequently in daily life and also experience higher levels of daily negative emotion as a result of this reexperiencing. Furthermore, in line with theories postulating cycles of reexperiencing and avoidance/numbing (Foa at al., 1995; Horowitz, 1986), it is reasoned that women who experience more severe levels of reexperiencing on a given day will also experience more severe emotional numbing symptoms in the days to follow. However, this relationship is expected to be moderated by daily negative emotion. That is, severe reexperiencing symptoms on a given day are expected to interact with same-day negative emotion to predict severity of numbing symptoms on subsequent days.

Hypothesis $3 a$. Severity of daily reexperiencing symptoms will be positively associated with intensity of daily negative emotion. This within-person relationship is expected to be strongest among women with more severe past-month reexperiencing symptoms.

Hypothesis $3 h$. Severity of reexperiencing symptoms on a given day will be positively associated with emotional numbing symptoms on subsequent days. This within-persons lagged relationship between reexperiencing symptoms and numbing symptoms will be moderated by negative emotional responses, such that greater severity of numbing symptoms will be observed following days with more severe reexperiencing and more intense negative emotional experiences.

\section{Statistical Power and Sample Size Calculations}

Power analyses were first conducted to inform recruitment strategies and to determine necessary sample sizes for hypothesis testing. For tests of main effects of 
PTSD on mean frequency and intensity of daily emotion as outlined in hypotheses la and 1b, power analyses were performed using GPOWER software (Faul \& Erdfelder, 1992). Results were based on a significance level of .05 and two predictors per model (i.e., one control and one explanatory variable). Using the data presented by Beckham et al. (2000) and Muraoka et al. (1998), calculated effect sizes of PTSD on mean levels of daily negative emotion were considered large by conventional classifications (i.e., Cohen ${ }^{*} d$ >.8; Cohen, 1988). Using the data presented by Beckham et al. (2000) and Bunce et al. (1995), calculated effect sizes of PTSD on mean daily positive emotion were also considered large (Cohen, 1988). Likewise, Kashdan, Julian et al. (2006) reported a large effect size of PTSD on intensity of daily negative emotion (i.e., $r=.55$ ) and a medium effect size of PTSD on daily positive emotion (i.e., $r=.32$ ) by conventional classifications (Cohen, 1988). Thus, a medium-large effect size $\left(f^{2}=.25\right)$ was used in power analyses and sample size calculations for the purposes of testing main effects of PTSD on daily negative and positive emotion frequency and intensity as outlined in hypotheses $1 \mathrm{a}$ and $1 \mathrm{~b}$. Analyses indicated that given two predictors, a sample size of 42 was needed to achieve power of .80 for detection of a medium-large effect using ordinary least-squares (OLS) regression.

A power analysis was also conducted for estimating optimal sample size for multilevel models tested in hypotheses $1 \mathrm{a}-\mathrm{b}$ and $2 \mathrm{a}-\mathrm{b}$ using the Optimal Design for Multilevel and Longitudinal Research software, version 1.77 (ODS; Liu, Spybrook, Congdon, Martinez, \& Raudenbush, 2006). For multilevel regression analyses, power is represented as a function of the number of individuals $(J)$; number of within-persons observations, or clusters $(n)$; the intraclass correlation coefficient, representing between- 
cluster variability $(\rho)$; and the expected effect size (d) (Spybrook, Raudenbush, Liu, \& Congdon, 2006). From the one study examining associations between daily stress and daily negative emotion among trauma-exposed individuals (i.e., Glaser et al., 2006), $\rho$ was calculated to be approximately.1. However, given no existing data from which to estimate an expected effect size of PTSD on associations between daily stress and daily emotion or temporal associations between daily emotion and daily PTSD symptoms, a series of three analyses were conducted to determine optimal sample size $(J)$ given small, medium, and large effect sizes, respectively, at a significance level $=.05, \rho=.1$, and $n=$ 35 (i.e., although the sampling procedure resulted in a maximum of 49 within-persons observations, this estimate of $n$ accounts for expected missing data among the majority of participants). Analyses indicated that to achieve power $=.80$, sample sizes of 65,27 , and, 12 are needed for detection of small, medium, and large effects, respectively, based on conventional classifications (Cohen, 1988). Thus, considering the sample size calculations for hypotheses $1 \mathrm{a}-\mathrm{b}$ and $2 \mathrm{a}-\mathrm{b}$ along with feasibility and cost constraints, $\mathrm{a}$ sample size of 30 was determined as adequate for hypotheses testing in the present study. However, because it was expected that some cases would be excluded from the final sample as a result of missing diary data, a decision was made to oversample to account for this. Thus, a sample size of 40 was targeted in order to achieve a final sample size of 30. 


\section{METHODS}

\section{Participants}

Fifty-three women endorsing prior IPV (i.e., IPV from a former intimate partner at any time during the relationship) but no current IPV (i.e., no IPV from either a former or current intimate partner in the past year) were contacted after participating in a larger study on stress and aging among women. In response to newspaper advertisements, cards mailed to women's homes, and flyers posted in the community targeting women divorced or separated from a past "stressful relationship", women called the study office and completed a phone interview to determine if the following eligibility criteria were met for participation in the larger study: comfortable writing and reading English; no hospitalization for a psychiatric or emotional condition in the past six months; divorced or separated for at least one year from a partner to whom the woman was married or with whom she was cohabitating; and no endorsement of current IPV as assessed by the STaT (Paranjape \& Liebschutz, 2003; see below for a description of this measure).

Women were then recruited into the present study in the following manner. First, women's research records from the larger study were reviewed to establish that, in addition to fulfilling the above eligibility criteria of the larger study, eligibility criteria for participation in the present study were also met. Specifically, criteria for the present study included the following: history of IPV in a prior relationship; no current alcohol abuse/dependency or use of street drugs; no current employment other than first shift 
(i.e., roughly between the hours of $8 \mathrm{am}$ and $5 \mathrm{pm}$ ); and, because the present study focuses specifically on women in midlife, between 45 and 60 years of age (e.g., Cartensen et al., 2000). In the present study, history of IPV was defined as experiencing at least one act of physical violence (e.g., being punched or hit with something that could hurt) or sexual coercion (e.g., being threatened or forced to have sex), or being injured by a partner (e.g., feeling physical pain the next day because of a fight) in a prior intimate relationship. This was assessed using the Revised Conflict Tactics Scale (CTS2; Straus, Hamby, Boney-McCoy, \& Sugarman, 1996; see below for a description of this measure). Women meeting eligibility criteria for the present study and who, during participation in the larger study had given permission to be re-contacted about future studies through our laboratory, were either contacted by phone or sent a letter informing them that were eligible for a new study on women's daily stress and emotions and were invited to participate. Interested women then called the study office to schedule a session and to enroll in the present study. Of the 53 women contacted and invited to participate, 39 women enrolled in the present study. Thirty-seven women completed the entire sevenday protocol, while one woman discontinued the study after five days of participation, citing increased time constraints in her schedule. One participant's diary data were not available due to accidental vehicular destruction of the electronic diary (i.e., personal digital assistant) during her participation.

\section{Materials}

Personal Digital Assistants

All daily experience assessments were completed electronically using a Palm Pilot Z22 handheld personal digital assistant (PDA). Questionnaires to assess daily 
experiences were programmed onto the PDAs via the Experience Sampling Program v. 4.0, an open-source software program made available for public use (ESP; Barrett \& Feldman Barrett, 2000). In contrast to paper-and-pencil diaries, the use of electronic diaries eliminates the need for additional signaling devices, such as wristwatches or pagers, as the PDA acts as both the recording device and signaling device, and ensures participant adherence to sampling procedures in that electronic assessments are date- and time-stamped (Bolger et al., 2003). The size of these devices also contributes to ease of portability as they are small enough for carrying in a purse or pocket (i.e., dimensions of the Palm Pilot Z22 used in the present study are $10 \mathrm{~cm} \times 6.5 \mathrm{~cm} \times 1.5 \mathrm{~cm}$ with a color screen measuring $4.5 \mathrm{~cm} \times 4.5 \mathrm{~cm}$ ). Participants in studies using handheld electronic PDAs have provided favorable reports with respect to their use (Feldman Barrett \& Barrett, 2001) and have reported that they find them less stigmatizing for symptom recording compared to paper-and-pencil diaries (Schärer et al., 2002). Additionally, previous studies have successfully employed similar handheld electronic recording devices among samples of varying ages, levels of socioeconomic status, and levels of psychological functioning (Zeman, Johnson, Arfkin, Smith, \& Opoku, 2006; Burton, Weller, \& Sharpe, 2007).

\section{Measures of Between-Persons Factors}

Measures of self-reported sociodemographic variables (e.g., age, ethnicity, annual household income, highest level of education attained, occupational status, marital status) and the following measures of relevant between-persons factors were either administered during women's scheduled visits to the laboratory for their participation in the larger study or during their scheduled visits for participation in the present study. The one 
exception is the STaT, which was administered during the phone interview as previously noted. Measures administered during women's participation in the larger study were completed on a Dell Latitude 820 laptop computer using Snap Interviewer software v. 9.02 for offline electronic survey administration (Snap Surveys Ltd., 2006). All other measures for the present study (with the exception of daily experience measures) were administered in paper-and-pencil format.

Screening Measures

STaT. The three-item STaT was designed as a screening instrument for lifetime IPV exposure among women (Paranjape \& Liebschutz, 2003). One study demonstrated a sensitivity of $96 \%$ and specificity of $75 \%$ among a sample of women recruited from an emergency department when IPV when compared with interview-determined lifetime IPV estimates (Paranjape \& Liebschutz, 2003). As previously mentioned, the STaT was administered during the phone interview of the larger study for the purposes of identifying and excluding callers with current experiences of IPV and was modified to reflect IPV experiences in the past year. Women were excluded if they endorsed any one of the three STaT items (i.e., in the past year, has an intimate partner "pushed or slapped you", "threatened you with violence", or "thrown, broken, or punched things in your presence"?).

The Revised Conflict Tactics Scales. The Revised Conflict Tactics Scales (CTS2; Straus, Hamby, Boney-McCoy, \& Sugarman, 1996) assesses frequency of violent or abusive acts committed by a partner in the context of an intimate relationship (i.e., psychological aggression, physical violence, sexual coercion, and injury). The CTS2 consists of 39 items such as "My partner punched or hit me with something that could 
hurt" that are rated on a scale from 0 (never) to 6 (more than 20 times) in an intimate relationship over the past year. Cronbach`s alphas ranged from.79 to .95 for the subscales comprising the CTS2 (Straus et al., 1996). As previously mentioned, the CTS2 was used for the purposes of identifying women with histories of IPV for inclusion in the present study. To accomplish this, the instruction set was changed to include at any time during an intimate relationship, and women who endorsed ever experiencing an act of physical violence, sexual coercion, or being injured were included for the present study. PTSD Checklist - Civilian Version

The PTSD Checklist - Civilian Version (PCL-C; Weathers, Litz, Herman, Huska, \& Keane, 1993) was used to assess past-month PTSD symptom severity. The 17 items of the PCL-C correspond to the 17 PTSD symptoms as outlined in the DSM-IV, and the instructional set and items of the PCL-C may be worded so that symptom reporting is anchored to a specific event or may be worded so that symptoms are reported more broadly in reference to "stressful experiences in the past" (Norris \& Hamblen, 2004). Because the present study sought to investigate associations between daily experiences and PTSD symptoms more generally, the latter instructional set was used. Individuals rate how much they have been "bothered" by symptoms such as "repeated, disturbing memories, thoughts, or images of a stressful experience from the past" over the past month using a scale of 1 (not at all) to 5 (extremely), yielding a possible total symptom severity score ranging from 17-85. Severity scores for individual symptom clusters may also be generated. In general, versions of the PCL have demonstrated excellent psychometric properties and correlate highly with structured interview assessments of PTSD, such as the Clinician-Administered PTSD Scale (CAPS; Norris \& Hamblen, 
2004). Among a sample of women exposed to IPV, Cronbach's alpha for the PCL was .93 (Krause, Kaltman, Goodman, \& Dutton, 2006). Cronbach`s alpha for the present sample was .89 .

The Big Five Inventory

The Big Five Inventory (BFI; John, Donahue, \& Kentle, 1991) is a 44-item personality measure which yields scales corresponding to each of the traits within five factor models of personality (i.e., extraversion, agreeableness, conscientiousness, neuroticism, and openness). Items such as "I see myself as someone who can be tense" and "I see myself as someone who has a forgiving nature" are rated on a scale ranging from 1 (disagree strongly) to 5 (agree strongly), and scale scores are calculated as the mean score of items from their respective trait scales. The BFI has demonstrated adequate psychometric properties, including internal consistency and test-retest reliability, and self- and other-report versions of the BFI demonstrate high levels of agreement (e.g., Srivasta, John, Gosling, \& Potter, 2003). For the present study, scores from the eight-item neuroticism scale were used as a measure of trait negative affectivity, yielding possible scaled scores ranging from one to five. In the present sample, the neuroticism scale demonstrated adequate internal consistency (Cronbach’s alpha $=.78$ ).

\section{Measures of Within-Persons Factors: Daily Experiences}

\section{Daily Emotional Experiences}

Evidence suggests that while emotional traits are best captured using dimensional measures, momentary emotional experiences are best represented as discrete (Zelenski \& Larsen, 2000; Vansteedlandt, Van Mecchelen, \& Nezlak, 2005). Thus, the Differential Emotions Scale-IV (DES-IV; Izard, Libero, Putnam, \& Haynes, 1993) was selected for 
assessment of daily emotional experiences. The 36-item DES-IV consists of ten threeitem scales corresponding to each of the ten fundamental discrete emotions set forth by differential emotions theory (i.e., fear, anger, guilt, shame, disgust, contempt, joy, interest, and surprise; Izard, 1977) in addition to two three-item scales measuring shyness and self-hostility. Designed to assess individuals' typical emotional experiences in daily life, items such as "Feel like things are so rotten they could make you sick" (i.e., disgust) and "Feel like screaming at someone or banging on something" (i.e., anger) are rated on a scale from 1 (rarely or never) to 5 (very often) using the instructional prompt, "In your daily life, how often do you...?" For use in the present study, this instructional prompt was adapted in order to assess momentary emotional experiences (i.e., "Right now, how much do you...?") (e.g., Cerrin \& Barnett, 2006). Correspondingly, the rating scale of the DES-IV was adapted in order to reflect ratings of intensity of momentary emotional experiences, ranging from 1 (not at all) to 5 (very much so). In this way, in addition to the intensity of a given emotion, data on the presence (i.e., a rating greater than 1) or absence (i.e., a rating equal to 1) of the emotion was also provided (e.g., Shimmack \& Diener, 1997). Studies utilizing daily diary designs have successfully employed the DES-IV for the assessment of daily emotional experiences (e.g., Cerrin \& Barnett, 2006). The 12 scales of the DES-IV were determined empirically through factor analytic methods, and a recent review of 30 studies reported adequate reliability across samples varied in age, SES, and gender for each of the 12 scales (mean Cronbach's alphas ranged from .56 to $.75 ;$ Youngstrom \& Green, 2003). However, given that the present study design required participants to complete multiple daily assessments of emotional experiences, a shortened version for use in the present study was desired in the interest of 
minimizing participant burden. Thus, the ten items demonstrating the highest factor loading on each of their respective emotion scales from the factor analytic data presented by Izard et al. (1993) were selected for use in the present study on the premise that these ten items were the most representative of each of the ten fundamental emotions. This strategy has been used to create an unpublished "short form" of the DES-IV, which has demonstrated adequate psychometric properties in adult samples (C.E. Izard, personal communication, July 3, 2007). However, it was noted that there was a disproportionate number of items assessing emotions typically classified as "negative" (i.e., sadness, anger, fear, guilt, shame, contempt, disgust) as compared to those assessing emotions typically classified as "positive" emotions (i.e., interest, joy). Thus, the DES-IV short form was further modified for use in the present study by adding the remaining two items assessing joy and the two remaining items assessing interest from the original DES-IV. This modification resulted in a total of 14 items which captured the experience of 10 discrete emotions for the daily experience assessment measure in the present study (i.e., 7 "negative" emotion items, 6 "positive" emotion items, and 1 "neutral" emotion item).

\section{Daily Emotional Assessment Context}

The social context in which emotional experiences were rated at each signal was captured with the question: "Which best describes your surroundings in the time leading up to this last signal?" Women selected responses from the following forced-choice responses: alone; with pets; with family; with friends; with significant other, spouse; with ex-partner, ex-spouse; with co-workers; or with strangers, acquaintances. Likewise, the following question assessed the nature of activities in the immediate context surrounding each signal: "Which best describes your activity in the time leading up to this last 
signal?" Women selected responses from the following forced-choice responses: leisure activity or hobby; resting; working at job; running errands, doing chores; socializing; or other.

Daily Stress

Measurement of daily stress was accomplished using the following item from the work of Brantley, Waggoner, Jones, \& Rappaport (1987): “Overall, how stressful was today for you?" Responses reflect global ratings of daily stress rated on a scale from 0 (no stress) to 10 (the most stressful day I have had). Global daily stress ratings as determined by the above item were found to significantly and positively correlate with number and severity of stressors reported each day for each of 28 consecutive days via the Daily Stress Inventory (Brantley et al., 1987), suggesting that daily levels of stress may be reliably captured using more global, single-item daily assessments.

\section{Daily PTSD Symptoms}

In addition to measuring severity of past-month PTSD symptoms, the PCL-C (Weathers et al., 1993) was also used to assess the severity of daily PTSD symptoms. Likewise, because the present study was concerned with daily PTSD symptoms more generally, the instructional set was worded so that symptoms are reported in reference to "stressful experiences in the past" rather than in reference to a specific event (Norris \& Hamblen, 2004). Also, for the purposes of assessing daily symptoms, the instructional set was adapted from the time reference of "past month" to "today".

\section{Procedure}

\section{Study enrollment and PDA training}


Each participant attended an initial one-hour, one-on-one laboratory session with the experimenter. Study procedures were first explained in detail by the experimenter, and following provision of informed consent for their participation, women were enrolled in the study and provided with a study ID number. After completing paper-and-pencil measures, women were trained on the sampling protocol and use of the PDA. It is recognized that, by their nature, experience sampling designs are time-intensive and could be perceived as intrusive by participants (Stone \& Litcher-Kelly, 2006). Although some have noted that extensive training may be needed to ensure participant adherence to experience sampling protocols, others have suggested that training be as brief as possible (Palmblad \& Tiplady, 2004) and have placed more emphasis on participant understanding of study aims and communicating the value of participants' involvement, as this has been demonstrated to increase participant motivation and adherence to experience sampling protocols (Christensen, Feldman Barrett, Bliss-Moreau, Lebo, \& Kaschub, 2003). Thus, for the present study, PDA training time was approximately 30-40 minutes for each participant and served to achieve the following goals: to establish rapport with the participant; to facilitate understanding of the purpose of the study and the value of their participation; and to acquaint the participant with the PDA and its use via experimenter verbal instruction and demonstration.

During the standardized training session, women were instructed on sampling procedures (e.g., timing of PDA signals), became acquainted with the features of the PDA (e.g., how to use the stylus), and completed a practice questionnaire on food preferences using the PDA. Women also completed one practice emotion assessment in the presence of the experimenter so that it could be ascertained that the participant 
understood the procedure for completing assessments and how to indicate responses using the PDA when signaled. Any questions about the sampling procedure, the equipment, or the assessment instruments employed were clarified by the experimenter at this time. Women were also asked about their typical schedules (i.e., when they normally rise in the morning and when they typically go to bed) to ensure that PDA signals for daily assessments fell within the time frame of each participant's typical day. Written instructions and help tips (i.e., a "cheat sheet") for operating the PDA and problemsolving potential difficulties were provided to participants to take with them for reference. Although the experimenter's contact phone numbers were provided and women were encouraged to call at any time should any problems arise, a phone "checkin" was also scheduled for the day following each woman`s first day of study participation. In this way, the experimenter was alerted to any problems or equipment malfunctions and could clarify any questions that may have arisen during initial sampling (Christensen et al., 2003).

Women were compensated $\$ 50.00$ for their participation, which they received following the training period at the close of the initial laboratory session, and women were informed that the compensation was theirs to keep even if they chose to discontinue the study before the seven day sampling period was completed. This decision was based on literature suggesting that providing compensation in advance of participation, particularly to individuals participating in longitudinal research and those which involve a significant time commitment, such as the present study, "involves [participants] in a research relationship" and promotes a "trusting relationship" between a participant and 
researcher (Collins, Ellickson, Hays, \& McCaffery, 2000, p. 349), which may increase adherence to such study protocols.

\section{Experience Sampling Procedures}

The sampling design of the present study was similar to existing experience sampling studies of daily emotional experiences among community samples (e.g., Cerin \& Barnett, 2006), including samples employing midlife women (e.g., Cartensen et al., 2000). For consistency among participants, the day following the visit to the laboratory and receipt of the PDA marked "day 1" and the start of the study for each woman. Sampling spanned one week, or seven consecutive days. For the present study, the unit of "day" is defined as 9:00 a.m. to 9:00 p.m. and is divided into seven equal blocks of 1 h 42 min each (i.e., 9:00 a.m. to 9:00 p.m. $=720$ minutes $/ 7$ blocks $=1 \mathrm{~h} 42 \mathrm{~min}$ each block). Women were signaled by the PDA randomly once during each of the following seven blocks throughout the day: block $1=9: 00$ a.m. to $10: 42$ a.m.; block $2=10: 42$ a.m. to $12: 24$ p.m.; block 3: 12:24 p.m. to 2:06 p.m.; block 4: 2:06 p.m. to 3:48 p.m.; block 5: 3:48 p.m. to 5:30 p.m.; block 6: 5:30 p.m. to 7:12 p.m.; and block 7: 7:12 p.m. to 9:00 pm. Random signals were chosen over fixed-interval signals in order to decrease predictability of signals by participants, which may influence reporting of momentary emotional experiences (Stone \& Litcher-Kelly, 2006; Bolger et al., 2003). The unit of "day" was adjusted for two women who noted that they did not typically rise before 9:00 a.m. For these women, the unit of day was defined as 10:00 a.m. to 10:00 p.m.; however, signaling procedures remained otherwise unchanged (i.e., followed the same $1 \mathrm{hr} 42 \mathrm{~min}$ block design as outlined above). 
Henceforth, each set of diary ratings at a signal is referred to as an "assessment". When signaled by the PDA, women first answered two forced-choice questions assessing social and activity context leading up to the signal and then reported on their momentary emotional experiences. Women had five minutes to respond to the PDA upon being signaled initially; however, in the event that no response was initiated within five minutes, the PDA re-signaled after five minutes. Five minutes following the second signal, data for that assessment was recorded by ESP as "missing" if no response was initiated at that time. A feature of ESP intended to reduce the amount of missing data in experience sampling studies, this re-signaling practice allows some flexibility in responding for women should the signal occur at an inconvenient time (e.g., while driving) without compromising momentary assessment of emotional experiences (Feldman Barrett \& Barrett, 2001).

Daily stress and daily PTSD symptoms were measured once daily using an endof-day diary format. In this way, ratings of daily stress and PTSD symptoms reflect those occurring over the course of the day rather than actual momentary experiences. Specifically, the PDA was programmed such that women completed measures of daily PTSD symptoms and daily stress immediately following their seventh and final emotion assessment of each day. Paper questionnaires of daily PTSD symptoms and daily stress were provided to each participant to complete as "backup" in the event that they missed the final signal of the day (and thus were not able to complete measures of daily PTSD symptoms and daily stress using the PDA).

The final PTSD symptom rating on day seven marked the end of the sampling period for the study, at which time the PDA alerted participants that the study was 
complete. Women then either contacted the study office or were contacted by the experimenter to schedule a convenient time for returning the PDA to the laboratory. During their second visit to the laboratory, women completed a debriefing interview regarding their experiences as participants and any negative reactions they had to completing the study protocol, and referrals for treatment services were available if needed. Participants who found a second trip to the laboratory inconvenient or not possible were given the option of having the PDA retrieved by the experimenter at either the participant"s home or a designated public meeting place of the participant's choice (e.g., library, restaurant, shopping center).

\section{Data Analysis}

The above sampling procedures produced "nested" data. or data that are arranged in "person-days", with daily assessments of emotion. stress, and PTSD symptoms nested within women across days. Specifically, with respect to emotion assessments, the above sampling procedures resulted in a maximum of 49 within-day emotion assessments per woman (i.e., 7 days $x 7$ blocks per day), with each within-day emotion assessment comprised of fourteen continuous variables representing level of momentary emotional experience for ten specific emotions as described in the Materials section above (i.e., one variable each for the emotions of fear, anger, guilt, shame, sadness, disgust, contempt and surprise, and three variables each for the emotions of joy and interest). With respect to daily stress and daily PTSD symptoms, the above sampling procedures resulted in a maximum of seven within-persons assessments of daily stress and maximum of seven within-persons assessments of daily PTSD symptoms.

\section{Data Reduction Strategies}


Overall level of emotion. For testing associations between past-month PTSD symptom severity and overall levels of negative and positive emotion (as outlined in hypotheses $1 \mathrm{a}$ and $1 \mathrm{~b}$ ), and for testing the moderating effect of past-month PTSD symptom severity on within-persons associations between daily stress and daily negative and positive emotion (as outlined in hypotheses $2 \mathrm{a}$ and $2 \mathrm{~b}$ ), emotional experiences were preserved as reported (i.e., without separating frequency and intensity components) and thus reflect overall levels of positive and negative emotion experiences, taking into account both frequency and intensity of emotions (Schimmack \& Diener, 1997). To calculate negative emotion level and positive emotion level variables, within-signal means were created by averaging ratings across all negative emotion items and ratings across all positive emotion items, respectively, within each signal. Thus, for these analyses, the temporal organizing unit is at the level of signal.

Because hypotheses $3 \mathrm{a}$ and $3 \mathrm{~b}$ concerned time-dependent associations across days, negative emotion level variables were calculated within days rather than within signals. This was accomplished by averaging all momentary negative emotion ratings across signals within a given day. This strategy reduced the resulting data for testing hypotheses $3 a-b$ to one average negative emotion level score per day (i.e., seven negative emotion level scores) for each woman. Thus, for these analyses, the temporal organizing unit is at the level of day.

Emotion frequency. For testing associations between past-month PTSD symptom severity and negative emotion frequency as specified in hypothesis la, counts, or frequencies, were tabulated for each negative emotion (i.e., fear, anger, sadness, guilt, shame, disgust, contempt) across the week and then summed across negative emotions. 
Counts were generated on the basis of the occurrence of a negative emotion, with an occurrence defined as any negative emotion rating greater than 1 (Schimmack \& Diener, 1997). Because the number of completed assessments differed between women (i.e., some women had fewer assessments than others due to missing a signal), negative emotion frequency was represented as a proportion of a woman's count of negative emotions to the total possible counts of negative emotions for that woman. The same strategy was used to calculate frequency of positive emotion using joy and interest ratings for testing associations between past-month PTSD severity and positive emotion frequency as outlined in hypothesis $1 \mathrm{~b}$. Thus, the temporal organizing unit for these analyses is at the level of week.

Emotion intensity. For testing associations between past-month PTSD severity and negative emotion and positive emotion intensity (as specified in hypotheses $1 \mathrm{a}$ and 1b), intensity scores were calculated for only positive and negative emotions that occurred (i.e., received ratings greater than 1). Intensity scores were then averaged across days and across all negative emotion items and positive emotion items to create mean negative emotion and mean positive emotion intensity scores, respectively. Thus, for these analyses the temporal organizing unit for these analyses is also at the level of week.

\section{Tests of Hypotheses}

For testing hypotheses regarding relationships between past-month PTSD symptom severity and daily emotion frequency and intensity as outlined in hypotheses 1a and $1 \mathrm{~b}$ (in which the temporal organizing unit was week), ordinary least-squares (OLS) 
hierarchical regression models were used. Models were tested using SAS PROC REG (v. 9.2 SAS Institute).

Multilevel modeling techniques for nested, or hierarchical, data were selected for testing associations between past-month PTSD symptom severity and daily emotion levels as outlined in hypotheses $1 \mathrm{a}$ and $1 \mathrm{~b}$ (in which the temporal organizing unit was signal); for testing associations between past-month PTSD, daily stress, and daily emotion as outlined in hypotheses $2 \mathrm{a}$ and $2 \mathrm{~b}$ (in which the temporal organizing unit was signal); and for testing temporal associations between daily emotion and daily PTSD symptoms as outlined in hypotheses $3 \mathrm{a}$ and $3 \mathrm{~b}$ (in which the temporal organizing unit was day) (Raudenbush \& Bryk, 2002). In these approaches, a within-person (i.e., level1) regression equation is first modeled for estimation of each individual's unique intercept and slope. Within-person intercepts and slopes can then be then modeled as outcomes to be explained by between-persons (i.e., level-2) factors, and a full model combining level-1 and level-2 equations is generated for the simultaneous examination of within- and between-persons factors (Raudenbush \& Bryk, 2002).

Multilevel modeling approaches have been recommended specifically for the analysis of data generated from experience sampling methods (Schwartz \& Stone, 1998; Affleck et al., 1999; Bolger et al., 2003), and offer several advantages over traditional statistical approaches for repeated measures designs. First, multilevel modeling approaches circumvent the problem of non-independence among observations inherent in repeated, within-persons designs by incorporating a random effect into the model which is unique to each person (Raudenbush \& Bryk, 2002; Schwartz \& Stone, 1998). Second, they allow for inclusion of persons with missing data (Nezlak, 2001). Third, unlike other 
approaches used for nested data, multilevel modeling approaches do not require equal spacing of assessments within persons (Schwartz \& Stone, 1998) or consistency in the times of assessment across persons (King, King, Salgado, \& Shalev, 2003). In the present study, multilevel models were tested using HLM v. 6.04, student version (Raudenbush, Bryk, \& Congdon, 2007). All models were run using restricted maximum likelihood estimation (Schwartz \& Stone, 1998; Raudenbush \& Bryk, 2002). Hypotheses: Set 1

For testing hypotheses regarding relationships between past-month PTSD symptom severity and daily emotion frequency and intensity, a series of four OLS hierarchical regression models were used. For each dependent measure (i.e., daily negative emotion frequency, daily negative emotion intensity, daily positive emotion frequency, and daily positive emotion intensity), a hierarchical regression model was constructed with trait negative affectivity entered as a control variable on step 1 and pastmonth PTSD symptom severity on step 2. For these analyses, predictors (i.e., trait negative affectivity and PTSD symptom severity), were centered around their respective grand means (Aiken \& West, 1991).

For testing associations between past-month PTSD symptom severity and daily negative and positive emotion levels, two multilevel regression models were used. Level of negative and level of positive emotion served as dependent measures in the first and second models, respectively, while trait negative affectivity and past-month PTSD symptom severity served as level-2 predictors and were centered around their respective grand means. In this way, the intercept reflects the predicted level of negative or positive emotion at a given signal for an individual whose trait negative affectivity and PTSD 
scores are equal to the sample mean (Raudenbush \& Bryk, 2002; Nezlek, 2001; Nezlak, $2003)$.

The level-1 equation (1), level-2 intercept equation (2), and the combined full model (3) for testing associations between PTSD symptom severity and daily emotion are specified below. In these equations, EMOTION represents level of daily negative or positive emotion, NA represents trait negative affectivity, and PTSD represents pastmonth PTSD symptom severity.

$$
\operatorname{EMOTION}_{\mathrm{ij}}=\beta_{0 \mathrm{j}}+\mathrm{r}_{\mathrm{ij}}
$$

Intercept: $\beta_{0 \mathrm{j}}=\gamma_{00}+\gamma_{01}\left(\mathrm{NA}_{\mathrm{j}}\right)+\gamma_{02}\left(\mathrm{PTSD}_{\mathrm{j}}\right)+\mathrm{u}_{0 \mathrm{j}}$

$$
\text { EMOTION }_{\mathrm{ij}}=\gamma_{00}+\gamma_{01}\left(\mathrm{NA}_{\mathrm{j}}\right)+\gamma_{02}\left(\mathrm{PTSD}_{\mathrm{j}}\right)+\mathrm{u}_{0 \mathrm{j}}+\mathrm{r}_{\mathrm{ij}}
$$

In the level-1 equation (1), the predicted level of daily emotion at signal $i$ for woman $j$ is modeled as a function of woman $j$ s mean level of daily emotion $\left(\beta_{0 \mathrm{j}}\right)$ and a unique residual term for woman $j$ at signal $i$. In the level-2 intercept model (2), woman $j$ 's intercept is modeled as a function of the intercept for all women, or mean emotion level for the entire sample, $\left(\gamma_{00}\right)$, the effects of trait negative affectivity $\left(\gamma_{01}\right)$ and past-month PTSD symptom severity $\left(\gamma_{02}\right)$, and the unique effect of woman $j$ on mean daily emotion. Substituting equation (2) into equation (1) generates the full model, equation (3), in which daily emotion for woman $j$ for signal $i$ is modeled as a function of the overall sample mean $\left(\gamma_{00}\right)$, main effects of between-persons factors (i.e., trait negative affectivity 
and past-month PTSD symptom severity), and both within- and between-persons residual terms.

Hypotheses: Set 2

Two multilevel regression models were also used for testing associations between PTSD, daily stress, and daily negative and positive emotion. As in hypotheses $1 \mathrm{a}$ and $1 \mathrm{~b}$, daily negative emotion level and positive emotion level served as dependent measures in the first and second models, respectively. In both models, daily stress served as the level1 predictor, while trait negative affectivity and past-month PTSD symptom severity served as the level-2 predictors. Daily stress was person-centered, meaning that each woman's mean of daily stress ratings across the seven days was subtracted from each of her daily stress scores. In this way, the level-l intercept represented the predicted level of emotion for each woman when her daily stress on a given day is equal to her average level of daily stress (Schwartz \& Stone, 1998; Nezlek, 2001). All level-2 predictors were centered around their respective grand-means.

The level-1 equation (4), level-2 slope and intercept equations ( $5 \mathrm{a}$ and $5 \mathrm{~b}$ ), and the combined full model (6) for testing associations between PTSD symptom severity, daily stress, and daily emotion are specified below. In these equations, EMOTION represents daily negative or positive emotion intensity, while STRESS represents daily stress ratings, NA represents trait negative affectivity, and PTSD represents past-month PTSD symptom severity.

$$
\text { EMOTION }_{i j}=\beta_{0 j}+\beta_{1 j}\left(\operatorname{STRESS}_{i \mathrm{j}}\right)+r_{\mathrm{ij}}
$$




$$
\begin{aligned}
& \text { Intercept: } \beta_{0 \mathrm{j}}=\gamma_{00}+\gamma_{01}\left(\mathrm{NA}_{\mathrm{j}}\right)+\gamma_{02}\left(\mathrm{PTSD}_{\mathrm{j}}\right)+\mathrm{u}_{0 \mathrm{j}} \\
& \text { Slope: } \quad \beta_{1 \mathrm{j}}=\gamma_{10}+\gamma_{11}\left(\mathrm{NA}_{\mathrm{j}}\right)+\gamma_{12}\left(\mathrm{PTSD}_{\mathrm{j}}\right)+\mathrm{u}_{1 \mathrm{j}} \\
& \text { EMOTION }_{\mathrm{ij}}=\gamma_{00}+\gamma_{01}\left(\mathrm{NA}_{\mathrm{j}}\right)+\gamma_{02}\left(\mathrm{PTSD}_{\mathrm{j}}\right)+\gamma_{10}\left(\mathrm{STRESS}_{\mathrm{ij}}\right)+ \\
& \gamma_{11}\left(\mathrm{NA}_{\mathrm{j}}\right)\left(\mathrm{STRESS}_{\mathrm{ij}}\right)+\gamma_{12}\left(\mathrm{PTSD}_{\mathrm{j}}\right)\left(\mathrm{STRESS}_{\mathrm{ij}}\right) \\
& +\mathrm{u}_{0 \mathrm{j}}+\mathrm{u}_{1 \mathrm{j}}\left(\mathrm{STRESS}_{\mathrm{ij}}\right)+\mathrm{r}_{\mathrm{ij}}
\end{aligned}
$$

In the level-1 equation (4), the predicted level of daily emotion at signal $i$ for woman $j$ is modeled as a function of woman $j$ 's mean level of daily stress $\left(\beta_{0_{j}}\right)$, the relationship between daily stress and emotion for woman $j\left(\beta_{1 \mathrm{j}}\right)$, and a unique residual term for woman $j$ for signal $i$. In the level-2 intercept model (5a), woman $j$ s intercept is modeled as a function of the intercept for all women $\left(\gamma_{00}\right)$, the effects of trait negative affectivity $\left(\gamma_{01}\right)$ and past-month PTSD symptom severity $\left(\gamma_{02}\right)$, and the unique effect of woman $j$ on mean daily emotion. In the level-2 slope model (5b), woman $j$ 's slope is modeled as a function of the average relationship between daily stress and daily emotion (i.e., the average daily emotion-stress slope) when past-month PTSD symptom severity is equal to the grand mean $\left(\gamma_{10}\right)$ : the effects of trait negative affectivity $\left(\gamma_{11}\right)$ and past-month PTSD symptom severity $\left(\gamma_{12}\right)$ on daily stress-emotion slopes, and the unique effect of woman $j$ on the relationship between daily stress and daily emotion. Substituting equation (5a) and (5b) into equation (4) generates the full model, equation (6), in which daily emotion for woman $j$ at signal $i$ is modeled as a function of the overall sample mean $\left(\gamma_{00}\right)$, main effects of both within-persons (i.e., daily stress) and between-persons factors (i.e., trait negative affectivity and past-month PTSD symptom severity), as well as cross- 
level interactions representing relationships between within- and between-persons factors and both within- and between-persons error terms.

Hypotheses: Set 3

Hypothesis $3 a$. For testing within-persons associations between daily reexperiencing symptoms and daily negative emotion, one multilevel regression model was used. Severity of daily reexperiencing symptoms served as the dependent measure, intensity of daily negative emotion served as the level-1 predictor, and past-month severity of reexperiencing symptoms served as the level-2 predictor. Intensity level of daily negative emotion was person-centered so that the level-1 intercept represents the predicted level of negative emotion for each woman when her reexperiencing symptom severity on a given day is equal to her average daily reexperiencing symptom severity (Schwartz \& Stone, 1998; Nezlek, 2001), and past-month reexperiencing symptom severity was centered around the grand mean (Raudenbush \& Bryk, 2002; Nezlek, 2001). The level-1 equation (7), level-2 slope and intercept equations ( $8 \mathrm{a}$ and $8 b$ ), and the combined full model (9) for testing associations between daily reexperiencing symptoms and daily negative emotion are specified below. In these equations, REEXP represents severity of daily reexperiencing symptoms, NEG EMOTION represents intensity of daily negative emotion ratings, and PTSD REEXP represents past-month reexperiencing symptom severity.

$$
\operatorname{REEXP}_{\mathrm{ij}}=\beta_{0 \mathrm{i}}+\beta_{\mathrm{lj}}\left(\text { NEG EMOTION }_{\mathrm{ij}}\right)+\mathrm{r}_{\mathrm{ij}}
$$

Intercept: $\beta_{0 \mathrm{j}}=\gamma_{00}+\gamma_{01}\left(\right.$ PTSD REEXP $\left._{\mathrm{j}}\right)+\mathrm{u}_{0 \mathrm{j}}$ 
Slope: $\quad \beta_{1 \mathrm{j}}=\gamma_{10}+\gamma_{11}\left(\operatorname{PTSD~REEXP}_{\mathrm{j}}\right)+\mathrm{u}_{1 \mathrm{j}}$

$$
\begin{aligned}
\operatorname{REEXP}_{i \mathrm{j}}= & \gamma_{00}+\gamma_{01}\left(\text { PTSD REEXP }_{\mathrm{j}}\right)+\gamma_{10}\left(\text { NEG EMOTION }_{\mathrm{ij}}\right)+ \\
& \gamma_{11}\left(\text { PTSD REEXP }_{\mathrm{j}}\right)\left(\text { NEG EMOTION }_{\mathrm{ij}}\right)+\mathrm{u}_{0 \mathrm{j}}+ \\
& \mathrm{u}_{1 \mathrm{j}}\left(\text { NEG EMOTION }_{\mathrm{ij}}\right)+\mathrm{r}_{\mathrm{ij}}
\end{aligned}
$$

In the level-1 equation (7), the predicted severity of daily reexperiencing symptoms on day $i$ for woman $j$ is modeled as a function of woman $j$ 's mean level of daily negative emotion $\left(\beta_{0 \mathrm{j}}\right)$, the relationship between daily reexperiencing symptom severity and negative emotion for woman $j\left(\beta_{\mathrm{lj}}\right)$, and a unique residual term for woman $j$ on day $i$. In the level-2 intercept model ( $8 \mathrm{a})$, woman $j$ 's intercept is modeled as a function of the intercept for all women $\left(\gamma_{(0)}\right)$, the effects of past-month severity of reexperiencing symptoms $\left(\gamma_{01}\right)$, and the unique effect of woman $j$ on mean daily reexperiencing symptom severity while holding past-month reexperiencing symptom severity constant. In the level-2 slope model ( $8 \mathrm{~b}$ ), woman $j$ "s slope is modeled as a function of the average relationship between daily reexperiencing and daily negative emotion (i.e., the average daily reexperiencing symptoms severity-negative emotion slope) when past-month reexperiencing symptom severity is equal to the grand mean $\left(\gamma_{10}\right)$; the effect of pastmonth reexperiencing symptom severity $\left(\gamma_{11}\right)$ on daily reexperiencing-negative emotion slopes, and the unique effect of woman $j$ on the relationship between daily reexperiencing symptoms and daily negative emotion. Thus, substituting equation (8a) and (8b) into equation (7) generates the full model, equation (9), in which daily reexperiencing symptom severity for woman $j$ on day $i$ is modeled as a function of the overall sample 
mean $\left(\gamma_{00}\right)$, main effects of both within-persons (i.e., daily negative emotion) and between-persons factors (i.e., past-month reexperiencing symptom severity), as well as a cross-level interaction representing the relationship between the within- and betweenpersons factors and both within- and between-persons error terms.

Hypothesis $3 h$. For testing the within-persons temporal relationship between daily emotional numbing symptoms, daily reexperiencing symptoms, and daily negative emotion, a time-lagged multilevel model was used. Because the predictor is a variable temporally preceding the outcome variable, time-lagged analyses allow for some degree of causal inference with respect to associations between predictor and outcome variables (King et al., 2000; Bolton, Gray, \& Litz, 2006). The level-1 equation (10), level-2 slope and intercept equations ( 1 la-d), and the combined full model (12) for testing time-lagged associations between daily emotional numbing symptoms, daily reexperiencing symptoms, and daily negative emotion are specified below. In these equations, NUMB represents severity of daily numbing symptoms, REEXP represents severity of daily reexperiencing symptoms, and NEG EMOTION represents daily negative emotion. To

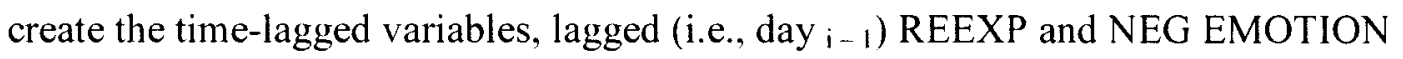
variables were first formed using the SAS LAG function, and the interaction term was then created from grand mean centered main effect REEXP and NEG EMOTION lagged variables and tested in its uncentered form (Nezlak, 2003).

$$
\begin{aligned}
\operatorname{NUMB}_{i j}= & \beta_{0 j}+\beta_{1 j}\left(\operatorname{REEXP}_{i-1 j}\right)+\beta_{2 j}\left(\text { NEG EMOTION }_{i-1 \mathrm{j}}\right)+ \\
& \beta_{3 j \mathrm{j}}\left(\text { REEXP }_{\mathrm{i}-1 \mathrm{j}}\right) \times\left(\text { NEG EMOTION }_{\mathrm{i}-1 \mathrm{j}}\right)+r_{i j}
\end{aligned}
$$




$$
\begin{array}{ll}
\text { Intercept: } & \beta_{0 \mathrm{j}}=\gamma_{00}+\mathrm{u}_{0 \mathrm{j}} \\
\text { Slope: } & \beta_{1 \mathrm{j}}=\gamma_{10}+\mathrm{u}_{1 \mathrm{j}} \\
& \mathrm{B}_{2 \mathrm{j}}=\gamma_{20}+\mathrm{u}_{2 \mathrm{j}} \\
\mathrm{B}_{3 \mathrm{j}}=\gamma_{30}+\mathrm{u}_{3 \mathrm{j}}
\end{array}
$$

$$
\begin{aligned}
& \text { NUMB }_{i j}=\gamma_{00}+\gamma_{10}\left(\operatorname{REEXP}_{\mathrm{i}-1 \mathrm{j}}\right)+\gamma_{20}\left(\text { NEG EMOTION }_{\mathrm{i}-1 \mathrm{j}}\right)+ \\
& \gamma_{30}\left[\left(\operatorname{REEXP}_{\mathrm{i}-1 \mathrm{j}}\right) \times\left(\mathrm{NEG} \mathrm{EMOTION}_{\mathrm{i}-\mathrm{ij}}\right)\right]+ \\
& u_{0 j}+u_{1 j}\left(\operatorname{REEXP}_{i-1 j}\right)+u_{2 j}\left(\text { NEG EMOTION }_{i-1 j}\right)+ \\
& u_{3 j}\left[\left(\operatorname{REEXP}_{i-1 j}\right) \times\left(\text { NEG EMOTION }_{i-1 j}\right)\right]+r_{i j}
\end{aligned}
$$

In the level-1 equation (10), the predicted severity of daily emotional numbing symptom severity on day $i$ for woman $j$ is modeled as a function of woman $j$ s mean level of daily numbing symptoms $\left(\beta_{0 \mathrm{j}}\right)$, main effects of reexperiencing symptoms $\left(\beta_{1 \mathrm{j}}\right)$ and negative emotion $\left(\beta_{2 \mathrm{j}}\right)$ on the preceding day $(i-1)$ for woman $j$, the relationship between numbing symptoms and the interaction between daily reexperiencing symptoms and negative emotion on the preceding day $(i-1)$ for woman $j\left(\beta_{3 \mathrm{j}}\right)$, and a unique residual term for woman $j$ on day $i$. Because no level-2 predictors are specified for hypothesis $3 \mathrm{~b}$, in the level-2 intercept model (11a), woman $j$ 's intercept is modeled simply as a function of the intercept for all women $\left(\gamma_{00}\right)$ and the unique effect of woman $j$ on mean daily numbing symptom severity. Likewise, in the level-2 slope models (11b-d), woman $j$ 's slope for each effect (i.e., main effect for lagged daily reexperiencing symptoms, main effect for lagged negative emotion, and the interaction between lagged daily reexperiencing symptoms and negative emotion) are modeled as a function of the average relationship, 
or slope, between daily numbing symptoms and the following effects: lagged reexperiencing symptoms $\left(\gamma_{10}\right)$; lagged negative emotion $\left(\gamma_{20}\right)$; and their interaction $\left(\gamma_{30}\right)$, as well as random effects, or residuals associated with mean daily numbing symptoms $\left(\mathrm{u}_{0 \mathrm{j}}\right)$ and the relationship between daily numbing symptoms and each effect (i.e., daily lagged reexperiencing symptoms $\left(\mathrm{u}_{1 \mathrm{j}}\right)$; daily lagged negative emotion $\left(\mathrm{u}_{2 \mathrm{j}}\right)$; and the interaction between daily reexperiencing symptoms and negative emotion $\left.\left(u_{3_{j}}\right)\right)$ as well as a unique level-1 residual term for woman $j$ on day $i$. Thus, in the full model (12), daily numbing symptom severity for woman $j$ on day $i$ is modeled as a function of the overall sample mean $\left(\gamma_{00}\right)$, within-day lagged reexperiencing and negative emotion main effects, the interactive effect of within-person daily reexperiencing symptoms and daily negative emotion on the preceding day, and within-and between-persons error terms. 


\section{RESULTS}

\section{Compliance with Experience Sampling Procedures}

Although PDAs were programmed to signal 49 times over the course of the week (i.e., 7 signals each day), in actuality, there was variability among participants in the number of signals received each day. This is suspected to be related to study initiation time by the participant and response latency to the PDA when signaled; however, this was not a problem that surfaced during piloting of and calibrating the Palm Pilots prior to enrolling participants, and thus this hypothesis could not readily be examined. Total number of signals received by participants over the course of the study ranged from 24 to 49 , with women receiving a total of 44 signals on average $(S D=4.76)$. Women completed an average of $34(S D=7.46)$ diary ratings over the course of their participation (range $=18-45)$, or $77.40 \%$ of signals on average $($ range $=36.73 \%$ to $97.83 \%)$.

\section{Calculating Proportion of Missing Electronic Diary Data}

Due to variability in the number of signals received among women, proportion of missing data was calculated by dividing the number of missed signals as recorded by the PDA by the total number of signals received by a participant (e.g., 7 missed signals / 49 total signals received $=14.28 \%$ of the total number of signals missed; 7 missed signals $/$ 42 total received $=16.67 \%$ of the total number of signals missed). As previously mentioned, one woman ended participation in the present study after five days citing 
increased time restrictions in her daily schedule. For this participant, proportion of missing data was calculated only for those days completed. Likewise, two women experienced PDA malfunctions which caused them to miss completing one full day of assessments for the study. Thus, for these participants, proportion of missing data was calculated for signals completed in those six days rather than the full seven days.

Daily Emotion Assessments. Of the 38 women who enrolled and provided diary data for the present study, 30 women missed $30 \%$ or fewer emotion diary ratings (i.e., proportion of missing data was equal to or less than $30 \%$ of the total number signals). More specifically, 20 women had $20 \%$ or fewer missing assessments, six women had 21 $25 \%$ assessments missing, and four women had 26-30\% assessments missing. Eight women had nearly $40 \%$ or more of emotion diary ratings missing (i.e., $36-63 \%$ of the total assessments missing).

A major aim of the present study was to capture daily experiences as accurately as possible among women exposed to prior IPV. One advantage of multilevel modeling approaches compared to other statistical approaches is their ability to handle unbalanced designs (i.e., cases with missing level-1 data). However, for women with a substantial amount of missing data, it could not be safely assumed that their data were missing at random or that their assessments represented an accurate sampling of their daily experiences. Review of some extant diary studies suggests exclusion of participants if more than $20 \%$ of data are missing (e.g., Eaton \& Funder, 2001; Stone \& Shiffman, 2002); however, others note the absence of absolute rules and recommend examination of potential patterns of missing data as part of the decision-making process (e.g., Tabachnick \& Fidell, 2007; Nezlak, 2003). Based on these suggestions along with 
consideration of study goals and statistical power, while at the same time attempting to maximize generalizability of findings by not being overly exclusive on the basis of missing data, a participant was excluded from relevant analyses in the present study if more than $30 \%$ of her total assessments were missing. Therefore, for the purposes of describing characteristics of daily emotional experiences (i.e., Aim 1 of the present study), and for testing hypotheses $1 \mathrm{a}$ and $1 \mathrm{~b}$, the final subsample included 30 women with a total of 1090 completed emotion assessments across the subsample. Of note, among this subsample of 30 women, $84 \%$ of emotion assessments were completed on average, a rate comparable to that reported in similar diary-based studies (e.g., $85 \%$; Trull \& Ebner-Priemer, 2009).

Daily PTSD and Daily Stress Assessments. Because women had the option of completing paper PCL questionnaires if the final signal of the day was missed, proportion of missing daily PTSD and daily stress ratings were calculated separately from proportion of missing daily emotion assessments. In this case, proportion of missing daily PTSD and stress assessments was calculated by dividing the number of missed assessments by the total number of assessments (i.e., 7 for all participants). Of the 38 women who enrolled and provided diary data for the present study, 31 women missed two or fewer daily PTSD and stress assessments (i.e., fewer than $30 \%$ of total assessments), with 16 women completing all seven daily PTSD and stress assessments. Seven women missed three or more daily PTSD and stress assessments (i.e., $43-86 \%$ of total assessments) and thus were excluded from relevant analyses (i.e., testing hypotheses $2 \mathrm{a}$ and $\mathrm{b}$ and hypotheses $3 \mathrm{a}$ and $\mathrm{b}$ ); however, this included four women who were already excluded on 
the basis of missing emotion assessments. Thus, for the purposes of testing hypotheses $2 \mathrm{a}$ and $2 \mathrm{~b}$ and hypotheses $3 \mathrm{a}$ and $3 \mathrm{~b}$, the final subsample included 27 women.

\section{Data Cleaning and Screening Procedures}

Prior to descriptive and statistical analyses, level-1 and level-2 variables were examined using SAS PROC MEANS and SAS PROC UNIVARIATE to ascertain accuracy of data entry, restructuring, and reduction procedures by indentifying any outof-range values; to identify potential outliers; and to inspect the distribution characteristics of variables to be used in analyses. Possible associations between noncompliance (i.e., exclusion based on missing diary data) and relevant level-2 characteristics as well as potential patterns among missing level-1 data were also examined (Tabachnick \& Fidell, 2007).

First, level-1 and level-2 variables were standardized and resulting z-scores were inspected for potential outliers (i.e., z score greater than 3.29, p $<.001$; Tabachnick \& Fidell, 2007). Given the relatively larger sample size with respect to assessments or signals, a small number of extreme values was expected for some participants on level-1 variables; however, based on the above conventions, one participant had a large number of extreme daily negative emotion scores (i.e., 13) and as such, was identified as a potential outlier. As a result, initial analyses were run with and without this participant's data. Because the patterns of results did not differ appreciably between analyses, the reported results reflect inclusion of this participant's data. No participants were identified as outliers with respect to level-2 variables. With regard to variable distribution, inspection of plots indicated a positively skewed distribution for daily negative emotion with positive kurtosis. Because violations of normality may result in 
biased estimates in multilevel modeling approaches (Raudenbush \& Bryk, 2002), negative emotion values were log-transformed in an attempt to approximate normality. Although this transformation improved skewness and kurtosis slightly, findings did not appreciably differ when modeling using transformed scores versus non-transformed scores. Thus, a decision was made to use non-transformed data for ease of interpretation.

Next, analyses were run to determine whether there may be associations between non-compliance, or exclusion based on incomplete daily diary data, and relevant level-2 characteristics (i.e., past-month PTSD symptom level and trait negative affectivity). With respect to daily emotion assessments, there were no significant differences between compliant women and non-compliant women (i.e., women excluded due to missing data) with respect to either mean level of past-month PTSD symptoms $(33.87$ vs. $33.00 ; t(36)=$ $-.024, p=.82)$ or trait negative affectivity (i.e., neuroticism) scores $(2.82$ vs. $2.96 ; t(36)=$ $.85, p=.40$ ). With respect to daily PTSD symptom and stress assessments, there were no significant differences in past-month PTSD scores between non-compliant women and compliant women $(38.14$ vs. $32.68 ; t(36)=1.23, p=.25)$. Likewise, mean difference in trait negative affectivity scores were not significant between non-compliant and compliant women $(2.82$ vs. $2.85 ; t(36)=-.19, p=.85)$.

Finally, potential patterns in missing level-1 data were inspected among women included for analyses. Some caution that cases with missing data may distort findings if there are discernable patterns underlying missingness, which in turn, are associated with other measured factors (e.g., Tabachnick \& Fidell, 2007). Given this, potential patterns in missingness were examined in order to increase confidence that missing data of all included women were more likely missing at random than not. For each of the 30 
participants, three possible patterns among missing daily emotion data were examined: missing more than $50 \%$ of first-of-the-day signals (i.e., were there any participants who had more than $50 \%$ of all first signals missing?); missing more than $50 \%$ of end-of-theday signals; and whether $50 \%$ of signals were missing for any participant on any given day. However, none of these patterns were observed among any the 30 women included in analyses.

\section{Participant Characteristics}

Information presented in the text below describes the total sample of 38 women who provided diary data for the present study. Table 1 presents characteristics for the total sample, for each of the subsamples used in analyses as described above, and for those women excluded from each subsample.

\section{Sociodemographic Characteristics}

The sample was mostly European American or white with an average age of 55 years and a range of 46 to 61 years. Most women were employed full-time, and on the whole, women were highly educated, with over half of the sample completing a college degree or graduate or professional training. The majority of women described their marital status as "divorced" (i.e., not currently married or partnered), and nearly twothirds reported a household income of $\$ 40,000$ or less annually.

\section{Between-persons (Level-2) Characteristics}

With respect to prior experiences of partner violence, all but two women endorsed at least one of 12 items reflecting acts of physical violence, with $87 \%(n=33)$ of the total sample endorsing at least one act classified as severe. In general, women`s responses indicated chronic exposure to severe violence. That is, the average number of severe 
physically violent acts was $18(S D=28.82)$ across the sample, while only two women endorsed experiencing only one act of severe physical violence in past intimate relationships. Furthermore, all women who endorsed at least one act of severe physical violence also endorsed sustaining injuries from such acts, with nearly $60 \%$ endorsing injuries also classified as severe (e.g., needed or actually received medical attention). Additionally, $55 \%(n=21)$ of the total sample endorsed at least one of four items reflecting acts of severe sexual coercion or violence. Women's responses suggest that exposure to severe sexual violence was also chronic in that the average number of acts of severe sexual coercion or violence endorsed was $14(S D=19.44)$.

With regard to PTSD symptoms, mean total past-month PTSD symptom severity score was $33.87(S D=10.98)$ with a range of 19 to 68 . Conventionally, cut score recommendations for identifying probable PTSD cases based on different versions of the PCL have ranged from scores of 44 to 50 ; however, these samples have primarily consisted of men and individuals exposed to acute trauma (e.g., Norris \& Hamblen, 2004). More recent data from primary care samples of female veterans (Lang, Laffaye, Satz, Dresselhaus, \& Stein, 2003) and women enrolled in an HMO (Walker, Newman, Dobie, Ciechanowski, \& Katon, 2002) suggest a PCL-C total score of 30 or higher as optimal for identifying women with probable PTSD outside of acute trauma settings. In the two samples cited above, mean total PCL-C scores were 39 and 27 , respectively, which were similar overall to that found in the present study. Although PCL-C scores were used in analyses as a continuous measure of PTSD symptom severity, using the suggested cut score of 30 , nearly two-thirds of the present sample $(n=19)$ were identified as probable PTSD cases based on their PCL-C responses. Of particular interest to the 
present study, $80 \%$ of women endorsed being bothered by intrusive reexperiencing symptoms in the form of thoughts, memories, or images of a stressful past event; twothirds of women endorsed feeling upset at reminders of stressful past event; and $43 \%$ endorsed feeling emotionally numb over the past month, rates comparable to those reported by women with IPV histories in a previous study (Woods, 2000).

With respect to trait negative affectivity, scores on the BFI neuroticism scale ranged from 1 to 4.13 , while the average score was $2.85(S D=.69)$. This is comparable to normative data obtained from a large sample of similarly-aged women in that the mean neuroticism score for women aged 55 years was 2.93 (S.D. Gosling, personal communication, January 13, 2010).

\section{Aim 1: Characteristics of Daily Emotional Experiences}

The first aim of the present study was to provide a characterization of women's emotional experiences as well as the contexts in which these emotions occur. To accomplish this. several aspects of women's daily discrete emotional experiences were examined. These included aspects related to the occurrence and frequency of specific emotions, the intensity at which specific emotions occurred, and the variability of specific emotions within and across days. The activities and social context surrounding women's emotional experiences were also explored.

\section{Occurrence and Frequency of Specific Emotions}

First, to gain a broad picture of women's emotional experiences, the number of women endorsing the occurrence of a given emotion at any time during the sampling period was examined. In essence, this was motivated by the following question: did all women experience each emotion at some point, or were there emotions that women never 
or very rarely experienced during the course of their participation? In this case, occurrence for a given emotion is defined as an intensity rating greater than 1 at any time during the sampling frame (e.g., Schimmack \& Diener, 1997). With respect to positive emotions, all 30 women ( $100 \%)$ endorsed joy and interest at some point during the sampling period as well as surprise. With regard to negative emotions, 28 women $(93.33 \%)$ endorsed anger; 27 women endorsed contempt $(90 \%) ; 26$ endorsed sadness $(86.67 \%) ; 23$ women $(76.67 \%)$ each endorsed guilt and disgust; 20 women $(66.67 \%)$ endorsed fear; and 18 women $(60 \%)$ endorsed shame at some point during their course of their participation. Taken together, this suggests that women experienced a range of emotions, both positive and negative, during the course of their participation.

The frequency with which specific emotions occurred over the course of the sampling period was examined next. In this case, the frequency of a given emotion was computed as the proportion of non-missing assessments in which that emotion received an intensity rating greater than 1, averaged across women (e.g., Zelenski \& Larsen, 2000). Mean frequencies for each emotion are presented in Table 2. In general, positive emotions were more frequently endorsed than were negative emotions. That is, while joy and interest were endorsed by women on $99 \%$ and $97 \%$ of occasions, respectively, endorsement of negative emotions ranged from $37 \%$ (i.e., sadness) to $19 \%$ (i.e., fear). Frequency of specific emotions was also examined at the level of the individual in order to determine which emotions were most prevalent, or most frequently occurring, for each individual woman. For each emotion, this was computed as the number of women who rated a given emotion's intensity greater than 1 more frequently than all other emotions. For the majority of women, positive emotions were by far the most frequently occurring, 
with joy endorsed most frequently by 5 women (16.67\%), interest most frequently by 5 women $(16.67 \%)$, and joy and interest with equal frequency for 16 women $(50.33 \%)$. Three women experienced negative emotions most frequently. That is, contempt was endorsed most frequently by one woman $(3.33 \%)$; contempt and fear were equally frequent for one woman; and guilt and shame occurred with equal frequency for one woman. Only one woman endorsed both positive and negative emotions with equal frequency (i.e., joy, interest, sadness, anger, contempt, fear, guilt, shame, and surprise). Intensity of Specific Emotions

Mean intensity ratings for each emotion across all subjects are presented in Table 2. In this case, emotion non-occurrence was controlled for by excluding emotion ratings equal to 1 so that mean intensity values reflect how intensely, on average, an emotion was experienced when the emotion occurred (Schimmack \& Diener, 1997; Zelenski \& Larsen, 2000). Interest was rated with more intensity than were other emotions $(M=$ 3.62), while joy received similar ratings $(M=3.58)$. Negative emotions were rated with less intensity than were positive emotions and had mean intensity rating values that fell below 3.00, although exceptions included contempt $(M=3.34)$ and disgust $(M=3.00)$. Additionally, mean intensity of specific emotions was examined at the level of the individual in order to determine which emotions were experienced most intensely on average for each woman. In this instance, fourteen women rated joy most intensely $(46.67 \%), 11$ women $(36.67 \%)$ rated interest most intensely, three women $(10 \%)$ rated contempt most intensely, and two women $(6.67 \%)$ rated guilt most intensely. Intraindividual Variability of Specific Emotions 
In addition to frequency and mean intensity of specific emotions, the variability of emotions among individual women was examined. Although different methods have been used to measure intraindividual variability, it is frequently measured as the withinperson standard deviation (Ed \& Diener, 1999), and as such, is interpretable as a measure of the overall stability of a given emotion over a period of time (e.g., Kashdan, Uswatte, et al., 2006). For the present study, intraindividual variation was calculated as the withinperson standard deviation of a given emotion`s ratings for each day. These standard deviation scores were then averaged across days and across women to create mean within-person standard deviation scores for each emotion, which are presented in Table 2. In general, while positive emotions were experienced with greater frequency and greater intensity on the whole than were negative emotions, this was not entirely the case with respect to intraindividual variability. That is, while most negative emotions exhibited less variation across ratings than did positive emotions, with mean within-person standard deviations ranging from .16 (shame) to .37 (contempt), both sadness $(M=.59)$ and anger $(M=.66)$ exhibited similar variation in ratings to that of joy $(M=.54)$ and interest $(M=$ .67). In other words, women's experiences of sadness and anger varied more widely between rating occasions than did their experiences of other negative emotions. Also, while women experienced joy and interest quite frequently, these emotional experiences were also characterized by greater changes in levels between rating occasions.

\section{Context of Emotional Experiences}

Finally, to provide further characterization of women's emotional experiences, the social contexts and activities surrounding women's emotion ratings were examined. With respect to social context, women endorsed being with others (i.e., family, co- 
workers, strangers or acquaintances, friends, or significant other or spouse) on approximately half of the reporting occasions (50.44\%) and being alone or with pets on approximately half of the reporting occasions (49.56\%). It was of interest to know whether specific emotions occurred with more or less frequency in different social contexts, and to simplify this examination, social context was characterized as either "social" (i.e., occasions in which women endorsed being with other people) or "nonsocial" (i.e., occasions in which women endorsed being alone or with pets). Only the emotions of fear and shame showed frequencies that differed noticeably by social context, with frequencies of these two emotions relatively greater in social, as opposed to non-social context. See Table 3.

With respect to activities surrounding their emotional experiences, women endorsed task-oriented activities (i.e., working at a job or running errands/doing chores) on nearly half of reporting occasions (45.44\%). Leisure activities or resting and socializing were endorsed on $33.75 \%$ and $10.64 \%$ of occasions, respectively, while endorsement of "other" activities not listed accounted for $10.18 \%$ of rating occasions. Considering the frequency of specific emotions across activity contexts, anger and shame occurred with the greatest frequency on those occasions in which women endorsed working at a job/running errands; guilt and contempt occurred with the greatest frequency on those occasions in which women endorsed socializing, and sadness, disgust, and fear were endorsed on those occasions in which women endorsed "other" activities not listed. Both joy and interest were endorsed at equally high frequencies across all activity contexts.

Aim 1: Characteristics of Daily PTSD Symptoms and Experiences of Stress 
Also in line with Aim 1, it was of interest to provide a characterization of women's daily experiences of stress and symptoms of PTSD. Similar to examinations of daily emotional experiences, these examinations included the intensity or severity of daily PTSD symptoms and daily stress, the frequency at which PTSD symptoms occurred, and the variability of PTSD symptoms within and across days. See Table 2. Averaging across the sampling period and across women, mean daily PTSD symptom severity score was $29.50(S D=11.65)$, with a range of 16 to 72 . Compared to mean past-month PTSD symptom severity, mean daily PTSD symptom severity was slightly lower. With respect to individual symptom clusters, average symptom severity was highest for daily avoidance symptoms $(M=11.84, S D=5.33)$ and lowest for daily reexperiencing symptoms $(M=8.32, S D=4.07)$. Daily stress ratings averaged 3.71 ( $S D$ $=2.41)$ across the sampling period, with a range of 0 to 9 .

With respect to frequency of daily PTSD symptom occurrence, a symptom was considered to have occurred if the corresponding symptom item received a severity rating greater than 1, and a cluster of symptoms was considered to have occurred if any symptom from that cluster was endorsed. Over the course of the sampling period, hyperarousal symptoms were the most frequently occurring, with endorsement on $69.31 \%$ of rating occasions. Furthermore, among hypersarousal symptoms, difficulty concentrating was the most likely to be endorsed $(69.64 \%)$, while hypervigilance was the least likely to be endorsed (39.52\%). Avoidance symptoms were endorsed on $60.32 \%$ of occasions over the course of the sampling period. Feeling distant or cut off from other people was the most frequently occurring avoidance symptom (53.89\%), although loss of interest in activities and avoiding thinking, talking, or having feelings about a past event 
were endorsed at similar frequencies $(51.79 \%$ and $51.19 \%$, respectively). Trouble remembering parts of a past stressful event was the least frequently endorsed avoidance symptom $(27.71 \%)$. Reexperiencing symptoms were endorsed on $55.03 \%$ of all rating occasions. Feeling upset at reminders of a past event $(55.36 \%)$ and unwanted memories $(51.19 \%)$ were among the reexperiencing symptoms most frequently endorsed, while upsetting dreams of a past event were the least frequently endorsed symptom $(25.60 \%)$. Intraindividual variability of daily PTSD symptoms and experiences of stress were calculated using within-person standard deviations across the seven days. However, it should be noted that, because PTSD symptoms and daily stress were assessed only once per day, intraindividual variability of daily symptoms reflects variation in women"s symptom severity ratings across days only (i.e., not within and across days, such as that for emotion ratings). Mean within-person standard deviation for daily stress scores was $1.20(S D=.99)$. Mean within-person standard deviation values for total PTSD symptom severity scores was $5.43(S D=3.69)$, which suggests that, on the whole, women did experience measurable, day-to-day fluctuations in PTSD symptoms over the course of the week. Considering symptom clusters, intraindividual variability values were similar for reexperiencing symptoms $(2.23, S D=1.58)$ and avoidance symptoms $(2.35, S D=1.62)$, while hyperarousal symptoms exhibited somewhat less variation on average $(1.76, S D=$ 1.23). With respect to individual symptoms, within-person standard deviations ranged from .29 (i.e., sense of foreshortened future) to .68 (i.e., feeling very upset at reminders of a past stressful event). Thus, when considering all individual symptoms together, women experienced on average the greatest variability, or wider day-to-day fluctuations, in emotional reactivity to reminders of a past stressful event. 


\section{Statistical Tests of Hypotheses}

Hypotheses: Set 1

The first set of hypotheses predicted that, after accounting for the effects of trait negative affectivity, women with higher past-month PTSD symptom severity would evidence greater frequency and greater intensity of negative emotion and lower frequency and intensity of positive emotion. These associations were tested using four ordinary least squares hierarchical linear regression models with predictors centered around their respective grand means. After adjusting for trait negative affectivity, the effect of pastmonth PTSD symptom severity was significant for frequency of negative emotion $t(27)=$ $2.88, B=.01, p=.008$, but not intensity of negative emotion $(p=.39)$. There was no significant effect of past-month PTSD symptom severity on either positive emotion frequency or intensity $(p s>.55)$. Trait negative affectivity was significantly and negatively associated with intensity of positive emotion, $t(27)=-2.15, B=-.31, p=.04$, but not frequency of positive emotion $(p=.13)$

Effects of past-month PTSD symptom severity on levels of daily negative emotion and daily positive emotion levels were also modeled using multilevel random coefficients regression. Unconditional models (i.e., intercept-only models with no level-2 predictors) were run first in order to determine whether there was a significant amount of variance in emotion intercepts (i.e., mean negative and positive emotion levels) to be explained, and if so, to determine the variance in emotion levels that could be attributed to between-persons factors as opposed to within-persons factors. Results of these unconditional models indicated a significant amount of variance in both negative emotion intercepts and positive emotion intercepts among women $(p s<.001)$. Calculation of the 
intraclass correlation coefficient indicated that $59 \%$ of this variability in negative emotion levels could be accounted for by between-persons factors, while $41 \%$ could be accounted for by within-persons factors. The corresponding values for positive emotions levels were $44 \%$ and $56 \%$, respectively. Thus, adding predictors at both levels was warranted for modeling both negative and positive emotion levels.

In line with hypotheses $1 \mathrm{a}$ and $\mathrm{lb}$, between-subjects variance in emotion levels was first modeled as a function of past-month PTSD symptom severity and trait negative affectivity, and results from these analyses are presented in Table 4. A significant fixed effect of past-month PTSD on negative emotion levels was found after accounting for the effect of trait negative affectivity, $\gamma_{12}=.023, t(27)=2.70, p=.001$. Comparison with the unconditional model indicated that variance in negative emotion intercepts was reduced by approximately $34 \%$ with the addition of trait negative affectivity and past-month PTSD, or that these level-2 predictors accounted for $30 \%$ of between-persons variance in mean negative emotion levels. For positive emotion levels, the effect of past-month PTSD was not significant after adjusting for trait negative affectivity, $\gamma_{02}=-.014, t(27)=$ $-1.43, p=.163$. With regard to positive emotion, although comparison with the unconditional model indicated that approximately $33 \%$ of the variance in positive emotion intercepts was accounted for by the addition of both predictors, this reduction in error variance was largely attributable to the significant negative effect of trait negative affectivity on positive emotion, $\gamma_{01}=-.36, t(27)=-2.62, p=.014$. Thus, while women with more severe past-month PTSD symptoms experienced, on average, higher levels of negative emotion during the week, PTSD symptoms did not account for differing levels of positive emotion observed among women. Rather, differences in positive emotion 
levels were better accounted for by trait negative affectivity. Examination of variance component estimates from both negative and positive emotion models indicated that a significant amount of variation in mean emotion levels remained to be explained. That is, even after the addition of level-2 predictors, both mean negative and positive emotion levels continue to exhibit significant variation between women.

Hypotheses: Set 2

These analyses attempted to model within-persons variance among daily emotion levels with the addition of daily stress as a level-1 predictor. Specifically, it was hypothesized that after accounting for effects of trait negative affectivity, the positive association between negative emotion and stress would be greater among women with more severe past-month PTSD. Likewise, a negative association between positive emotion and stress was expected with greater negative associations among women with more severe past-month PTSD. Negative and positive emotion levels were first modeled as a function of stress without the inclusion of level-2 predictors. Results from these models indicated that mean negative emotion-stress slope (i.e., the mean relationship between negative emotion and stress averaged across all women) differed significantly from zero, or that stress and negative emotion were indeed significantly and positively related. More specifically, this relationship was characterized by an average .095 increase in negative emotion ratings for every 1 -point increase in stress ratings, $\gamma_{10}=$ $.095, t(26)=3.25, p=.004$. For positive emotion, results indicated a significant, but negative, relationship with stress ratings, which was characterized by a decrease of .064 in positive emotion ratings for every 1 -point increase in stress on average, $\gamma_{10}=-.064$, $t(26)=-2.81, p=.010$. 
Comparison with the model containing only stress as the predictor revealed that inclusion of level- 2 variables accounted for $23 \%$ of between-subjects variance in emotion-stress slopes. After controlling for trait negative affectivity, past-month PTSD severity emerged as a significant moderator of the relationship between negative emotion and stress, $\gamma_{12}=.009, t(24)=2.15, p=.042$. See Table 5. This cross-level interaction was probed using simple slopes analysis, a technique applicable to multilevel regression (Preacher, Curran, \& Bauer, 2006). In this instance, "high" and "low" past-month PTSD were operationalized as one standard deviation above and one standard deviation below the grand mean, respectively (Aiken \& West, 1991; Preacher et al., 2006). Examination of simple slopes indicated that, compared to women with lower PTSD symptom severity, women with higher PTSD symptom severity not only had higher negative emotion ratings overall, but as daily stress levels increased, their negative emotion ratings also increased significantly at a greater rate. In other words, although higher levels of stress were associated with greater negative emotion ratings in general, the effect of stress on negative emotion was significantly greater for women with greater past-month PTSD symptom severity, suggesting that these women were more stress-reactive than women with less severe past-month PTSD symptoms. See Figure 1. With respect to positive emotion, comparison with the model containing only stress as the predictor revealed that inclusion of level-2 variables accounted for approximately $12 \%$ of between-subjects variance in positive emotion ratings; however, neither trait negative affectivity nor pastmonth PTSD were significant factors in accounting for the relationship between positive emotion and stress $(p s>.44)$. See Table 5.

Hypotheses Set 3 
Hypothesis 3 a tested within-day associations between daily negative emotion and daily reexperiencing symptoms after accounting for past-month reexperiencing symptoms. The unconditional model (i.e., intercept-only, no level-1 or level-2 predictors) indicated significant variation in daily reexperiencing symptoms $(p<.001)$, while calculation of the interclass correlation coefficient indicated that $47 \%$ and $53 \%$ of variance was attributable to between-persons and within-persons factors, respectively. Daily reexperiencing symptoms were then modeled as a function of negative emotion and past-month reexperiencing symptoms. Results revealed no significant effect of reexperiencing-negative emotion slopes $(p=.184)$. A significant fixed effect was found for past-month reexperiencing symptom severity when included in level-2 models, but only for the intercept model, $\gamma_{01}=.412, t(25)=2.39, p=.025$. Furthermore, this effect corresponded to a reduction of $17 \%$ of between-persons variance in mean daily reexperiencing symptoms versus only a 5\% reduction in between-persons variance in daily reexperiencing-negative emotion slopes.

As a nonsignificant relationship between daily reexperiencing symptoms and negative emotion was unexpected, analyses were repeated using the full range of data available, rather than conducting within-day analyses by aggregating negative emotion ratings across day. This was based on the thought that aggregation of negative emotion scores across day could have possibly obscured the relationship between reexperiencing symptoms and emotion via loss of emotion rating variability or resulted in a loss of power to detect such a relationship due to fewer numbers of emotion ratings. However, the pattern of findings was not changed in that average daily reexperiencing symptomnegative emotion slope was also nonsignificant in this model $(p=.099)$. 
Hypothesis $3 \mathrm{~b}$ tested temporal associations between avoidance symptoms, and specifically emotional numbing symptoms, using a cross-lagged term which represented the interaction of previous day reexperiencing symptoms and negative emotion. It was predicted that emotional numbing symptoms on any given day would be greatest among women who experienced high levels of reexperiencing symptoms and high levels of negative emotion on the previous day. Results from the unconditional model indicated a significant amount of variance in emotional numbing symptom intercepts $(p<.001)$, while calculation of the intraclass correlation coefficient indicated that $69 \%$ and $31 \%$ of variance was attributable to between-persons and within-persons factors, respectively. Within-persons variance in numbing symptoms was then modeled as a function of the level-1 main effects of previous day reexperiencing symptoms and negative emotion as well as their interactive effect. Results indicated that the reexperiencing $\mathrm{x}$ negative emotion interaction slope was significant, $\gamma_{13}=-.469, t(26)=-3.925, p=.001$ as predicted. See Table 6. Furthermore, comparison of variance components from the unconditional and full model models indicated that addition of cross-lagged level-1 predictors accounted for $40 \%$ of within-persons variance in daily emotional numbing symptoms. The nature of this interaction was followed up using simple slopes analysis, as described previously; however, examination of simple slopes indicated a different pattern of findings than what was expected. Specifically, women with the highest levels of predicted numbing symptoms on a given day were those with high levels of reexperiencing symptoms but low levels of negative emotion on the previous day. In contrast, women with high levels of reexperiencing symptoms but high levels of negative emotion evidenced the lowest levels of predicted numbing symptoms on the following 
day. See Figure 2. Statistical significance testing of simple slopes (i.e., whether slopes differed significantly from zero) revealed that both the high reexperiencing slope $(t(128)$ $=4.04, p<.001)$ and low reexperiencing slope $(t(128)=2.46, p=.02)$ were significant. 


\section{DISCUSSION}

Although disruptions in emotional processes have been consistently identified as key features of PTSD, the exact nature of these disruptions is not well understood. The present study sought to advance understanding of emotion and PTSD by examining experiences of emotion, stress, and symptoms of PTSD as they unfolded in the course of everyday life among women with prior exposure to IPV. In doing so, the study had four major aims and expanded existing literature on PTSD and emotion in several ways. First, this study provided an ecologically valid characterization of women`s specific emotional experiences through the use of experiencing sampling methodologies and electronic

diaries. By focusing on women with prior exposure to IPV, the present study is the first, to this author`s knowledge, to provide naturalistic data on the everyday emotional experiences of IPV-exposed women, individuals who are at risk for chronic PTSD symptoms (Woods, 2000) and the disrupted emotional processes that accompany PTSD. Second, although some experience sampling studies have examined associations between PTSD and emotions in daily life, these studies have not to date included women, with the recent exception of Newton and Ho (2008), or accounted for possible effects of daily stress on the relationship between PTSD and daily emotion as in the present study. Finally, through assessment of daily PTSD symptoms, the present study was able to examine temporal associations between the experience of specific PTSD symptoms and daily emotion. More specifically, the study design allowed for empirical testing of 
theoretical propositions linking specific PTSD symptoms to emotional disruptions and allowed for some degree of causal inference regarding the relationship of daily PTSD symptoms to emotional experiences, an area which, to this author's knowledge, has not yet been explored.

\section{Hypotheses Set 1: Is PTSD Related to Women 's Daily Experiences of Emotion?}

Given the dearth of research in this area, one major aim of the present study was to expand on existing literature by testing associations between daily emotions and pastmonth PTSD symptom severity among women. In this regard, partial support was found for hypothesized associations as PTSD was associated with daily negative, but not positive, emotion levels.

\section{Negative Emotion}

The positive association between PTSD and daily negative emotion levels found in the present study adds to the growing body of empirical data supporting the "hyperemotionality" described in theories of emotional functioning in PTSD (e.g., Litz, 1992: Litz et al., 2000; Litz \& Gray, 2002). That is, although it is important to note that there have been a few exceptions, as a whole, cross-sectional questionnaire, laboratory, and experience sampling studies have demonstrated greater state and trauma-related negative emotions, greater negative emotion in response to emotional stimuli, and greater negative emotion in daily life, respectively, among individuals with PTSD.

With respect to theories of emotional disruptions in PTSD, it is proposed that PTSD results in more frequent and intense negative emotion due to the activation of trauma memory networks and associated negative emotional responses (e.g., Foa \& Rothbaum, 1998). From this, it may be presumed that greater PTSD symptom severity 
reflects more complex trauma memory networks and thus, more numerous and generalized associations with negative emotional responses. More severe PTSD could be expected to, in turn, lead to more frequent activation of trauma memory networks and thus, more frequent activation of negative emotion. In this regard, the significant and positive association between past-month PTSD symptom severity and negative emotion frequency observed in the present study offers support for this idea. However, contrary to theory and to this author`s expectation, past-month PTSD symptom severity was not associated with greater intensity of negative emotion. Rather, findings suggest that, while, on average, negative emotions did occur more frequently among women with more severe PTSD symptoms, once negative emotion was activated, it was not experienced any more intensely.

Importantly, the association between past-month PTSD symptom severity and daily negative emotion levels, and more specifically the association between past-month PTSD symptom severity and daily emotion frequency, held after accounting for the effects of trait negative affectivity. This suggests that the heightened negative emotion experienced by individuals with PTSD symptoms cannot wholly be attributed to an underlying predisposition to experience negative emotions in general, as studies of trait emotion and PTSD might suggest. That is, although it may be that higher levels of trait negative affectivity, or neuroticism, place an individual at risk for the development of PTSD, as some suggest (e.g., Cox et al., 2004), trait negative affectivity itself does not appear to account entirely for the emotional difficulties experienced by individuals following the development of PTSD symptoms. This points to the likelihood of other 
mechanisms underlying emotional difficulties which are specific to the experience of PTSD symptoms.

Why would PTSD be associated with greater frequency, but not intensity, of negative emotion? One possible explanation is simply that that the disruptions in emotional processes associated with PTSD may indeed be best characterized by more frequent occurrence, but not more intense experiences, of negative emotions. Given that the majority of naturalistic studies of PTSD and emotion (i.e., Muraoka et al., 1998; Beckham et al., 2000; Kashdan, Uswatte, et al. 2006; Kashdan, Julian et al., 2006) have not explicitly distinguished frequency from intensity in their assessment or analyses of emotional experiences, it seems possible that these different facets of emotional experiences may be blurred in existing studies. That is, it may be that the reported associations between greater negative emotion and PTSD in these studies in actuality reflect more frequent occurrence of negative emotion rather than intensity.

Some support for distinguishing between these facets of emotional experiences is provided by Newton \& Ho (2008), a study of ambulatory blood pressure which examined the emotional experiences of victimized women over the course of one day via paperand-pencil diaries. In direct contrast to the present study's findings, they found no association between PTSD symptom severity and occurrence of any of the negative emotions or negative states measured (i.e., sadness, anger, anxiety, or tension) but did find a positive association between PTSD symptom severity and average intensity of two negative states (i.e., anxiety and tension). Differences in methodologies could account for discrepant findings at least in part (e.g., sampling time frame of one day in that study vs. seven days in the present study). Although it is difficult to draw firm conclusions 
from only two studies, these findings taken together highlight the possibility that different dimensions or facets of negative emotional experiences may be differentially associated with PTSD.

Some have emphasized that "the separate assessment of intensity and frequency of affect is necessary to reveal such differences [in emotional experiences] between individuals" (Schimmack \& Diener, 1997.p. 1314). This distinction should be further investigated in future studies of emotion and PTSD as it has a number of implications for not only advancing and refining our understanding of emotional disruptions in PTSD but also for its treatment. For example, as some have advocated for an ideographic approach to treating the emotion regulation difficulties among patients with PTSD (Frewen \& Lanius, 2006), it seems possible that different intervention strategies might be more effective for individuals who experience negative emotions more intensely (e.g., mindfulness, relaxation, and distraction skills?) (Frewen \& Lanius, 2006) than for individuals who experience negative emotions more frequently but not more intensely on average (e.g., pleasant event scheduling?) (Diener, Sandvik, \& Pavot, 2009).

\section{Positive Emotion}

Contrary to theoretical propositions and this author's expectations, positive emotion levels were not related to PTSD symptom severity, nor did findings change when frequency and intensity of positive emotion were considered separately. Rather, results indicated that differences in positive emotion levels among women in this study were largely attributable to differences in trait negative affectivity. This finding stands in contrast to existing diary studies which, in general, have demonstrated lowered mean levels of positive emotion among individuals with PTSD compared to individuals without 
PTSD (e.g., Beckham et al., 2000; Kashdan, Julian et al., 2006). In the one diary study involving women, for example, PTSD symptom severity was negatively associated with the occurrence, but not intensity, of positive emotion (i.e., joy), even after controlling for a derived index that the authors labeled neuroticism (Newton \& Ho, 2008).

There are a number of plausible explanations that may account, in part, for the discrepancies in findings with respect to PTSD and positive emotion. First, although Newton and Ho (2008) did control for effects of neuroticism, this was an index created post-hoc from a measure of symptoms rather than a validated trait-based measure. Therefore, it seems possible that this index could be measuring a different underlying construct, or constructs, than that measured in the present study. However, because no other experience sampling studies of daily emotion and PTSD have accounted for the effects of trait negative affectivity, the few data available make conclusions in this regard difficult. Thus, while it may be that the association observed between positive emotion and PTSD in existing diary studies reflects trait differences in the tendency to experience positive emotions less frequently in general, this is speculative and requires further investigation.

Second, differences in methodologies across studies, and specifically, sampling time frame and sampling density, could also account for differences in findings. That is, the present study assessed emotional experiences roughly five times a day, on average, for seven days. In contrast, among the previous experience sampling studies that assessed positive emotion, two assessed momentary emotion over the course of a single day while concurrently sampling blood pressure (i.e., Beckam et al., 2000; Newton \& Ho, 2008). In these studies, greater sampling density over a shorter sampling time period 
(e.g., an average of 22 ratings over the course of a 13-hour period in Newton \& Ho, 2008) may be providing a slightly different picture, and perhaps a more fine-grained picture, of daily positive emotional experiences among individuals with trauma exposure, albeit emotional experiences occurring within the time frame of a single day. Thus, while the association between PTSD and negative emotion may be relatively robust and less affected by differences in study methodologies, the same may not be true for positive emotion. As many have advocated for careful consideration of sampling time frame and density of sampling which takes into account the duration and frequency of the phenomena under study (e.g., Stone \& Schiffman, 2002), this may be a particularly important issue when designing studies of naturalistic emotion in PTSD and warrants additional consideration.

Differences in sampling time frame and density among studies also speak to the utility of considering other time-related facets of emotional experiences which have not yet been investigated, such as duration, in attempts to better describe and understand emotional disruptions in PTSD, and specifically disruptions in positive emotion (Diener et al., 2009). For example, although not directly testing duration of emotional experiences, at least two diary studies have demonstrated associations between PTSD and greater variability (Newton \& Ho, 2008) or instability (Kashdan, Julian et al., 2006) of positive emotion. Drawing from these findings, it seems possible that positive emotional experiences may also be more fleeting among individuals with PTSD, or perhaps positive emotions get "interrupted" more often, reflecting a greater susceptibility to wane as other negatively appraised internal or external cues, situations, or events arise. 
Finally, although previous diary studies are generally consistent with theories proposing lower levels of positive emotion in PTSD, it is worth noting that this relationship has not been entirely supported in laboratory studies. For example, Orsillo et al. (2004) found no difference in the number of positive emotion words used to describe their responses to emotional stimuli among women with PTSD as compared to women without PTSD. Furthermore, in this study, women with PTSD actually responded with more subjectively-rated positive emotion to stimuli designed to elicit anger and fear than did women without PTSD (Osrillo et al., 2004). Likewise, Litz et al. (2000) found no differences in positive emotional responses to emotional stimuli when comparing male veterans with and without PTSD. Thus, it appears that the purported disruptions in positive emotion in PTSD are likely more complex than previously considered and have not yet been fully illuminated.

In sum, while findings with respect to negative emotion appear relatively robust in general, the relationship of positive emotion to PTSD remains less clear and may be more sensitive to other factors, including within-persons (i.e., exposure to negative events or cues) and between-persons (e.g., personality) variables, as well as study methodology. Considering laboratory and existing diary studies, including those of the present study, it appears that additional work is needed to better characterize and understand the emotional disruptions associated with PTSD, and particularly with respect to experiences of positive emotion. Given arguments that subjective well-being is best characterized by the frequent occurrence of positive affect (Diener et al., 2009), and given that improving the well-being and quality of life of individuals remains the ultimate goal of PTSD 
treatments, research to further understanding of the positive emotional experiences in everyday life of individuals with PTSD seems a worthy endeavor.

Hypotheses Set 2: Does PTSD moderate associations between stress and daily emotion?

Studies of emotional reactivity to stress have revealed negative associations

between daily stress and emotion among individuals, including those with prior trauma exposure (e.g., Glaser et al., 2006); however, no studies have directly examined daily stress in the study of PTSD and naturalistic emotion. In the present study, it was reasoned that daily stress may account for, at least in part, the observed emotional disruptions in PTSD.

Negative emotion

Results indicated that overall, daily stress levels were significantly related to higher levels of daily negative emotion and lower levels of positive emotion, as previous diary research has shown (van Eck et al., 1998; Glaser et al., 2006). That is, on days that women reported high stress levels compared to their average or typical stress level, they did indeed report greater levels of negative emotion and lower levels of positive emotion. Furthermore, in line with hypotheses, the effect of stress on negative emotion increased as a function of PTSD symptom severity such that women with more severe PTSD symptoms reported greater levels of negative emotion, compared to women with less severe PTSD symptoms, on days of greater stress. Again, this association held after accounting for trait negative affectivity, suggesting that although trait negative affectivity is associated with heightened emotional reactivity to daily stressful events among individuals in general (e.g., Mroczek \& Almeida, 2004), it did not appreciably add to the 
relationship observed between stress and negative emotion among women in the present study.

In what ways might PTSD contribute to greater increases in negative emotion in the face of stress? It seems possible that individuals with more severe PTSD symptoms may either perceive daily events as more stressful or respond with heightened reactivity to stress. There is some support for this idea. For example, a recent study found that outpatients diagnosed with PTSD provided significantly higher ratings of perceived stress (i.e.. assessed with the question "how much were you set back by stressful events, such as ...?"” p. 251 ) compared to either outpatients diagnosed with other anxiety disorders (i.e., panic disorder) or individuals in the general population (Connor, Vaishnavi, Davidson, Sheehan, \& Sheehan, 2007). Likewise, laboratory studies assessing responses to "generic", as opposed to trauma-related stressors, have demonstrated increased emotional (i.e., self-reported anxiety) and physiological (i.e., heart rate) responses to simulated work-related stressors among police officers with previous trauma exposure (Regher, LeBlanc, Jelley, Barath, \& Daciuk, 2007) as well as greater negative emotional responses (i.e., angry, fearful, anxious, disgusted, and ashamed) to mental arithmetic tasks among female survivors of childhood abuse (McDonaugh-Coyle, McHugo, Friedman, Schnurr, Zayfert, \& Descamps, 2001).

It is generally accepted that psychobiologically-mediated pathways likely underlie the physiological disturbances following trauma exposure (e.g., Pitman et al., 2006). Indeed, citing recent data from neuroimaging studies, others have proposed compromised "neural capacities for managing-regulating stress-arousal levels" in PTSD as a result of chronic trauma exposure, and particularly among those exposed to chronic interpersonal 
trauma such as domestic violence (Frewen \& Lanius, 2006, p. 111). Of course, it is unfortunately not possible to determine whether the greater negative emotion on stressful days among women with greater PTSD symptoms observed in the present study reflects individual differences in objective exposure to stressors, perceptions of stress, heightened emotional and/or physiological stress reactivity, or some interaction of these factors, all of which could reflect either post- or pre-trauma phenomena or both. Nonetheless, given that trait negative affectivity was not a significant predictor of the relationship between negative emotion and stress, it is tempting to speculate that this relationship might, at least in part, reflect a post-trauma emotional vulnerability to stress, and may also help begin to elucidate underlying causes of the "hyperemotionality" described among individuals with PTSD.

Future studies may at least begin to this tease apart by including more sophisticated and detailed assessment of stressor exposure in naturalistic studies and by the use of non trauma-related stressors that more closely approximate "real-world" stressors in laboratory studies. For example, for women exposed to interpersonal trauma such as IPV, it may be that heightened emotional reactivity occurs more readily in the face of specific kinds of stressors, such as interpersonal conflict. Interestingly enough, and lending some credibility to this proposition, some negative emotions, and specifically fear and shame, did occur more frequently on average in social settings versus non-social settings among the IPV-exposed women in the present study.

\section{Positive emotion}

In contrast, and contrary to expectation, although there was significant variation in the relationship between positive emotion and stress, PTSD did not significantly 
contribute to explaining these individual differences, nor did trait negative affectivity. As with stress and negative emotion, it seems possible that individual differences in daily stressor exposure and stress reactivity could also account for differences in the relationship between positive emotion and stress, albeit in this case independently of PTSD symptoms. However, given the characteristics of the present study sample (i.e., women in midlife with prior IPV exposure), it seems that individual differences related to coping and resiliency may also help explain differences in these associations. For example, evidence suggests increasing complexity in the emotional experiences of individuals as they age, including the more frequent co-occurrence of negative and positive emotions (e.g., Ong \& Bergeman, 2004). Specifically, in one study involving older individuals, neuroticism and perceived stress were associated with less cooccurrence of positive and negative emotion during stressful events; however, "dispositional resilience" was associated with greater co-occurrence of positive and negative emotion during stressful events (Ong \& Bergeman, 2004; p. 117).

Folkman (2008) argues that while positive emotions have received little attention in the stress and coping literature compared to negative emotions, they have important functions in the stress response and are related to coping processes, such as finding meaning in stressful events, which are different from those which regulate distress. In a similar vein, a study of posttraumatic growth among women with previous experiences of IPV found that, although one dimension of growth, appreciation for life, was significantly associated with prior IPV experiences, it was unrelated to current level of psychological distress, or depression (Cobb, Tedeschi, Calhoun, \& Cann, 2006). 
When taken together, these studies suggest that the negative effects of daily stress on positive emotion among the IPV-exposed women of the present study may have been buffered through the use of specific coping strategies, such as drawing on a greater appreciation for life or finding meaning in negative or stressful daily events. That is, either meaning-based coping or resiliency to stress may allow for the continued ability to experience positive emotion, or perhaps experience less of a reduction in positive emotion, during stress or negative events. Furthermore, as Folkman (2008), Ong \& Bergeman (2004), and Tedeschi et al (2006) suggest, this may occur independently of, or perhaps with the co-occurrence of, negative emotion or other indicators of psychological distress. Although appearing as a plausible explanation, with no studies with which to directly compare findings, this at best remains at best a hypothesis in need of additional examination.

In sum, although differences in the relationship between positive emotion and stress remains unexplained, at least in terms of the factors measured in the present study, differences in coping, resiliency, and trauma-related growth hold promise for furthering understanding of this relationship among trauma-exposed individuals. Furthermore, assessment of these factors in future studies may ultimately prove fruitful in clarifying discrepant findings among studies of PTSD and positive emotion more generally.

Hypothesis Set 3: Are daily PTSD symptoms associated with daily emotion levels?

Another major aim of the present study was to empirically test theoretical propositions of emotional disruptions in PTSD using naturalistic data. Specifically, the relationship between daily reexperiencing symptoms and negative emotional experiences, as well as the temporal relationship between daily reexperiencing and emotional numbing 
symptoms, were tested. In this regard, it was expected that higher levels of daily reexperiencing symptoms reported at the end of the day would be associated with higher levels of mean daily negative emotion reported throughout the day. This hypothesis was not supported, however, as daily reexperiencing symptoms were not significantly associated with overall daily negative emotion levels.

While at odds with theoretical formulations linking experiences of negative emotion to the reexperiencing of traumatic events (Foa et al., 1998; Horowitz, 1986; Litz, 1992), it is worth noting that this finding is not entirely inconsistent with laboratory studies. That is, while one study did find greater negative emotional responses to traumarelated stimuli among veterans with PTSD (i.e., Pitman et al., 1987), in other studies, exposure to trauma cues (i.e., trauma priming) did not increase negative emotional responses to subsequent non trauma-related stimuli among individuals with PTSD (e.g., Litz et al., 2000; Wagner et al., 2003). Thus, the limited existing data from laboratory studies along with the naturalistic data from the present study do not appear to support theoretical formulations positing increased negative emotional experiences in PTSD as driven by traumatic reexperiencing, at least at first glance.

However, before casting doubt on such theories, it also seems possible that traumatic reexperiencing perhaps does not affect emotional experiences by increasing negative emotion levels overall as was hypothesized, but rather, results in negative emotional experiences that are more variable (Kashdan, Julian et al., 2006; Newton \& Ho, 2008). In other words, perhaps negative emotion levels "rise" and "fall" throughout the day as intrusive reexperiencing symptoms are experienced and then fade. This speaks to a relationship best captured by examination of emotional experiences which unfold 
over time, and as such, it could be expected that existing laboratory studies were not designed to capture such a relationship or process. Although not assessing daily reexperiencing symptoms, Newton and Ho (2008) provide some support for this notion in that they reported a positive association between past-month reexperiencing symptoms and intraindividual variability of momentary experiences of anxiety. That is, while women with more severe reexperiencing symptoms over the prior month did not experience greater levels of anxiety overall, they did experience more "ups and downs" of anxiety during the assessment period.

As the present study is the only the first to explicitly test such formulations empirically through naturalistic assessment of not only emotions but also PTSD symptoms, replication is certainly needed before forming any firm conclusions. However, if indeed traumatic reexperiencing manifests as more variable negative emotional experiences rather than a general increase in overall levels, as in laboratory studies, it seems possible that the relationship between negative emotion and reexperiencing symptoms may simply have not been captured in the present study. Thus, although not supportive of theoretical propositions, this finding is nonetheless important in that it offers a point of comparison for expanded (i.e., inclusion of variability of negative emotions) and more rigorous testing of such theoretical propositions in future studies.

\section{Emotional Numbing}

With respect to temporal relationships among daily symptoms, it was expected that emotional numbing symptoms on a given day would be greatest among women who, on the previous day, experienced high levels of reexperiencing symptoms and also high levels of negative emotion. Although a significant interaction between previous day 
reexperiencing symptoms and negative emotion emerged as hypothesized, the nature of the relationship differed from what was expected. That is, the effect of high levels of reexperiencing symptoms on subsequent emotional numbing symptoms was dependent on the experience of low, rather than high, levels of negative emotion on preceding days. More specifically, among women who reported high levels of reexperiencing symptoms, those who reported high levels of negative emotion evidenced the lowest levels of emotional numbing on subsequent days, while those who reported low levels of negative emotion evidenced the highest level of emotional numbing on subsequent days.

At their simplest, these findings provide some support for temporal linkages between reexperiencing and emotional numbing symptoms. However, while these findings point to a phasic relationship between these specific symptoms, at least in part, the association does not appear to be driven by the experience of intense negative emotion as some theories propose (e.g., Horowitz, 1986). Rather, drawing from the present study`s findings, it is proposed that there are different response processes which occur with respect to traumatic reexperiencing and which influence the presence of subsequent emotional numbing. More specifically, one process appears to be characterized by responding to intrusions with relatively low levels of negative emotion and results in high levels of emotional numbing. In line with conceptualizations of emotional numbing as a phasic response to traumatic reminders and described as analogous to "stress-induced analgesia" observed among animals exposed to unpredictable stressors (e.g., Foa, Zinbarg, \& Rothbaum, 1992), this seemingly fits with the descriptions of "hypoemotionality" observed among individuals with PTSD (Litz \& Gray, 2002). Another process appears to be characterized by responding to intrusions 
with high levels of negative emotions, resulting in relatively low levels emotional numbing symptoms and which fits with descriptions of "hyperemotionality" described among individuals with PTSD (Litz \& Gray, 2002). Notably, these two processes also mirror classes of peritraumatic emotional responses to traumatic events more broadly (i.e., dissociative-type responses vs. the experience of intense negative emotions, such as fear, anger, and shame), both of which have been implicated in the development of PTSD (Ozer, Best, Lipsey, \& Weiss, 2003; Andrews, Brewin, Rose, \& Kirk, 2000). See Figure 3.

The proposal of differentiated responses to traumatic reexperiencing has a number of implications for advancing understanding of emotional processes in PTSD. First, as mentioned, such an explanation can account for the seemingly paradoxical occurrence of both "hypoemotionality" and "hyperemotionality" observed among individuals with PTSD symptoms, which some theories have been unable to adequately explain (e.g., Litz et al., 2000). At the same time, it is worth pointing out, however, that associations were examined within-day and across women in the present study, and as such, it cannot be determined whether these seemingly distinct response processes occurred in any given individual on different days, or alternatively, represent more stable, characteristic responses to intrusions. Given that a substantial amount of variance in emotional numbing symptoms was attributable to between-subjects factors (i.e., nearly $70 \%$ ), which were not included in the model, at least in the present sample, this seems possible and is a prospect that could be explored in future studies.

Second, such an explanation provides a potential framework for continued study of emotional processes in PTSD and could provide clues for how we may begin to more 
accurately operationalize and assess constructs associated with emotional disruptions in PTSD. For example, many have begun to criticize DSM-IV emotional numbing criteria because they do not provide information about the actual process or parameters of emotional functioning in PTSD and have called for better operationalization of emotional numbing phenomena more generally (Litz \& Gray, 2002; Orsillo, Theodore-Oklota, Luterek, \& Plumb, 2007). Although the present study relied on DSM-IV based criteria for measuring emotional numbing symptoms, individuals who reported high levels of emotional numbing symptoms in their day-to-day lives did indeed report diminished emotional experiences, albeit negative emotional experiences, presumably in response to stimuli capable of producing high levels of negative emotion. It is argued that longitudinal, naturalistic studies of daily emotions and symptoms provide a unique opportunity to begin to uncover the nature of emotional numbing phenomena and should be utilized. Also, given concern over current diagnostic criteria, future studies would likely benefit from more sophisticated assessment of emotional numbing phenomena via examination of actual emotional experiences rather than relying on diagnostic criteria.

Finally, the idea of differentiated responses to traumatic reexperiencing has treatment implications and may aid in identifying individuals who are most likely to benefit from particular PTSD treatments. For example, contemporary exposure-based treatments for PTSD rely on the activation of intense emotion upon controlled exposure to trauma memories for the resolution of PTSD symptoms (Foa \& Rothbaum, 1998), and evidence suggests that the presence of emotional numbing is associated with poorer treatment outcome (Foa, Riggs, Massie, \& Yarczower, 1995) and psychosocial functioning in general (Kuhn, Blanchard, \& Hickling, 2003). Thus, women who 
experienced high levels of reexperiencing but low levels of negative emotion may be those who fare worse in exposure treatments. It seems possible that these are individuals who may benefit from emotional regulation skills training before engaging in exposurebased treatments, as some have suggested (Cloitre et al., 2002). On the other hand, women who experienced high levels of negative emotion in response to reexperiencing symptoms may be the very individuals who benefit directly from exposure treatments. The reduction of reexperiencing symptoms may be adequate to resolve the emotional disruptions these individuals experience, as others have suggested (Litz, 1992; Litz et al., 2000; Litz \& Gray 2002).

\section{Limitations, Special Considerations, and Future Directions}

Although making a number of unique contributions and improvements to the existing literature on PTSD and emotion, the findings of the present study must be placed within the context of some limitations and other special considerations. First, given that the present sample represents a very small segment of the population of trauma-exposed individuals (i.e., women in midlife with prior IPV exposure), the degree that these findings may be generalized to other trauma-exposed groups (e.g., combat veterans, men and women exposed to non-interpersonal traumas such natural disasters) is limited. Furthermore, it is not clear to what degree the present sample is representative of other populations of IPV-exposed women. That is, in addition to differences which may arise from the age restrictions placed on the sample, women appeared to be well-educated (i.e., over half held a college degree or had professional training), relatively high functioning (i.e., over half were employed), and in general, had relatively frequent experiences of positive emotion in their daily lives. Although descriptive data suggested that, on 
average, women were exposed to IPV that could be characterized as chronic and severe and reported levels of PTSD symptoms which were moderate in severity, it may be that this particular sample of women represents a particularly resilient group of women or women who possess other resources which have otherwise allowed them to maintain a relatively high level of functioning.

Second, the present study measured momentary experiences of emotion which were assessed randomly within specified periods of time throughout the day. Thus, the present study provided a random sample of women`s emotional experiences as they occurred across the course of a typical week. As such, the present study reveals information about women's emotional experiences rather than emotional responses, such as those which may be measured in more controlled laboratory settings but which lack ecological validity. This is an important distinction that deserves additional consideration in future naturalistic studies. For example, although the present study did measure global stress through one end-of-the day assessment item, emotions in response to specific stressors or events were not directly measured. Assessment of emotional responses to specific events in daily life could begin to uncover important information about the processes underlying emotional disruptions in PTSD, particularly given associations between PTSD, negative emotion, and stress found in the present study.

On a related note, the study was entirely self-report, and as such, only one channel of emotion was considered, leaving other, potentially important aspects of emotional experiences unexplored. Laboratory studies examining multiple channels of emotion, for example, have found differing findings with respect to expression of emotion assessed via facial EMG as opposed to self-reported "felt" emotion (e.g., Litz et al., 2000). 
Studying how channels of emotional experiences may differentially be affected by PTSD symptomatology is important for a number of reasons. First, clinical interventions target not only subjective "felt" emotion but also the physiological aspects of emotion (e.g., relaxation training to target physiological components of fear or anxiety) as well as expression (e.g., social skills training often assists individuals in effectively communicating emotions such as anger or sadness). Second, the study of multiple channels of emotion, and particularly physiological facets of naturalistic emotional experiences, may also begin to uncover mechanisms contributing to negative health outcomes and poorer physical health observed among individuals with PTSD (Schnurr \& Green, 2004).

Finally, a strength of the present study was the selection of a specific emotion theory framework (i.e., a discrete emotions theoretical framework) from which emotion assessment measures and study rationale were derived. Although examining the relationship between PTSD and specific emotions was beyond the scope of this paper, future research could and should incorporate this as specific emotions may differentially act to influence different aspects of women's health and psychosocial functioning. For instance, there is some evidence to suggest that seemingly similar discrete emotions differently affect immune-related responses. In one study, for example, women who wrote about past traumatic experiences experienced both increases in self-reported guilt and shame as compare to women in a neutral writing condition; however, only shame, and not guilt, was associated with indicators of proinflammatory cytokine activity among women who wrote about past trauma (Dickerson, Kemeny, Aziz, Kim, \& Fahey, 2004). Likewise, anger has been shown to interfere with the efficacy of exposure treatments for 
PTSD (Foa et al., 1995). Thus, elucidating relationships between PTSD, emotion, and health more broadly could be greatly aided by naturalistic studies, and approaching such lines of research with a well-specified theoretical framework of emotion would be beneficial.

\section{Clinical Applications}

Overall, while the present study highlights the value of using experience sampling methodologies to the study of PTSD, it also points to the value of applying these methodologies, and particularly the use of electronic diaries, to clinical interventions aimed at treating PTSD. First, having a more complete understanding of individuals' day-to-day emotional experiences could be an invaluable aid to the treating clinician. That is, discussed previously, the experience of specific emotions such as anger as well as the presence of emotional numbing have been shown to interfere with otherwise effective, empirically supported treatments for PTSD (e.g., Foa et al., 1995). These emotional disruptions, which may or may not be readily observable to the clinician, manifest in clinical settings, or be directly reported by patients, may be revealed through such methodologies as used in the present study. On a related note, given that the aim of self monitoring is to increase awareness of one's behavior, daily assessment of emotional experiences may also aid the individual by increasing self-awareness of the exact nature of their emotional difficulties. Given that emotion regulation difficulties are a driving force in motivating individuals to seek treatment (Cloitre et al., 2002), tailoring treatment to a particular individual's difficulties may render treatment more effective (Frewen \& Lanius, 2006). 


\section{Summary and Conclusions}

The present study contributed to the relatively small but growing body of empirical evidence attempting to shed light on emotional experiences and PTSD by assessing discrete, momentary emotional experiences, stress, and symptoms of PTSD among women with prior experiences of IPV. Major findings from the present study included the following:

1) Past-month PTSD symptomatology was associated with greater levels of negative emotion in daily life. This association appears to be a relatively robust finding as it has generally been supported across both laboratory and naturalistic studies. Additionally, emerging evidence from the present study and that of Newton and Ho (2008) suggests that different facets of negative emotional experiences may be differentially associated with PTSD symptomatology. Finally, the moderating effect of past-month PTSD symptom severity on associations between daily stress and daily negative emotion found in the present study suggest that the relationship between PTSD and negative emotion may partially result from increased emotional responding to daily stressful events.

2) Past-month PTSD symptomatology was not associated with levels of positive emotion in daily life, and PTSD did not contribute to observed differences in the relationship between daily stress levels and positive emotion. Thus, the relationship between PTSD and positive emotion remains unclear, although it is suspected that other between-persons factors, such as resiliency and coping, likely play a role in buffering the effects of stress, including both traumatic stress symptoms as well as daily stressors. 
3) Daily intrusive symptoms were not associated with heightened overall levels of negative emotions; however, it seems likely that this relationship may be better captured by greater variability in negative emotional responses rather than overall higher levels of negative emotion. Likewise, lagged analyses revealed that high levels of emotional numbing symptoms were predicted by the interaction of prior day reexperiencing symptoms and low levels, rather than high levels, of negative emotions. From this, it is proposed that emotional numbing results from differentiated responses to trauma cues which may be similar to peritraumatic emotional responses of dissociation.

In sum, although few have been conducted to date in the area of PTSD and emotion, experience sampling studies offer the opportunity to study the emotional experiences as they unfold in course of everyday among individuals affected by traumatic experiences. As such, they have utility in both research and clinical settings. The present study used such methodologies, as well as electronic diaries, to study naturally-occurring experiences of emotion, stress, and symptoms of PTSD among women with prior IPVexposure and made a number of important of contributions to the existing literature, particularly with respect to examining temporal relationships between symptoms and emotions. Future studies seeking to advance understanding of emotional disruptions in PTSD should consider utilizing such methodologies and may wish to use the present study as a guide for developing more refined hypotheses and sophisticated studies. 


\section{REFERENCES}

Affleck, G., Zatura, A., Tennen, H., \& Armeli, S. (1999). Multilevel daily process designs for consulting and clinical psychology: A preface for the perplexed. Journal of Consulting and Clinical Psychology, 67(5), 746-754.

Aiken, L. S., \& West, S. G. (1991). Multiple regression: Testing and interpreting interactions. Newbury Park, CA: Sage.

Amaya-Jackson, L., Davidson, J. R., Hughes, D. C., Swartz, M., Reynolds, V., George, L. K., et al. (1999). Functional impairment and utilization of services associated with posttraumatic stress in the community. Journal of Traumatic Stress, 12(4), 709-724.

Amdur, R. L., Larsen, R., \& Liberzon, I. (2000). Emotional processing in combat-related posttraumatic stress disorder: A comparison with traumatized and normal controls. Journal of Anxiety Disorders, 14(3), 219-238.

American Psychiatric Association. (1994). Diagnostic and statistical manual of mental disorders ( $4^{\text {th }}$ ed.). Washington, DC: Author.

Andrews, B., Brewin, C. R., Rose, S., \& Kirk, M. (2000). Predicting PTSD symptoms in victims of violent crime: The role of shame, anger, and childhood abuse. Journal of Abnormal Psychology, 109(1), 69-73. 
Basile, K. C., Arias, I., Desai, S., \& Thompson, M. P. (2004). The differential association of intimate partner physical, sexual, psychological, and stalking violence and posttraumatic stress symptoms in a nationally representative sample of women. Journal of Traumatic Stress, 17(5), 413-421.

Barrett, D. J, \& Feldman Barrett, L. F. (2000). The Experience Sampling Program (ESP). Available: (http://www2.bc.edu/ barrettli/esp/)

Baumeister, R. F., Stillwell, A. M., \& Heatherton, T. F. (1994). Guilt: An interpersonal approach. Psychological Bulletin, 115(2), 243-267.

Beckham, J. C., Feldman, M. E., Barefoot, J. C., Fairbank, J. A., Helms, M. J., Haney, T. L. et al. (2000). Ambulatory cardiovascular activity in Vietnam combat veterans with and without posttraumatic stress disorder. Journal of Consulting and Clinical Psychology, 68(2), 269-276.

Blank, A. (1993). The longitudinal course of posttraumatic stress disorder. In J. Davidson \& E. Foa (Eds.), Posttraumatic stress disorder: DSM-IV and beyond. Washington, DC: American Psychiatric Press.

Bleuler, E. (1924). Texthook of psychiatry. (A. A. Brill, Trans.). New York: The Macmillan Company.

Bolger, N., Davis, A., \& Rafaeli, E. (2003) Dairy methods: Capturing life as it is lived. Annual Review of Psychology, 54,579-616.

Bolton, E. E., Gray, M. J., \& Litz, B. T. (2006). A cross-lagged analysis of the relationship between symptoms of PTSD and retrospective reports of exposure. Anxiety Disorders, 20, 877-895. 
Booth, R. J., \& Pennebaker, J. W. (2000). Emotions and immunity. In M. Lewis and J. M. Haviland-Jones (Eds.), Handbook of Emotions ( $2^{\text {nd }}$ ed., pp. 558-570). New York: Guildford.

Brantley, P. J., Waggoner, C. D., Jones, G. N., \& Rappaport, N. B. (1987). Journal of Behavioral Medicine, 10(1), 61-74.

Bunce, S. C., Larsen, R. J., \& Peterson, C. (1995). Life after trauma: Personality and daily life experiences of traumatized people. Journal of Personality, 63(2), $165-$ 188.

Burton, C., Weller, D., \& Sharpe, M. (2007). Are electronic diaries useful for symptoms research? A systematic review. Journal of Psychosomatic Research, 62,553-561.

Carstensen, L. L., \& Charles, S. T. (1998). Emotion in the second half of life. Current Directions in Psychological Science, 7(5), 144-149.

Carstensen, L. L., Pasupathi, M., Mayr, U., \& Nesselroade, J. R. (2000). Emotional experience everyday across the adult lifespan. Journal of Personality and Social Psychology, 79(4), 644-655.

Cerin, E., \& Barnett, A. (2006). A processual analysis of basic emotions and sources of concerns as they are lived before and after a competition. Psychology of Sport and Exercise, 7(3), 287-307.

Charles, S. T. (2005). Viewing injustice: Greater emotion heterogeneity with age. Psychology and Aging, 20, 159-164.

Chemtob, C. M., Novaco, R. W., Hamada, R. S., Gross, D. M., \& Smith, G. (1997) Anger regulation deficits in combat-related posttraumatic stress disorder. Journal of Traumatic Stress, 10(1), 17-36. 
Christensen, T. C., Feldman Barrett, L., Bliss-Moreau, E., Lebo, K., \& Kaschub, C. (2003). A practical guide to experience-sampling procedures. Journal of Happiness Studies, 4, 53-78.

Cicchetti, D., Ackerman, B. P., \& Izard, C. E. (1995). Emotions and emotion regulation in developmental psychopathology. Development and Psychopathology, 7, 1-10.

Cloitre, M., Koenen, K. C., Cohen, L. R., \& Han, H. (2002). Skills training in affective and interpersonal regulation followed by exposure: A phase-based treatment for PTSD related to childhood abuse. Journal of Consulting and Clinical Psychology, 70(5), 1067-1074.

Cloitre, M., Miranda, R., Stovall-McClough, K., \& Han., H. (2005). Beyond PTSD: Emotion regulation and interpersonal problems as predictors of functional impairment in survivors of childhood abuse. Behavior Therapy, 36(2), 119-124.

Cobb, A. R., Tedeschi, R. G., Calhoun, L. G., \& Cann, A. (2006). Correlates of posttraumatic growth in survivors of intimate partner violence. Journal of Traumatic Stress, 19(6), 895-903.

Cohen, J. (1988). Statistical power analysis for the behavioral sciences $\left(2^{\text {nd }} \mathrm{ed}\right.$.). Hillsdale, NJ: Lawrence Earlbaum Associates.

Collins, R. L., Ellickson, P., Hays, R., McCaffrey, D. F. (2000). Effects of incentive size and timing of response rates to a follow-up wave of a longitudinal mailed survey. Evaluation Review, 24, 347-363.

Connor, K. M., Vaishnavi, S., Davidson, J. R. T., Sheehan, D. D., \& Sheehan, K. H. (2007). Perceived stress in anxiety disorders and the general population: A study of the Sheehan stress vulnerability scale. Psychiatry Research, 151, 249-254. 
Cosmides, L., \& Tooby, J. (2000). Evolutionary Psychology and the emotions. In M. Lewis and J. Haviland-Jones (Eds.), Handbook of emotions ( $2^{\text {nd }}$ ed., pp. 91-115). New York: Guilford.

Costa, P. T., \& McCrae, R. R. (1992). Normal personality assessment in clinical practice: The NEO Personality Inventory. Journal of Personality Assessment, 4, $5-13$.

Cox, B. J., MacPherson, P. S. R., Enns, M. W., McWilliams, L. A. (2004). Neuroticism and self-criticism associated with posttraumatic stress disorder in a nationally representative sample. Behaviour Research and Therapy, 42, 105-114.

Dickerson. S. S., Kemeny, M. E., Aziz, N., Kim, K. H., \& Fahey, J. L. (2004). Immunological effects of induced shame and guilt. Psychosomatic Medicine, 66, 124-131.

Diehl, M., Coyle, N., \& Labouvie-Vief, G. (1996). Age and sex differences in coping and defense across the life-span. Psychology and Aging, 11, 127-139.

Diener, E., Larsen, R. J., Levine, S., \& Emmons, R. A. (1985). Intensity and frequency: The underlying dimensions of positive and negative affect. Journal of Personality and Social Psychology, 48, 1253-1265.

Diener, E., \& Lucas, R. E. (2000). Subjective emotional well-being. In M. Lewis and J. M. Haviland-Jones (Eds.), Handhook of emotions ( $2^{\text {nd }}$ ed., pp. 325-337). New York: Guildford.

Diener, E., Sandvik, E., \& Pavot, W. (2009). Happiness is the frequency, not the intensity of positive versus negative affect. In E. Diener (Ed.), Assessing well- 
heing: The collected works of Ed Diener (pp. 213-231). New York: Springer

Business Media.

Eaton, L. G., \& Funder, D. C. (2001). Emotional experience in daily life: Valence, variability, and rate of change. Emotion, 1(4), 413-421.

Ehlers, A., \& Clark, D. M. (2000). A cognitive model of posttraumatic stress disorder. Behaviour Research and Therapy, 38, 319-345.

Eid, M., \& Diener, E. (1999). Intraindividual variability in affect: Reliability, validity, and personality correlates. Journal of Personality and Social Psychology, 76(4), $662-676$.

Ekman, P. (1992). An argument for basic emotions. Cognition and Emotion, 6, 169-200.

Elhai, J. D., Engdahl, R. M., Palmieri, P. A., Naifeh, J. A., Schweinle, A., \& Jacobs, G. A. (2009). Assessing posttraumatic stress disorder with or without reference to a single, worst traumatic event: Examining differences in factor structure. Psychological Assessment, 21(4), 629-634.

Feiring, C., Taska, L., \& Chen, K. (2002). Trying to understand why horrible things happen: Attributions, shame, and symptom development following sexual abuse. Child Maltreatment, 7(1), 26-41.

Feldman Barrett, L., \& Barrett, D. J. (2001). An introduction to computerized experience sampling in psychology. Social Science Computer Review, 19(2), 175-185.

Flack, W. F., Jr., Litz, B. T., Hsieh, F. Y., Kaloupek, D. G., \& Keane, T. (2000). Predictors of emotional numbing, revisited: A replication and extension. Journal of Traumatic Stress, 13(4), 611-618. 
Foa, E. B., Riggs, D. S., \& Gershuny, B. S. (1995). Arousal, numbing, and intrusion: Symptom structure of PTSD following assault. The American Journal of Psychiatry, 152(1), 116-120.

Foa, E. B., Riggs, D. S., Massie, E. D., Yarczower, M. (1995). The impact of fear activation and anger activation on the efficacy of exposure treatment for posttraumatic stress disorder. Behavior Therapy, 26, 487-499.

Foa, E. B., \& Rothbaum, B. O. (1998). Treating the trauma of rape: Cognitivebehavioral therapy for PTSD. New York: Guilford.

Foa, E. B., Steketee, G., \& Rothbaum, B. O. (1989). Behavioral/cognitive conceptualization of post-traumatic stress disorder. Behavior Therapy, 20(2), 155-176.

Foa, E., Zinbarg, R., \& Rothbaum, B. (1992). Uncontrollability and unpredictability in posttraumatic stress disorder: An animal model. Psychological Bulletin, 112, 218-238.

Folkman, S. (2006). The case for positive emotions in the stress process. Anxiety, Stress, \& Coping, 2l(1), 3-24.

Frewen, P. A., \& Lanius, R. A. (2006). Toward a psychobiology of posttraumatic selfdysregulation: Reexperiencing, hyperarousal, dissociation, and emotional numbing. Annals of the New York Academy of Sciences, 1071, 110-124.

Fridja, N. H. (1986). The emotions. Cambridge, UK: Cambridge University Press.

Glaser, J., van Os, J., Portegijs, P. J. M., \& Myin-Germeys, I. (2006). Childhood trauma and emotional reactivity to daily life stressors in adult frequent attenders of general practitioners. Journal of Psychosomatic Research, 61,229-236. 
Golding, J. M. (1999). Intimate partner violence as a risk factor for mental disorders. Journal of Family Violence, 14(2), 99-132.

Gross, J. J. (1998). The emerging field of emotion regulation: An integrative review. Review of General Psychology, 2, 271-299.

Gross, J. J. (1999). Emotion regulation: Past, present, future. Cognition and Emotion, $13(5), 551-573$.

Henning, K. R., \& Frueh, B. C. (1997). Combat guilt and its relationship to PTSD symptoms. Journal of Clinical Psychology, 53(8), 801-808.

Horowitz, M. J. (1986). Stress-response syndromes ( $2^{\text {nd }}$ ed.). New York: Aronson. Izard, C. E. (1977). Human Emotions. New York: Plenum Press.

Izard, C. E., \& Ackerman, B. P. (2000). Motivational, organization, and regulatory functions of discrete emotions. In M. Lewis \& J. M. Haviland-Jones (Eds.), Handbook of emotions (2nd ed., pp. 253-264). New York: Guilford.

Izard, C. E., Libero, D. Z., Putnam, P., \& Haynes, O. M. (1993). Stability of emotion experiences and their relations to traits of personality. Journal of Personality and Social Psychology, 64(5), 847-860.

John, O. P., Donahue, E. M., \& Kentle, R. L. (1991). The Big Five Inventory--Versions $\underline{4 a}$ and 54. Berkeley, CA: University of California,Berkeley, Institute of Personality and Social Research.

Johnson, D. R., Lubin, H., Rosenheck, R., Fontana, A., Suthwick, S., \& Charney, D. (1997). The impact of the homecoming reception on the development of posttraumatic stress disorder: The West Haven Homecoming Stress Scale (WHHSS). Journal of Traumatic Stress, I0(2), 259-277. 
Johnson, D. R., Westermeyer, J., Kattar, K., \& Thuras, P. (2002). Daily charting of posttraumatic stress symptoms. Journal of Nervous and Mental Disease, 190(10), $683-692$.

Kashdan, T. B., Elhai, J. D., \& Frueh, B. C. (2006). Anhedonia and emotional numbing in combat veterans with PTSD. Behaviour Research and Therapy, 44, 457-467.

Kashdan, T. B., Julian, T., Merritt, K., \& Uswatte, G. (2006). Social anxiety and posttraumatic stress disorder in combat veterans: Relations to well-being and character strengths. Behaviour Research and Therapy, 44, 561-583.

Kashden, T. B., Uswatte, G., Steger, M. F., \& Julian, T. (2006). Fragile self-esteem and affective instability in posttraumatic stress disorder. Behaviour Research and Therapy, 44, 1609-1619.

King, D. W., King, L. A., Erickson, D. J., Huang, M. T., Sharkansky, E. J., \& Wolfe, J. (2000). Posttraumatic stress disorder and retrospectively reported stressor exposure: A longitudinal prediction model. Journal of Abnormal Psychology, $109(4), 624-633$.

King, L. A., King, D. W., Salgado, D. M., \& Shalev, A. Y. (2003). Contemporary longitudinal methods for the study of trauma and posttraumatic stress disorder. CNS Spectrums, 8(9), 686-692.

Krause, E. D., Kaltman, S., Goodman, L. A, \& Dutton, M. A. (2007). Longitudinal factor structure of posttraumatic stress symptoms related to intimate partner violence. Psychological Assessment, 19(2), 165-175. 
Krause, E. D., Kaltman, S., Goodman, L., \& Dutton, M. A. (2006). Role of distinct PTSD symptom clusters in intimate partner reabuse: A prospective study. Journal of Traumatic Stress, 19(4), 507-516.

Kring, A. M., \& Werner, K. H. (2004). Emotion regulation and psychopathology. In P. Philippot \& R. S. Feldman (Eds.), The Regulation of emotion (pp.359-385). Mahwah, NJ: Earlbaum.

Kubany, E. S., Aubeg, F. R., Owens, J. A., Brennan, J. M., Kaplan, A. S., \& Watson, S. B. (1995). Initial examination of a multidimensional model of trauma-related guilt: Applications to combat veterans and battered women. Journal of Psychopathology and Behavioral Assessment, 17(4), 353-376.

Kubany, E. S., Haynes, S. N., Aubeg, F. R., Manke, F. P., Brennan, J. M., \& Stahura, C. (1996). Development and validation of the Trauma-Related Guilt Inventory (TRGI). Psychological Assessment, 8(4), 428-444.

Kuhn, E., Blanchard, E. B., \& Hickling, E. J. (2003). Posttraumatic stress disorder and psychosocial functioning within two samples of MVA survivors. Behaviour Research and Therapy, 41, 1105-1112.

Lang, A. J., Laffaye, C., Satz, L. E., Dresselhaus, T. R., Stein, M. B. (2003). Sensitivity and specificity of the PTSD Checklist in detecting PTSD in female veterans in primary care. Journal of Traumatic Stress, 16(3), 257-264.

Lazarus, R. S. (1991). Progress on a cognitive-motivational-relational theory of emotion. American Psychologist, 46(8), 819-834.

Leskela, J., Dieperink, M., \& Thuras, P. (2002). Shame and posttraumatic stress disorder. Journal of Traumatic Stress, 15(3), 223-226. 
Leventhal, H., \& Patrick-Miller, L. (2000). Emotions and physical illness: Causes and indicators of vulnerability. In M. Lewis \& J. M. Haviland-Jones (Eds.), Handhook of emotions (2nd ed., pp.523-537). New York: Guilford.

Litz, B. T. (1992). Emotional numbing in combat-related posttraumatic stress disorder: A critical review and reformulation. Clinical Psychology Review, 12, 417-432.

Litz, B. T., \& Gray, M. J. (2002). Emotional numbing in posttraumatic stress disorder: Current and future research directions. Australian and New Zealand Journal of Psychiatry, 36,198-204.

Litz, B. T., Orsillo, S. M., Kaloupek, D., \& Weathers, F. (2000). Emotional processing in posttraumatic stress disorder. Journal of Abnormal Psychology, 109(1), 26-39.

Litz, B. T., Schlenger, W. E., Weathers, F. W., Cadell, J. M., Fairbank, J. A., \& LaVange, L. M. (1997). Predictors of emotional numbing in posttraumatic stress disorder. Journal of Traumatic Stress, 10(4), 607-616.

Liu, X., Spybrook, J., Congdon, R., Martinez, A., \& Raudenbush, S. (2006). Optimal design for multi-level and longitudinal research (Version 1.77) [Computer software]. Retrieved March 12, 2007, from http://sitemaker.umich.edu/groupbased/optimal_design_software

Marco, C. A., \& Suls, J. (1993). Daily stress and the trajectory of mood: Spillover, response assimilation, contrast, and chronic negative affectivity. Journal of Personality and Social Psychology, 64(6), 1053-1063.

Marshall, G. N., Schell, T. L., Glynn, S. M., \& Shetty, V. (2006). The role of hyperarousal in the manifestation of posttraumatic distress following injury. Journal of Abnormal Psychology, 115(3), 624-628. 
McDonagh-Coyle, A., McHugo, G. J., Friedman, M. J., Schnurr, P. P., Zayfert, C., \& Descamps, M. (2001). Psychophysiological reactivity in female sexual abuse survivors. Journal of Traumatic Stress, 14(4), 667-683.

Monson, C. M., Price, J. L., Rodriguez, B. F., Ripley, M. P., \& Warner, R. A. (2004). Emotional deficits in military-related PTSD: An investigation of content and process disturbances. Journal of Traumatic Stress, 17(3), 275-279.

Mroczek, D. K., \& Almeida, D. M. (2004). The effect of daily stress, personality, and age on daily negative affect. Journal of Personality, 72(2), 355-378.

Muraoka, M. Y., Carlson, J. G., \& Chemtob, C. M. (1998). Twenty-four hour ambulatory blood pressure monitoring in combat-related posttraumatic stress disorder. Journal of Traumatic Stress, 11(3), 473-484.

Myin-Germeys, I., Krabbendam, L., Delespaul, P., \& van Os, J. (2004). Sex differences in emotional reactivity to daily life stress in psychosis. Journal of Clinical Psychiatry, 65(6), 805-809.

Newton, T. L., \& Ho, I. K. (2008). Posttraumatic stress symptoms and emotion experience in women: Emotion occurrence, intensity, and variability in the natural environment. Journal of Psychological Trauma, 7(4), 276-297.

Nezlek, J. B. (2001). Mutlilevel random coefficient analyses of event- and signalcontingent data in social and personality psychology research. Personality and Social Psychology Bulletin, 27(7), 771-785.

Nezlak, J. B. (2003). Using multilevel random coefficient modeling to analyze social interaction diary data. Journal of Social and Personal Relationships, 20(4), 437469. 
Nicassio, P. M., Moxham, E. G., Schuman, \& Gevirtz, R. N. (2002). The contribution of pain, reported sleep quality, and depressive symptoms to fatigue in fibromyalgia. Pain, 100, 271-279.

Norrris, F. H. \& Hamblen, J. L. (2004). Standardized self-report measures of civilian trauma and PTSD. In J. P. Wilson \& T. M. Keane (Eds.), Assessing psychological trauma and PTSD (pp. 63-102). New York: Guilford.

Oatley, K. \& Johnson-Laird, P. N. (1987). Towards a cognitive theory of emotions. Cognition \& Emotion, 1, 29-50.

Ong, A. D., \& Bergemann, C. S. (2004). The complexity of emotions in later life. Journal of Geronotology: Psychological Sciences, 59B, P117-P122.

Orsillo, S. M., Batten, S. V., Plumb, J. C., Luterek, J. A., \& Roessner, B. M. (2004). An experimental study of emotional responding in women with posttraumatic stress disorder related to interpersonal violence. Journal of Traumatic Stress, 17(3), 241-248.

Orth, U., \& Wieland, E. (2006). Anger, hostility, and posttraumatic stress disorder in trauma-exposed adults: A meta analysis. Journal of Consulting and Clinical Psychology, 74(4), 698-706.

Ozer, E. J., Best, S. R., Lipsey, T. L., \& Weiss, D. S. (2003). Predictors of posttraumatic stress disorder and symptoms in adults: A meta-analysis. Psychological Bulletin, $129(1), 52-73$

Palmblad, M., Tiplady, B. (2004). Electronic diaries and questionnaires: Designing user interfaces that are easy for all patients to use. Quality of Life Research, 13,11991207. 
Paranjape, A., \& Liebschutz, J. (2003). STaT: A three question screen for intimate partner violence. Journal of Women's Health, 12(3), 233-239.

Peeters, F., Nicolson, N. A., Berkhof, J., Delespaul, P., \& deVries, M. (2003). Effects of daily events on mood states in major depressive disorder. Journal of Abnormal Psychology, /12(2), 203-211.

Pitman, R. K., Gilbertson, M. W., Gurvits, T. V., May, F. S., Lasko, N. B., Metzger, L. J. et al. (2006). Clarifying the origin of biological abnormalities in PTSD through the study of identical twins discordant for combat exposure. In R. Yehuda (Ed.), Psychobiology of posttraumatic stress disorders: A decade of progress (pp. 242254). New York: Malden Blackwell Publishing.

Pitman, R. K., Orr, S. P., Forgue, D. F., de Jong, J. B., \& Claiborn, J. M. (1987). Psychophysiologic assessment of post-traumatic stress disorder imagery in Vietnam combat veterans. Archives of General Psychiatry, 47, 541-544. Plutchik, (1980). Emotion: A psychoevolutionary synthesis. New York: Harper \& Row. Preacher, K. J., Curran, P. J., \& Bauer, D. J. (2006). Computational tools for probing interactions in multiple linear regression, multilevel modeling, and latent curve analysis. Journal of Educational and Behavioral Statistics, 31(4), 437-448.

Price, J. L., Monson, C. M., Callahan, K., \& Rodriguez, B. F. (2006). The role of emotional functioning in military-related PTSD and its treatment. Anxiety Disorders, 20, 661-674.

Raudenbush, S. W., \& Bryck, A. S. (2002). Hierarchical linear models: Applications and data analysis methods ( $2^{\text {nd }}$ ed.). Thousand Oaks, CA: Sage. 
Reghler, C., LeBlanc, V., Jelley, R. B., Barath, I., \& Daciuk, J. (2007). Previous trauma exposure and PTSD symptoms as predictors of subjective and biological response to stress. The Canadian Journal of Psychiatry, 52(10), 675-683.

Resick, P. A., Nishith, P., Weaver, T. L., Astin, M. C., \& Feuer, C. A. (2002). A comparison of cognitive-processing therapy with prolonged exposure and a waiting condition for the treatment of chronic posttraumatic stress disorder in female rape victims. Journal of Consulting and Clinical Psychology, 70(4), 867879.

Reynolds, M., \& Tarrier, N. (1996). Monitoring of intrusive symptoms in post-traumatic stress disorder: A report of single case studies. British Journal of Medical Psychology, 69, 371-379.

Riggs, D. S., Dancu, C. V., Gershuny, B. S., Greenberg, D., \& Foa, E. B. (1992). Anger and post-traumatic stress disorder in female crime victims. Journal of Traumatic Stress, 5(4), 613-625.

Roemer, L., Litz, B. T., Orsillo, S. M., \& Wagner, A. W. (2001). A preliminary investigation of strategic withholding of emotions in PTSD. Journal of Traumatic Stress, 14(3), 149-156.

Schärer, L. O., Hartweg, V., Valerius, G., Graf, M., Hoern, M., Biedermann, C., et al. (2002). Life charts on a palmtop computer: first results of a feasibility study with an electronic diary for bipolar patients. Bipolar Disorders, 4(1), 107-108.

Schell, T. L., Marshall, G. N., \& Jaycox, L. H. (2004). All symptoms are not created equal: The prominent role of hyperarousal in the natural course of posttraumatic psychological distress. Journal of Abnormal Psychology, 113(2), 189-197. 
Schimmack, U., \& Diener, E. (1997). Affect intensity: Separating intensity and frequency in repeatedly measured affect. Journal of Personality and Social Psychology, $73(6), 1313-1329$.

Schnurr, P. P., \& Green, B. L. (2004). Understanding relationships among trauma, posttraumatic stress disorder, and health outcomes. In B. Schnurr and P. Green (Eds.), Trauma and health: Physical Health consequences of exposure to extreme stress (pp. 247-268). Washington, DC: American Psychological Association.

Schwartz, J. E., \& Stone, A. A. (1998). Strategies for analyzing ecological momentary assessment data. Health Psychology, 17(1), 6-16.

Simpson, T. L., Kivlahan, D. R., Bush, K. R., \& McFall, M. E. (2005). Telephone selfmonitoring among alcohol use disorder patients in early recovery: A randomized study of feasibility and measurement reactivity. Drug and Alcohol Dependence, $79,241-250$.

Solomon, R. C. (2000). The philosophy of emotions. In M. Lewis and J. Haviland-Jones (Eds.), Handbook of emotions $\left(2^{\text {nd }}\right.$ ed., pp. 3-15). New York: Guildford.

Southwick, S. M., Morgan, C. A., III, Nicolau, A. L., \& Charney, D. S. (1997). Consistency of memory for combat-related traumatic events in veterans of Operation Desert Storm. American Journal of Psychiatry, 154(2), 173-177.

Spybrook, J., Raudenbush, S., Liu, X., \& Congdon, R. (2006). Optimal design for longitudinal and multilevel research: Documentation for the "Optimal Design" software [Computer software manual]. Retrieved March 12, 2007, from http://sitemaker.umich.edu/group-based/optimal_design_software 
Srivastava, S., John, O. P., Gosling, S. D., \& Potter, J. (2003). Development of personality in early and middle adulthood: Set like plaster or persistent change? Journal of Personality and Social Psychology, 84, 1041-1053.

Stone, A. A. \& Litcher-Kelly (2006). Momentary capture of real-world data. In M. Eid and E. Diener (Eds.), Handbook of multimethod measurement in psychology (pp. 61-72).

Stone, A. A., Neale, J. M., \& Shiffman, S. (1993). Daily assessments of stress and coping and their association with mood. Annals of Behavioral Medicine, 15, 8-16.

Straus, M. A. Hamby, S. L., Boney-McCoy, S., \& Sugarman, D. B. (1996). The revised Conflict Tactics Scales (CTS2): Development and preliminary psychometric data. Journal of Family Issues, 17(3), 283-316.

Street, A. E., \& Arias, I. (2001). Psychological abuse and posttraumatic stress disorder in battered women: Examining the roles of shame and guilt. Violence and Victims, $16(1), 65-78$.

Street, A. E., Gibson, L. E., \& Holohan, D. R. (2005). Impact of childhood traumatic events, trauma-related guilt, and avoidant coping strategies on PTSD symptoms in female survivors of domestic violence. Journal of Traumatic Stress, 18(3), 245252.

Tabachnick, B.G., \& Fidell, L.S. (2007). Using multivariate statistics $\left(5^{\text {th }}\right.$ ed.). Boston: Pearson.

Tarrier, N., Sommerfield, C., Reynolds, M., \& Pilgrim, H. (1999). Symptom selfmonitoring in the treatment of posttraumatic stress disorder. Behavior Therapy, 30, 597-605. 
Tennen, H., Affleck, G., Armeli, S., \& Carney, M. A. (2000). A daily process approach to coping: Linking theory, research, and practice. American Psychologist, 55(6), 626-636.

Tennen, H., Affleck, G., \& Zatura, A. (2006). Depression history and coping with chronic pain: A daily process analysis. Health Psychology, 25 (3), 370-379.

Tjaden, P., \& Thoennes, N. (2000a, November). Full report of the prevalence, incidence, and consequences of violence against women (National Institute of Justice, Research Report, NCJ No. 183781). Washington, D.C.: U.S. Department of Justice.

Tjaden, P., \& Thoennes, N. (2000b). Prevalence and consequences of male-to-female and female-to male intimate partner violence as measured by the National Violence Against Women Survey. Violence Against Women, 6(2), 142-161.

van Eck, M., Nicolson, N. A., \& Berkhof, J. (1998). Effects of stressful daily events on mood states: Relationship to global perceived stress. Journal of Personality and Social Psychology, 75(6), 1572-1585.

Vansteedlandt, K., Van Mecchelen, I., \& Nezlak, J. B. (2005). The co-occurrence of emotions in daily life: A multilevel approach. Journal of Research in Personality, $39,325-335$.

Wagner, A. W., Roemer, L., Orsillo, S. M., \& Litz, B. T. (2003). Emotional experiencing in women with posttraumatic stress disorder: Congruence between facial expressivity and self-report. Journal of Traumatic Stress, 16(1), 67-75. 
Walker, E. A., Newman, E., Dobie, D. J., Ciechanowski, P., \& Katon, W. (2002). Validation of the PTSD checklist in an HMO sample of women. General Hospital Psychiatry. 24, 375-380.

Watson, D., Clark, L. A., \& Tellegen, A. (1984). Cross-cultural convergence in the structure of mood: A Japanese replication and a comparison with U.S. findings. Journal of Personality and Social Psychology, 47, 127-144.

Watson, D., Clark, L. A., \& Tellegen, A. (1988). Development and validation of brief measures of positive and negative affect: The PANAS scales. Journal of Personality and Social Psychology, 54(6), 1063-1070.

Watson, D., \& Walker, L. M. (1996). The long-term stability and predictive validity of trait measures of affect. Journal of Personality and Social Psychology, 70(3), $567-577$.

Weathers, F., Litz, B., Herman, D., Huska, J., \& Keane, T. (1993, October). The PTSD Checklist (PCL): Reliability, validity, and diagnostic utility. Paper presented at the annual meeting of the International Society for Traumatic Stress Studies, San Antonio, TX.

Woods, S. J. (2000). Prevalence and patterns of posttraumatic stress disorder in abused and postabused women. Issues in Mental Health Nursing, 21, 309-324.

Youngstrom, E. A., \& Green, K. W. (2003). Reliability generalization of self-report of emotions when using the Differential Emotions Scale. Educational and Psvchological Measurement, 63(2), 279-295. 
Zelenski, J. M., \& Larsen, R. J. (2000). The distribution of basic emotions in everyday life: A state and trait perspective from experience sampling data. Journal of Research in Personality, 34,178-197.

Zeman, L. L., Johnson, D., Arfken, C., Smith, T., \& Opoku, P. (2006). Lessons learned: Challenges implementing a personal digital assistant (PDA) to assess behavioral health in primary care. Families, Systems, and Health, 24(3), 286-298.

Zoellner, L. A., Goodwin, M. L., \& Foa, E. B. (2000). PTSD severity and health perceptions in female victims of sexual assault. Journal of Traumatic Stress, 13(4), 635-649. 
Table 1. Sample Characteristics

\begin{tabular}{|c|c|c|c|c|c|}
\hline & $\begin{array}{l}\text { Total sample } \\
(\mathrm{n}=38)\end{array}$ & $\begin{array}{l}\text { Emotion } \\
\text { subsample } \\
(\mathrm{n}=30)^{*}\end{array}$ & $\begin{array}{l}\text { Excluded based on } \\
\text { missing emotion } \\
\text { data }(\mathrm{n}=8)\end{array}$ & $\begin{array}{l}\text { PCL Daily } \\
\text { subsample } \\
(\mathrm{n}=27) * *\end{array}$ & $\begin{array}{l}\text { Excluded based on } \\
\text { daily PCL data } \\
(\mathrm{n}=11)\end{array}$ \\
\hline \multicolumn{6}{|l|}{ Sociodemographics } \\
\hline African American (\%) & 23.68 & 20.00 & 37.5 & 22.22 & 14.29 \\
\hline Age in years & $54.65(3.92)$ & $54.95(4.00)$ & $53.55(3.63)$ & $54.58(3.97)$ & $55.92(4.24)$ \\
\hline Divorced/separated (\%) & 81.58 & 83.33 & 75.00 & 81.48 & 85.71 \\
\hline $\begin{array}{l}\text { College degree or } \\
\text { graduate or professional } \\
\text { training }(\%)\end{array}$ & $54.06^{\mathrm{a}}$ & $48.27^{b}$ & 75.00 & 48.15 & $50.00^{\mathrm{d}}$ \\
\hline $\begin{array}{l}\text { Occupational status } \\
\text { (\% full- or part-time) }\end{array}$ & 55.26 & 56.67 & 50.00 & 59.26 & 57.14 \\
\hline $\begin{array}{l}\text { Income } \leq \$ 40,000.00 / \\
\text { year }(\%)\end{array}$ & $62.16^{a}$ & $58.62^{b}$ & 75.00 & $61.54^{c}$ & 71.43 \\
\hline \multicolumn{6}{|l|}{$\begin{array}{l}\text { Between-Subjects } \\
\text { Measures }\end{array}$} \\
\hline BFI neuroticism & $2.85(.69)$ & $2.82(.76)$ & $2.97(.27)$ & $2.85(.79)$ & $2.82(.39)$ \\
\hline PCL-past month & $33.68(10.42)$ & $33.87(11.00)$ & $33.00(8.57)$ & $32.78(11.00)$ & $38.14(10.68)$ \\
\hline
\end{tabular}

Note. Values presented are percentages $(\%)$ or means (standard deviations). Ns as noted with the following exceptions: ${ }^{a} \mathrm{n}=37 ;{ }^{\mathrm{b}} \mathrm{n}=$ $29 ;{ }^{\mathrm{c}} \mathrm{n}=26 ;{ }^{\mathrm{d}} \mathrm{n}=6$. * For testing hypotheses $1 \mathrm{a}-\mathrm{b}$ and $2 \mathrm{a}-\mathrm{b} ;{ }^{*} *$ For testing hypotheses $3 \mathrm{a}-\mathrm{b}$ 
Table 2. Means (SD) of Daily Experiences Averaged Across Women and Sampling Period

$\begin{array}{lll}\text { Frequency } & \text { Intensity } & \begin{array}{l}\text { Intraindividual } \\ \text { Variability }\end{array}\end{array}$

\begin{tabular}{lccc}
\hline Emotion & & & \\
Surprise & $.50(.36)$ & $3.05(.88)$ & $.61(.63)$ \\
Sadness & $.37(.29)$ & $2.94(1.05)$ & $.59(.53)$ \\
Anger & $.34(.25)$ & $2.76(.89)$ & $.66(.57)$ \\
Contempt & $.30(.38)$ & $3.34(1.08)$ & $.37(.55)$ \\
Fear & $.19(.31)$ & $2.81(.90)$ & $.27(.44)$ \\
Guilt & $.28(.37)$ & $2.74(1.01)$ & $.35(.50)$ \\
Shame & $.23(.36)$ & $2.44(.70)$ & $.16(.31)$ \\
Disgust & $.20(.28)$ & $3.00(1.02)$ & $.32(.52)$ \\
Joy & $.99(.04)$ & $3.58(.87)$ & $.54(.33)$ \\
Interest & $.97(.08)$ & $3.62(.97)$ & $.66(.39)$ \\
& & & \\
\hline PTSD Symptom Cluster &.--1 & & \\
Reexperiencing & --- & & $2.32(1.58)$ \\
Avoidance & --- & $9.35(3.71)$ & $1.76(1.23)$ \\
Hyperarousal & -- & & \\
\hline Stress & & $3.71(2.41)$ & $1.20(.99)$ \\
\hline
\end{tabular}


Table 3. Social Context of Daily Emotional Experiences

Non-social Social

\begin{tabular}{lll}
\hline Emotion & & \\
& & \\
Sadness & 51.64 & 48.36 \\
Anger & 47.95 & 52.06 \\
Contempt & 47.42 & 52.58 \\
Fear & 39.18 & 60.82 \\
Guilt & 47.55 & 52.45 \\
Shame & 39.27 & 60.73 \\
Disgust & 47.76 & 52.24 \\
Joy & 48.65 & 51.35 \\
Interest & 47.81 & 52.19 \\
& & \\
\hline
\end{tabular}

Note: Values presented are percentages representing frequency of occurrence across all rating occasions and women;

$N$ for each emotion $=1090$ rating occasions 
Table 4. Daily Emotion Modeled as a Function of Neuroticism and Past-month PTSD Symptom Severity

\begin{tabular}{|c|c|c|c|c|}
\hline \multirow[b]{2}{*}{ Fixed Effects } & \multicolumn{2}{|c|}{ Negative Emotion } & \multicolumn{2}{|c|}{ Positive Emotion } \\
\hline & Coefficient (se) & $t^{\dot{H}}$ & Coefficient (se) & $t^{\dot{*}}$ \\
\hline Overall mean emotion level $\left(\gamma_{00}\right)$ & $1.51(.08)$ & -- & $3.29(.09)$ & -- \\
\hline Neuroticism $\left(\gamma_{01}\right)$ & $.19(.12)$ & 1.53 & $-.36(.14)$ & $-2.62 * *$ \\
\hline $\operatorname{PTSD}\left(\gamma_{02}\right)$ & $.023(.009)$ & $2.70 *$ & $-.014(.01)$ & -1.43 \\
\hline Random Effects & Variance Component & $\chi^{2+1}$ & Variance Component & $x^{2 \ldots}$ \\
\hline Level-2 effect $\left(u_{0 j}\right)$ & .19 & $963.59 *$ & .24 & $541.91 *$ \\
\hline Level-1 effect $\left(r_{i j}\right)$ & .20 & & .45 & \\
\hline
\end{tabular}

Note: $\mathrm{se}=$ standard error

Tests the null hypothesis that the estimate for fixed effect $=0$; degrees of freedom $=27$

Tests the null hypothesis that residual variance after adding level-2 predictors $=0$; degrees of freedom $=27$

${ }^{*} p \leq .001,{ }^{*} * p=.01$ 
Table 5. Daily Emotion Modeled as a Function of Neuroticism, Past-month PTSD Symptom Severity, and Daily Stress

Model for Emotion

Means

Overall mean

emotion level $\left(\gamma_{00}\right)$

Neuroticism $\left(\gamma_{01}\right)$

$\operatorname{PTSD}\left(\gamma_{02}\right)$

$\begin{array}{llll}1.52(.099) & -- & 3.31(.11) & -- \\ .010(.17) & .59 & -.36(.18) & -2.01 \\ .032(.014) & 2.26 * * & -.025(.015) & -1.63\end{array}$

Model for Stress

$\underline{\text { Slope }}$

$\begin{array}{lllll}\text { Intercept }\left(\gamma_{10}\right) & .095(.029) & 3.25 * * * & -.063(.023) & -2.81 * * \\ \text { Neuroticism }\left(\gamma_{11}\right) & .017(.056) & .31 & -.038(.048) & -.78 \\ \text { PTSD }\left(\gamma_{12}\right) & .009(.004) & 2.15 * * & -.0017(.0034) & -.49\end{array}$

\begin{tabular}{lllll}
\hline Random Effects & $\begin{array}{l}\text { Variance } \\
\text { Component }\end{array}$ & $\chi^{2 \uparrow \dagger}$ & $\begin{array}{l}\text { Variance } \\
\text { Component }\end{array}$ & $\chi^{2 \uparrow}$ \\
\hline Emotion mean $\left(\mathrm{u}_{0 \mathrm{j}}\right)$ & .25 & $667.36^{* * *}$ & .28 & $291.89^{* * *}$ \\
$\begin{array}{l}\text { Emotion-Stress } \\
\text { Slope }\left(\mathrm{u}_{1 \mathrm{j}}\right)\end{array}$ & .014 & $120.36^{* * *}$ & .004 & $24.76^{*}$ \\
Level-1 effect $\left(\mathrm{r}_{\mathrm{ij}}\right)$ & .19 & & .39 & \\
\hline
\end{tabular}

Note: se $=$ standard error

Tests the null hypothesis that the estimate for fixed effect $=0$; degrees of freedom $=24$

Tests the null hypothesis that residual variance after adding level- 1 and level-2 predictors $=0 ;$ degrees of freedom $=24$

$* * * p \leq .005 . * * p \leq .05 * \mathrm{p}=.053$ 
Table 6. Daily Emotional Numbing Symptoms Modeled as a Function of Lagged Daily Reexperiencing Symptoms and Negative Emotion

Fixed Effects
Model for Daily Numbing Symptom Means

Overall mean daily numbing symptoms $\left(\gamma_{00}\right)$

$t^{\dot{\dagger}}$

Model for Lagged Slopes

Daily Reexperiencing Intercept $\left(\gamma_{10}\right)$

$.009(.074)$

.12

Daily Negative Emotion Intercept $\left(\gamma_{20}\right)$

$-.72(.72)$

$-.99$

Reexp x Negative Emotion Interaction $\left(\gamma_{30}\right)$

$-.47(.12)$

$-3.93 * *$

\begin{tabular}{lcc} 
Random Effects & Variance Component & $\chi^{2+}$ \\
\hline Numbing mean $\left(\mathrm{u}_{0 \mathrm{j}}\right)$ & 4.84 & $62.50^{* *}$ \\
Daily Reexperiencing Slope $\left(\mathrm{u}_{1 \mathrm{j}}\right)$ & .053 & $40.56^{* *}$ \\
Daily Negative Emotion Slope $\left(\mathrm{u}_{2 \mathrm{j}}\right)$ & 4.74 & $42.65^{* *}$ \\
Reexp x Negative Emotion Interaction Slope & .097 & $37.46^{*}$ \\
$\left(\mathrm{u}_{3 \mathrm{j}}\right)$ & & \\
Level-1 effect $\left(\mathrm{r}_{\mathrm{ij}}\right)$ & 1.94 & \\
& & \\
\hline
\end{tabular}

Note: $\mathrm{se}=$ standard error

${ }^{4}$ Tests the null hypothesis that the estimate for fixed effect $=0$; degrees of freedom $=26$

${ }^{1}$ Tests the null hypothesis that residual variance after adding level-1 and level-2 predictors $=0$; degrees of freedom $=26$

$* * p \leq .004, * p=.01$ 
Figure 1. Moderating effects of Past-month PTSD Symptom Severity on Associations Between Daily Stress and Negative Emotion

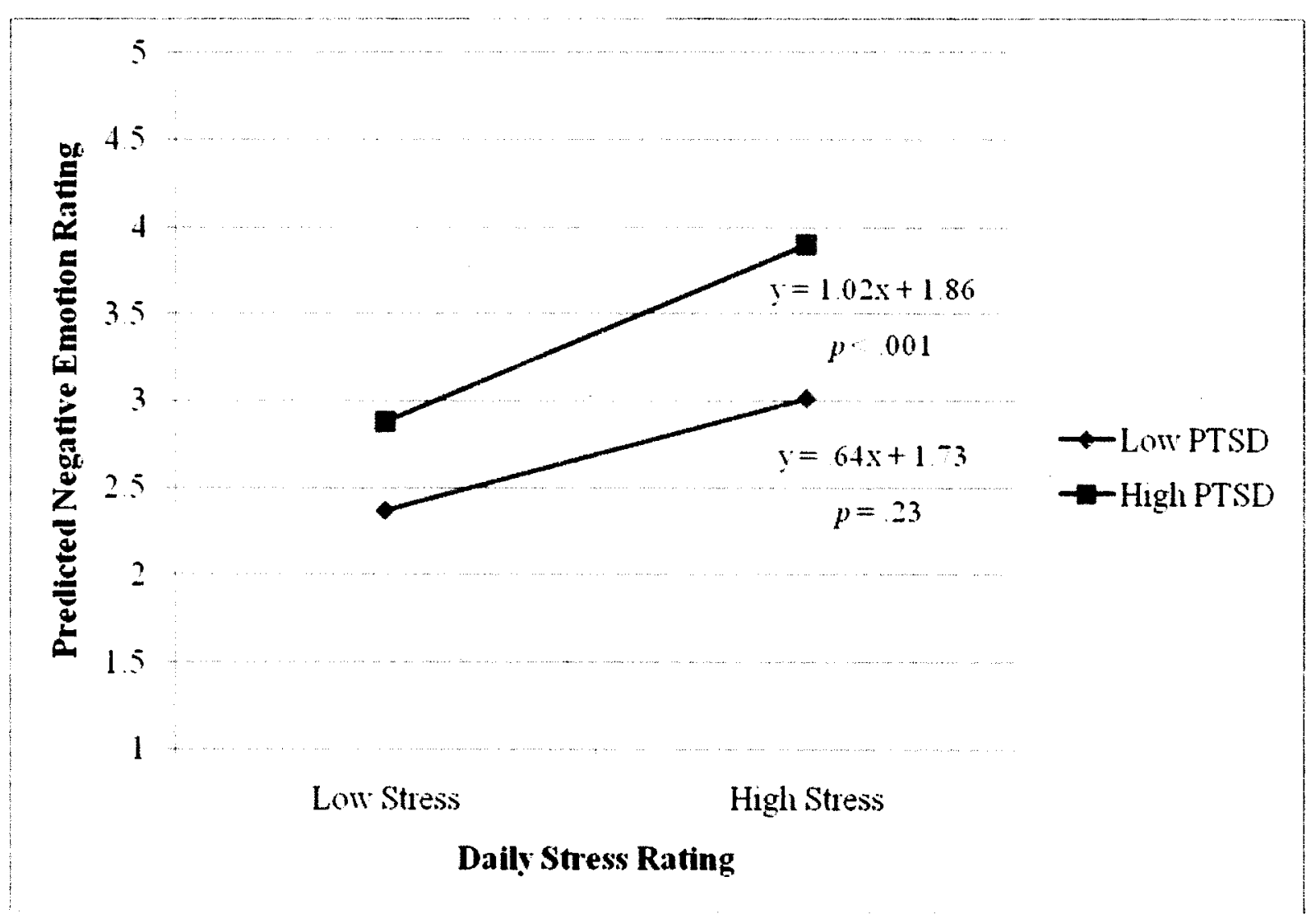


Figure 2. Interactive Effect of Previous Day Reexperiencing Symptoms and Previous Day Negative Emotion on Daily Emotional Numbing Symptoms

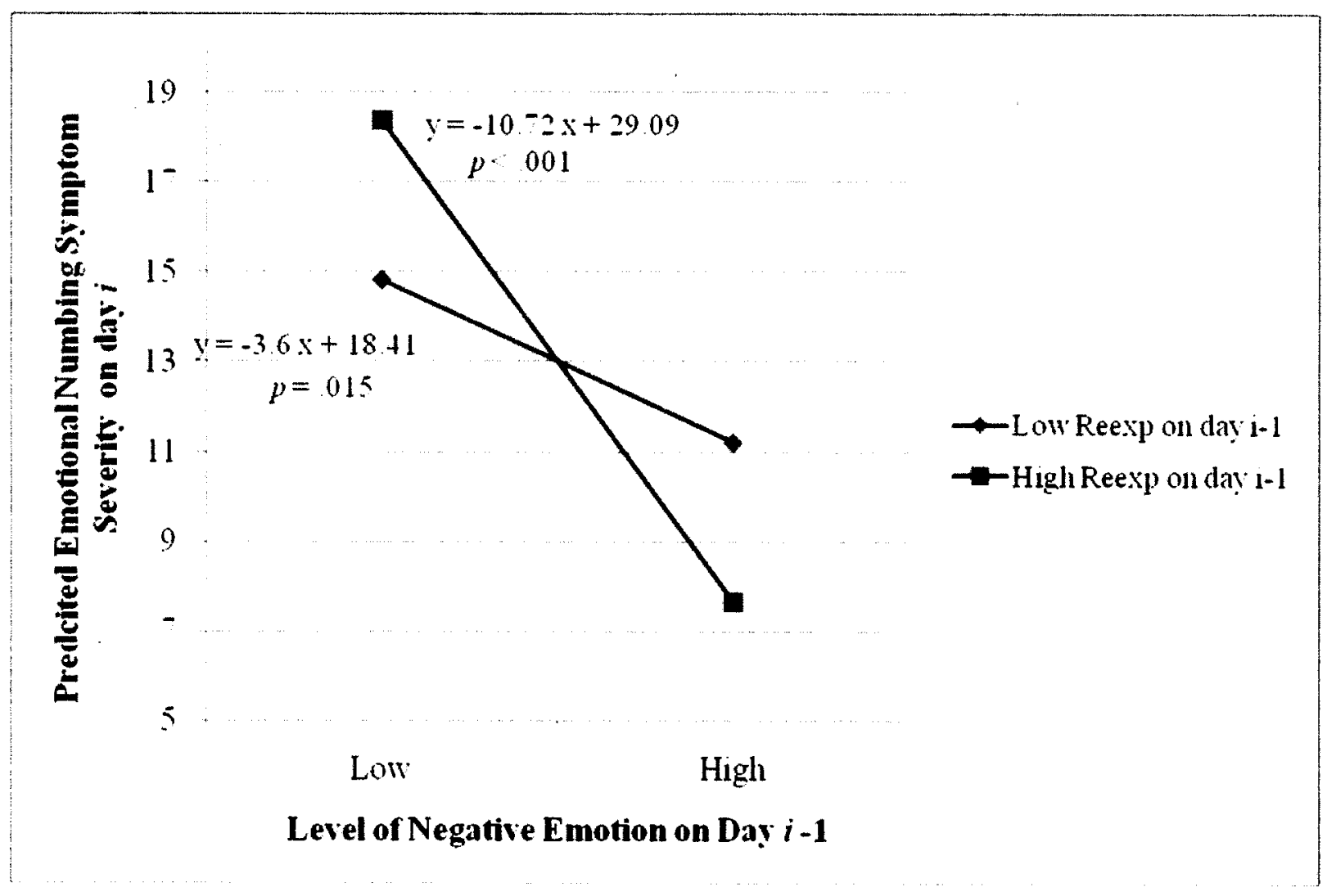


Figure 3. Proposed Model of Different Emotional Numbing Response Processes Based on Differentiated Emotional Responses to Intrusions

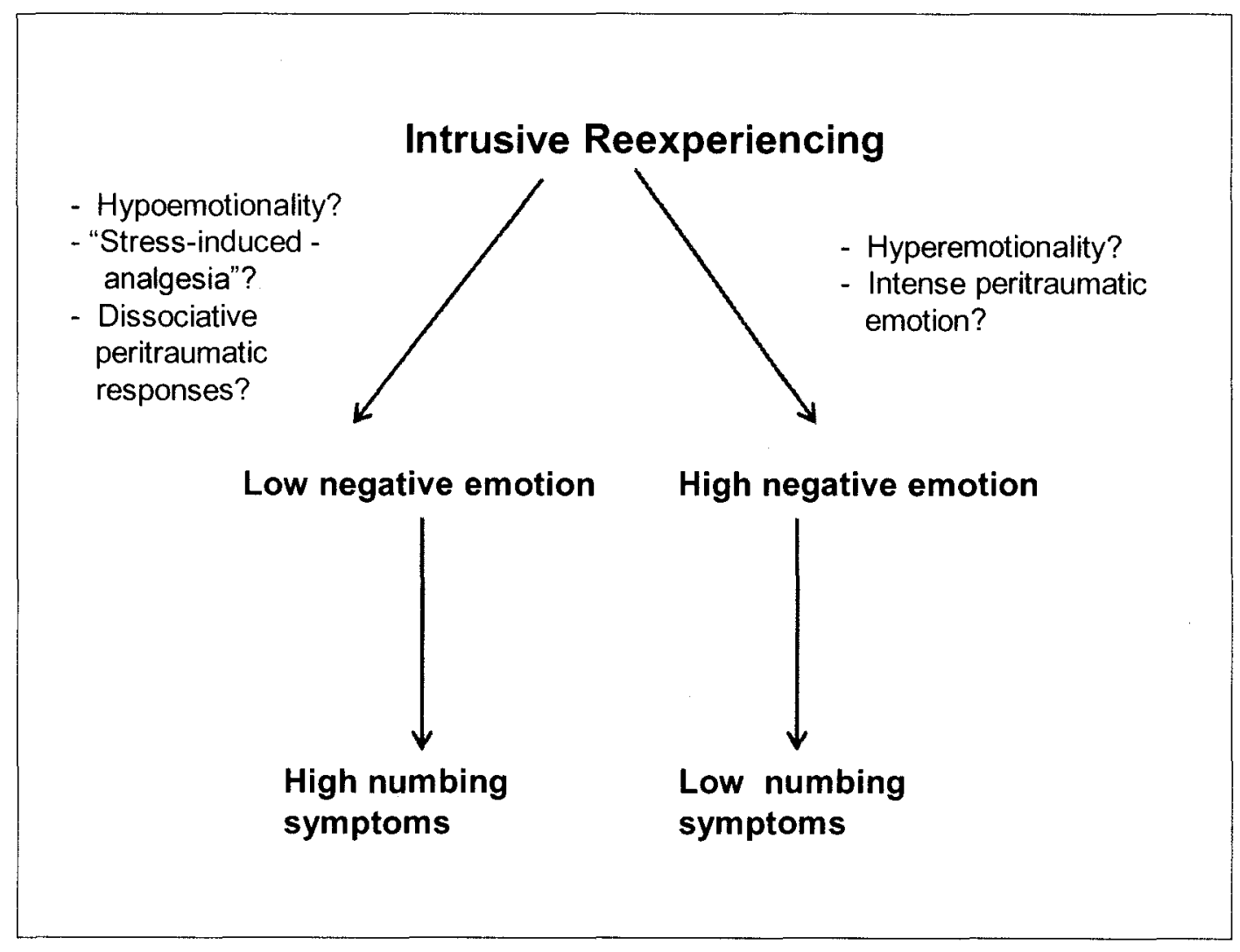




\section{CURRICULUM VITAE}

\section{REBECCA A. WEIGEL, M.A.}

\section{Contact Information:}

Department of Social and Behavioral Sciences

Century College

3300 Century Avenue North

White Bear Lake, Minnesota 55110

Phone: (651) 779-3459

Email: rebecca.weigel@century.edu

\section{EDUCATION:}

$2008-2009$

Minneapolis VA Medical Center, Minneapolis, Minnesota

Pre-doctoral Internship in Clinical Psychology

(APA-accredited program)

2003 - present

University of Louisville, Louisville, Kentucky

Department of Psychological and Brain Sciences

Doctoral Candidate, Clinical Psychology

$1998-2002 \quad$ Centre College, Danville, Kentucky

Bachelor of Science, Psychobiology, Magna cum laude

HONORS AND AWARDS:

2005

Meritorious Student Poster Award, Society of Behavioral Medicine

2005

Outstanding Teaching Assistant Award for Graduate Statistics

Department of Psychological and Brain Sciences, University of

Louisville

2002

Phi Beta Kappa, Centre College 


\section{TEACHING POSITIONS:}

2010 - present

2006

2006

2005

2005

2004

$2003-2004$

2000

2000

RESEARCH:

$2007-2008$

$2004-2007$

$2002-2003$
Psychology Instructor

Century College, White Bear Lake, Minnesota

Teaching Assistant, University of Louisville Abnormal Psychology

Teaching Assistant, University of Louisville Cognitive Assessment (Graduate level)

Teaching Assistant, University of Louisville Interviewing Skills (Graduate level)

Teaching Assistant, University of Louisville Advanced Statistics II (Graduate level)

Teaching Assistant, University of Louisville Advanced Statistics I (Graduate level)

Teaching Assistant, University of Louisville Introductory Psychology

Teaching Assistant, Centre College Experimental Methods in Psychobiology

Teaching Assistant, Centre College Introductory Psychology

Graduate Research Assistant, University of Louisville Health and Stress Research Lab

Project Title: Intimate partner violence and inflammatory processes

Source: NIH: National Institute on Aging

Graduate Research Assistant, University of Louisville Health and Stress Research Lab

Research Analyst, University of Louisville

Health and Stress Research Lab

Project Title: Social dominance, gender, and cardiovascular reactivity

Source: National Heart, Lung, and Blood Institute 
$2000-2002$

Institutional Review Board, Centre College

Faculty-appointed student member

\section{Grant Funding:}

Role: Co-investigator

Title: Posttraumatic Stress Disorder and Daily Emotions

Source: University of Louisville Arts \& Sciences Intramural Research Grant Dates: $9 / 1 / 07-6 / 30 / 08$

Total: $\$ 1,900$

\section{CLINICAL EXPERIENCE:}

$2008-2009$

$2006-2007$

$2005-2006$

$2005-2006$

$2003-2005$
Pre-doctoral Internship in Clinical Psychology

Minneapolis VA Medical Center

Major rotations: Geropsychiatry Team; Women's Clinic:

Posttraumatic Stress Recovery Team

Adjunctive rotations: Dialetical Behavior Therapy; Anxiety Interventions Clinic; Psychoanalytic Clinic; Assessment Clinic; Research Seminar

Clinical Psychology Practicum

Severe Mental Illness Treatment Team

University of Louisville

Nobel H. Kelly Psychological Services Center

Graduate Clinic Assistant

University of Louisville

Nobel H. Kelly Psychological Services Center

Clinical Psychology Practicum

HRSA Integrated Primary Care Treatment Team

University of Louisville School of Medicine

Ambulatory Internal Medicine Clinic

Clinical Psychology Practicum

Stress Disorders Treatment and Research Team

University of Louisville

Nobel H. Kelly Psychological Services Center 


\section{PUBLICATIONS:}

Harvison, K. W., Molfese, D. L., Woodruff-Borden, J., \& Weigel, R. A. (2009). Neonatal auditory evoked responses are related to perinatal maternal anxiety. Brain \& Cognition, 71, 369-374.

Newton, T.L, \& Weigel, R. A. (2007). Cardiovascular correlates of interpersonal mistreatment in healthy adults. Journal of Emotional Abuse, 7(2), 35-58.

Newton, T. L., Weigel, R. A., \& Watters, C. A. (2006). Socioemotional correlates of self- reported menstrual cycle irregularity: Replication and extension. Journal of Reproductive and Infant Psychology: 24(4), 304-313.

Newton, T. L., Watters, C. A., Philhower, C.P., \& Weigel, R. A. (2005). Cardiovascular reactivity during dyadic social interaction: The roles of gender and dominance. International Journal of Psychophysiology, 57, 219-228.

\section{CONFERENCE PRESENTATIONS:}

Weigel, R. A., \& Newton, T. L. (March, 2006). Physiological correlates of interpersonal mistreatment in women. Poster presented at the 2006 American Psychosomatic Society, Denver, CO, March.

Weigel, R. A., \& Newton, T. L. (April, 2005). Gender differences in associations between submission and salivary cortisol. Poster presented at the 2005 Society of Behavioral Medicine conference, Boston, MA.

Weigel, R. A., Watters, C. A., \& Newton, T. L. (April, 2005). Socioemotional characteristics are related to menstrual cycle irregularity. Poster presented at the 2005 Society of Behavioral Medicine conference, Boston, MA.

Watters, C. A., Weigel, R., \& Newton, T. L. (March, 2004). Social dominance, gender, and cardiovascular reactivity during dyadic social interaction. Poster presented at the Society of Behavioral Medicine, Baltimore, MD.

\section{COMMUNITY PRESENTATIONS:}

2008

Invited Speaker, "Stress and Your Health"

Dept. of the Army Fort Knox Equal Opportunity Office Brown

Bag Seminar, Fort Knox, KY

2008

Invited Speaker, "Women's Health and Stress" 
Federally Employed Women, Derby City Chapter meeting, Louisville, KY

2008

Invited Speaker, "Stress: Is it affecting your health?"

Ron Mazzoli Federal Building Educational Seminar, Louisville, KY

2005

Co-presenter. "Posttraumatic stress disorder" Catholic Charities, Louisville, KY

PROFESSIONAL AFFILIATIONS:

American Psychological Association, Graduate Student Affiliate

International Society for Traumatic Stress Studies, Student Member

\section{PROFESSIONAL SERVICE ACTIVITIES:}

Indiana University Southeast Psychology Fair, University of Louisville Psychology Department Clinical Psychology Program representative

2006

Clinical Curriculum Committee, Student member, University of Louisville

2006

Norton Hospital Celebrating Women Event Volunteer, University of Louisville Psychological Services Center representative

2005

Norton Hospital Celebrating Women Event Volunteer, mental health screenings

2005

Kentucky Psychological Association Heads-Up Kentucky Volunteer

References available upon request. 